\title{
THE RELATION OF THE PRINCIPLES OF LOGIC TO THE
}

\section{FOUNDATIONS OF GEOMETRY*}

BY

\section{JOSIAH ROYCE}

\section{INTRODUCTION.}

In the year 1886, in the Philosophical Transactions of the Royal Society, Mr. A. B. Kempe published A Memoir on the Theory of Mathematical Form, in which, amongst other matters, he discussed the fundamental conceptions both of symbolic logic and of geometry. The ideas there indicated were further developed, by Mr. Kempe, in an extended paper On the Relation between the Logical Theory of Classes and the Geometrical Theory of Points, in the Proceedings of the London Mathematical Society for 1890 . Despite the close attention that has since then been devoted to the study of the foundations of geometry, Mr. KeMPE's views have remained almost unnoticed. They concern, however, certain matters which recent research enables us to regard with increasing interest. I have been led, therefore, to attempt a restatement of KEMPE's logical-geometrical theory. The restatement has led me to conceptions which, although implied in those which Mr. Kempe emphasizes, present a number of aspects which I believe to be novel, so that a considerable part of the present research follows a path of its own. My introductory words will indicate the nature of KEMPE's contribution to the problem of the foundations of geometry, the kind of task which his work has set before me, and my own main interest in preparing this paper.

The fundamental ordinal relation of geometry is the relation which can be, at pleasure, described as the triadic relation "between," or as an asymmetrical, transitive dyadic relation, such as "before," or " antecedent to," or " sequent to." Essentially the same relation is at the root of all serial order, and on this basis the logic of such order has lately been elaborately discussed by Mr. BERTRAND Russell, in his Principles of Mathematics.

The axioms of geometry, as Dr. VeBLEN has stated them (Transactions of the American Mathematical Society, July, 1904), consist (1) of

* Presented to the Society April 29, 1905. Received for publication May 5, 1905. 
assertions characterizing the "between" relation, and duly restricting the application of this relation so far as the "lines" of geometry are concerned, and (2) of existential propositions defining certain entities that shall possess the relation. A similar prominence of asymmetrical transitive relations appears in Dr. Huntington's various Sets of Postulates for numbers, groups, etc. (Ibid., January and April, 1905).

The algebra of logic may be viewed (as Dr. Huntington, following Mr. Peirce and Schroeder, has lately afresh shown in detail), as depending upon the relation of inclusion or subsumption, sometimes symbolized by $<$. This relation is dyadic and transitive, and may be either symmetrical or unsymmetrical. Upon the basis of this one relation we can define the various operations of formal logic, such as logical multiplication and addition. If the relation $<$ is in a given instance symmetrical, it ensures what is commonly viewed as the " uniqueness" of an entity. That is: $a<a$; and if $a<b$, while $b<a$, than $b=a$ (see Dr. Huntington's paper of July, 1904, in these Transactions, for a fuller statement of the various results of these considerations). The relation $a<b$, in so far as it obtains between non-equivalent elements, may serve to define linear series: $a<b<c<d$, etc.; where $a<c$, and $a<d$. In such a series $c$ may obviously be said to lie "between" $b$ and $d$, and the analogy to the geometrical relation "between" is in so far plain. "Dense," and in fact, continuous linear series of the subsumption type can be conceived after the analogy of point series. But on the other hand, a system of logical classes differs, with respect to linear relations, from a system of points on a line in two very notable ways :-

(1) If $a<b<d$, and if it is also true that $a<c<d$, any one of the three relations $b<c, c<b, c=b$, is indeed possible; but, in case of the logical entities, it is also possible that $b$ and $c$ are such that no one of these relations actually holds between these two. Thus, Siberia is included within the Russian Empire, which itself may be viewed as included within the "Eurasian" continent. And Siberia is also included in Asia, which may also be regarded as included within the "Eurasian" continent. These subsumptions are transitive, and in so far linear in their type. Yet the Russian Empire and Asia do not form a pair possessing the relation $<$, read in either sense.

(2) If $a<b<c<d$, and if, also, $i \prec b<c<j$, the relations of $i$ and $a$, of $j$ and $d$ are similarly left indeterminate. These relations need not be directly expressible in terms of $<$ at all. That is, nothing in the logical relations forbids linear series (whether dense, or continuous or not) to have two or more "points," i. e., elements, in common, while any number of the other elements of the series remain entirely distinct. The logical lines, as Mr. KeMPE observes, may intersect any number of times.

For this very reason, however, the system of logical entities may be viewed 
simply as much more general and inclusive than the system of the points of space. And thus it becomes possible to regard a given space-form as a selection from amongst the entities present in a system that exemplifies the logical relation $<$. That is: One may view the points of a space as a select set of logical elements, chosen, for instance, from a given "universe of discourse." This thought, whose possible fruitfulness for the logical development of the foundations of geometry I regard as highly notable, is the essential thought at the basis of Mr. KEMPE's paper of 1890 , cited at the outset of this introduction.

The reason why such a thought seems promising is this: The relations amongst logical entities are, in any case, the most fundamental relations that we know. Experience shows us in the outer world those ordinal space-relations which geometry generalizes in the concept of "between." But our own thinking processes show us the meaning of the logical relation $<$. The latter relation, then, is more suited to be the basis for a theory of the logic of an exact science, in case we can only so define and restrict its application that our ideal geometrical relations can come to be viewed as special instances of those forms which we can develop by the use of pure logic.

Mr. KEMPE's procedure, in the paper of 1890, is, in bare outline, as follows: $\mathrm{He}$ sets out, not by assuming the ordinary algebra of logic, but by defining, through postulates, a purely abstract set of entities called by him the "basesystem," and a relation which may be viewed as a generalized "between." The latter is the relation which, in its most general form, is characteristic of what KEMPE himself calls, later in his paper, "flat collections" of any number the elements of the "base-system." But the relation first appears as a triadic relation, and is so characterized in the postulates. KeMPE uses the notation : $a c \cdot b$, to mean the assertion: " $a, b$, and $c$, form a 'linear triad,' with $b$ between $a$ and $c . "$ So far the expressions used resemble those for Dr. Veblen's generalized relation " in the order $a b c . "$ But KEMPE's linear triad has these fundamental properties: (1) "If $a b \cdot c$, and $a=b$, then $c=a=b . "$ (2) "If $a=b$, then $a c \cdot b$ and $b c \cdot a$, whatever entity of the system $c$ may be." ${ }^{*}$ In other words, KEMPE permits the "between" relation to hold where the related elements are, for all the purposes of the operations of the system, identical; and then he defines the distinctness of elements by means of a restriction of the relations that are permitted to hold in triads of distinct elements. The result is that the "between " relation becomes Dr. VeBles's " in the order," whenever the elements are all distinct.

The other properties of the "between" relation which are in question for

* I vary a little the order of Mr. KeMPE's statement of his principles. The relation = is defined by Mr. KeMPE only in a very highly' abstract form which I need not here attempt to discuss. Geometrically interpreted, if this relation holds between points, these become identical points. 
Mr. KEMPE, are obtained by him through assuming two forms of triadic "transversal" propositions as fundamental postulates, viz. : *

I. If two linear triads, $a p \cdot b$ and $c p \cdot d$, exist, such that (as indicated by the notation), $b$ is between $a$ and $p$, and $d$ is between $c$ and $p$, then there exists an entity, $q$, which lies, in a linear triad, between $a$ and $d$, and, in another linear triad, between $b$ and $c$.

II. If, in the linear triads $a b \cdot p$ and $c p \cdot d$ (as indicated by the notation), $p$ lies between $a$ and $b$, and $d$ between $c$ and $p$, then $q$ exists such that $q$ lies, in a linear triad, so that $d$ is between $a$ and $q$, while, in another linear triad, $q$ is between $b$ and $c$.

If one interprets these assertions as relating to points in space, they become assertions obviously relating, respectively, to the diagrams following. But, as

I.

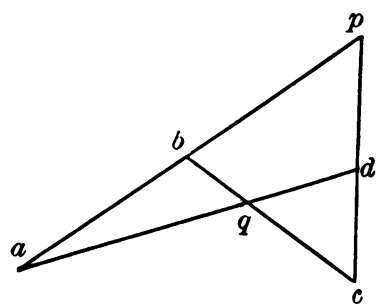

II.

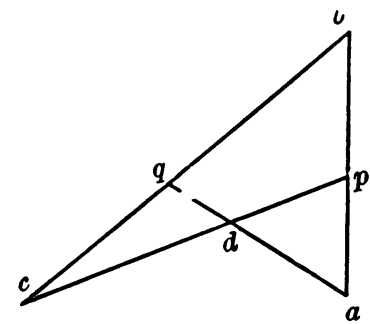

they are stated at the outset of Mr. KEMPE's paper, these principles have no specification beyond what the general properties of the linear triad, as just defined, predetermine.

One other existential proposition Mr. KEMPE uses as his fifth fundamental principle. This is simply the proposition that any entity belongs to the basesystem whose presence there is not inconsistent with the four other principles, - a proposition which of course formally renders the two existential principles, here numbered I and II, superfluous; and which leaves the account of the "base system" inevitably somewhat unsatisfactory.

Mr. KEMPE now proceeds upon this basis, to show, by a decidedly original, although necessarily intricate procedure, that the elements of the base system, as thus defined, possess the properties and relations of a system of logical classes, or of other entities subject to the algebra of logic. In other words, he develops the entire algebra of logic, including the definitions and properties of the operations of logical multiplication and logical addition, without any other assumptions than those simple properties of the "between" relation which have just been stated. The proofs given are such as to apply to any finite number of the elements. Mr. Kempe leaves, however, some doubt as to infinite collections.

* I vary slightly Mr. Kempe's mode of enunciating these existential propositions at the ontset of his paper. 
Highly instructive observations are incidental to this development. The system of logical entities appears as possessing a thoroughly symmetricai structure. The "zero."-element and the "universe"-element have no essential distinction from any other similarly related pair of "obverse" elements. Negatives, in general appear as "obverses," because of the symmetrical contrast of their respective relations to the remainder of the system. All the fundamental relations of logic appear as triadic rather than as dyadic. But upon this triadic basis, polyadic relations also develop-the relations of KEMPE's "Flat-collections." These collections, thus named by reason of their resemblance to the various possible configurations of points in an $n$-dimensional space- " on a line," "on a plane," "in a three dimensional space," etc. - are Mr. KeMPE's means of relating the purely logical to the geometrical entities.

The junction of his principles with the regular algebra of logic once completed, although leaving certain doubts as to the application of his proof to infinite sets, KEMPE proceeds to the geometrical application. By (1) selecting certain "linear sets" of the elements of the base system; and (2) selecting from these sets those which conform to a new principle (here for the first time introduced into the essay), namely, to the principle that any two of the elements of a selected linear set shall determine the whole linear set to be selected, KEMPE is in possession of a system of foundations for a geometry of a " flat space" of $n$ dimensions. The further development of such a geometry is indeed merely sketched in the paper in question. But since the "triangle-transversal" axiom is provided for by the initial principles of the system, and since, by the selection of the linear sets of elements, the ordinary properties of the geometrical " between," and the axiom as to the determination of a line by any two of its points are now also secure, KEMPE's result, although only indicated in his text, is in the main clear. A space of $n$ dimensions is a select class or set of elements which themselves are entities in a logical "field." The selection of the entities of a given space is arbitrary; and so the space-forms whose entities are selected may be varied in any way whatever which is consistent with the triangletransversal-axiom, and with the properties of the generalized between-relation. The problem of the continuity of the geometric sets is only very generally treated, and is not solved.

The wide outlook thus suggested into the theory of space-forms certainly deserves to be better considered than KEMPE's treatment of the subject has so far been considered by mathematicians. For me, however, as a student of philosophy, a still further interest attaches to those results which $I$ have thus suggested, an interest which my mathematical colleagues may also share.

The problem of the foundations of geometry is only a part of that general problem regarding the fundamental concepts of the exact sciences which is now so widely studied. KEMPE's research suggests that, since metrical relations, and 
therefore (as KeMPE himself briefly indicated in his Theory of Mathematical Form, § 309), the whole algebra of ordinary quantity, can be reduced, in any system of three or more dimensions, to a series of propositions based upon purely ordinal relations, - the entire system of the relationships of the exact sciences stands in a much closer connection with the simple principles of symbolic logic than has thus far been generally recognized.

Mr. Bertrand Russell, using very different methods, insists, indeed, in general, upon the closeness of such a connection. But the distinction between the "logic of relations," and the older "logic of classes," and of "propositions," a distinction which Mr. Bertrand Russell in his Principles of Mathematics regards as something quite fundamental, seems to me to become, in the light of KEMPE's research, a distinction probably quite superficial. Hence to my mind, Mr. Kempe's theory goes far deeper than Mr. Russell's. Give us a system of entities of the types of logical classes, and we shall find that their relations (all statable in terms of KEMPE's "between"), are already (quite apart from a separate "logic of relations"), certainly as rich as the totality of the relations studied in geometry, and are, for reasons upon which KEMPE has dwelt, probably as rich as the totality of the relations known to the exact sciences, at least so far as the latter have yet been developed. The bare prospect of such a result deserves a careful consideration, in case one takes interest in the unification of the categories of science. KEMPE's theory promises such an unification.

The present memoir proposes to contribute towards a more precise statement of the theory thus outlined. At the basis of my own discussion, I place, however not KEMPE's " between " relation, but another fundamental relation of symbolic logic which has the interest of being absolutely symmetrical, while, when it obtains amongst $n$ entities, it permits (upon the basis of certain simple existential propositions), the definition of the properties of Kempe's "flat collections," and so the definition of asymmetrical relations of a very high degree of complexity. This change of starting point is the prime novelty of the present discussion, as contrasted with KEMPE's.

The contrast between symmetrical and unsymmetrical relations seems, to the ordinary view, absolute. Mr. Russell, in his late volume, so treats it. Geometry, and the ordinary algebra of quantity (as these subjects are usually treated), seem to depend on regarding the distinction as quite fundamental. In symbolic logic, however, as Mrs. LADD-FrankLin long ago pointed out (in her paper on the algebra of logic in the volume called "Studies in Logic by members of the Johns Hopkins University," Boston, 1883), a "symmetrical copula," namely that of "inconsistency," or of "opposition," can be made to accomplish all the work of the ordinary unsymmetrical copula $<$. In other words, if I have otherwise defined the meaning of "not," the statement " $x$ is inconsistent with not- $y$," means the same as " $x$ implies $y . "$ The copula in the former case is 
symmetrical, in the latter unsymmetrical. The former expression makes explicit the "relative product" (as it is called by PeIRCe and Russell) of two symmetrical relations (viz., "opposes" and "not"). This "relative product" is, itself, indeed an unsymmetrical relation. But the constituents of this product are symmetrical. This already suggests how asymmetry may be definable in terms of symmetry.

Using as my suggestion some brief observations of KEMPE (in $\S \S 75-82$, of his paper in the Proceedings of the London Mathematical Society), I have therefore chosen to define, by postulates, at the outset of my discussion, a symmetrical relation which I may call "the $O$-relation." This relation is essentially polyadic, and applies at once to any number of terms greater than one. In logical terms, this is the relation in which (if we were talking of the possible chances open to one who had to decide upon a course of action) any set of exhaustive but, in their entirety, inconsistent choices would

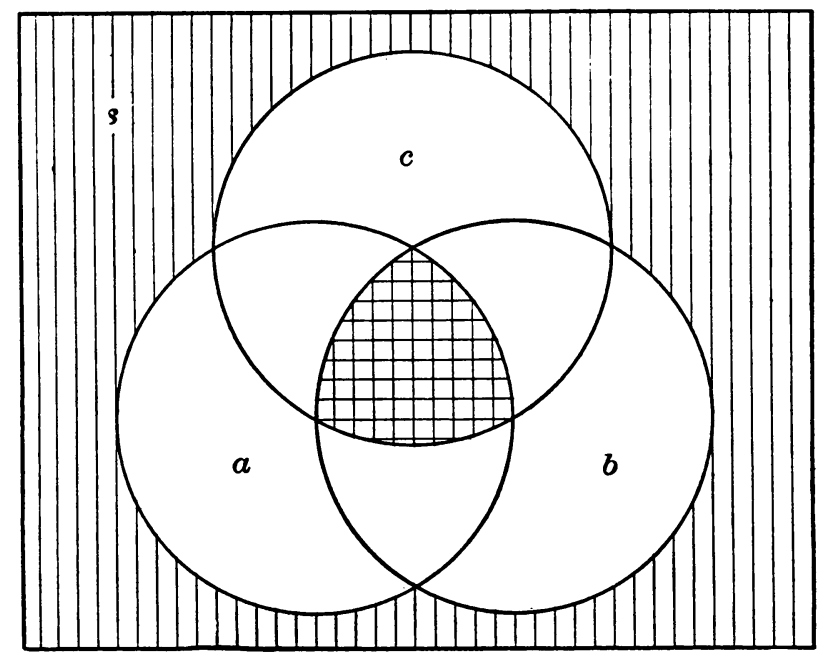

stand to one another. It is also the relation in which the members of any set of areas stand to one another when there is no area (except the "zero"-area) which is common to all the areas of the set at once, while together these areas exhaust some larger surface (which therefore resembles, in its relation to them, a logical "universe"). Thus if, in the annexed diagram, the surface $s$ contains three circles, $a, b$, and $c$, and if we then agree to disregard, or to view as stricken out or destroyed, the here shaded portions of the diagram, the circles $a, b$, and $c$ have then only the stricken-out or "zero" area in common, while together they exhaust what we thus permit to remain of the surface s. In consequence, $a, b$, and $c$ here form what KEMPE calls "an obverse collection," and what I call, in this paper, an "O-collection." 
If $t w o$ objects stand in the $O$-relation each to the other, then these two are related as a pair of "negative" or contradictory classes, or statements, are related to one another. But any number or multitude of objects, in case such are otherwise permitted to exist in a given system, may stand, not in pairs, but as a whole collection, in this relation, and will then constitute what $I$ call an "O-collection." The name that I give to the relation is derived from the close analogy of such collections, even when they contain more than two elements, to KEMPE's pairs of "obverse elements." But I do not myself wish to call the O-collections, with KEMPE "obverse collections," because, as will be seen, I find it convenient to use an expression in my text (where I speak of "mutually obverse" collections), in a way that would make such usage confusing. Hence I read the expression " $O$-collection," simply as written.

In my text the $O$-relation is entirely freed from dependence upon all such examples as the ones just used, and is defined solely by postulates, and is to be taken solely as it is there defined. The fact however that it is, in its relational properties, identical with the "yes-no" relation - the earliest exact relation defined by the human mind - is, in this introduction, important. For what I am in the end to show is that all the serial and other ordinal relations known to logic and to geometry, and all the operations known to both, so far as they are pure exact sciences, are ultimately reducible to assertions that certain entities do, while certain entities do not stand to one another in the perfectly symmetrical O-relation.

My procedure differs from KEMPE's, not only in making this wholly symmetrical relation, instead of KEMPE's "between," fundamental, but also in the existential principles which I assume. KEMPE's "transversal" axioms form with me a theorem, proved late in the discussion. My own existential principles have to be wide enough to provide for the "continuity" of the system, or, rather, for its inclusion of infinitely numerous continuous systems, and definite enough to make the system of the entities to which the logical calculus is applicable a determinate manifold, inclusive of the points of a space of $n$-dimensions. The usual treatment of the algebra of logic provides only for arbitrarily determined sets of $2^{n}$ or of $2^{2 n}$ entities in a given logical system. KEMPE calls any such selected set a "full set." KenPE's further postulate, however, calling for "all entities" consistent with the formal laws, is itself indefinite. In seeking adequate postulates $I$ have been led to two observations which, although in themselves fairly obvious, seem to me to be new, viz., (1) a relation is here shown between the existence of logical sums and products and the general theory of limits and of continuity; and (2) a general definition of the pairs of elements which I have called "conjugate resultants"*

* This concept of " oonjugate resultants" is generalized from KEMPE's own generalization of his "unsymmetrical resultants" in $\S 28$ of the essay of 1890 . My use of the concept differs in many ways from his. 
is made centrally important. The algebra of logic, so far as I know, has not hitherto been brought into definite relations with the problem of the continuum. This is one of the things that $I$ here accomplish. This undertaking involves proving all the principles of logic so as to make them applicable to infinite sets of entities at once. This also I have here done.

KEMPE's " linear triad" of elements is represented, in any logical system of classes, by the classes, or areas $a, b$, and $c$, which stand in the relation which is represented in the adjoining diagram by the closed figures so lettered. Any area $c$ which includes the common part of $a$ and $b$, and which is included within their logical sum, is, in KEMPE's phrase, such that " $c$ is between $a$ and $b . "$ I hereafter symbolize this relation, in my own way, as $F(c \mid a b)$. The relation in question is called by me the $F$-relation, because it is that characteristic of

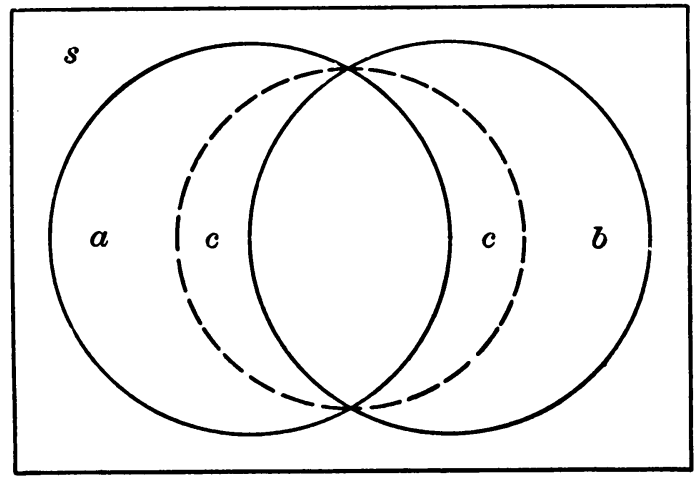

KEMPE's "flat collections." The $F$-relation, so long as "obverses" or " negatives" exist, follows immediately from, and is equivalent to, an $O$-relation. For, in the diagram if $s$ is the total surface in which $a, b$, and $c$ are included, then when " $c$ is between $a$ and $b$," " $a, b$, and $\bar{c}(\bar{c}$ being the obverse of $c$ ) constitute an $O$-collection," or "are in the $O$-relation."

The outcome of our discussion will show that, while logical relations can be indifferently stated as $O$-relations, or stated as $F$-relations, or (when once addition, multiplication, and negation have been defined) can be stated in terms of equivalence, the $F$-relations are the only natural means of expressing the geometrical ordinal relations. This difference, however, between the logical and the geometrical entities, is due to the simple fact that (as KEMPE points out), when geometrical sets are considered, the obverses of the elements of any set are excluded from that set, so that the obverses may be viewed as ideal elements of the geometry in question. In fundamental meaning all these relations spring from a common root.

If " $m$ is between $b$ and $c$," I sometimes call $m$ "mediator" or again, on occasion, "resultant" of $b$ and $c$. I extend the term "resultant" to include 
the case where a single element stands in an $F$-relation to any collection of elements.

The axioms or principles assumed at the outset of my discussion, in $\$ 19$, are verifiable for a collection of areas all of which are included within a given area, if the $O$-relation is interpreted as, for the sake of illustration, $I$ have just done. The consistency of these axioms is thus secured from the start. For the verification of "Principle VI" of my set of principles, see $\S 118$ of my text. For KEMPE's term " base-system," I substitute " the system $\Sigma$."

The considerable length of the discussion may be justified by the importance, (1) Of a development of the principles of logic solely in terms of a symmetrical polyadic relation; and (2) Of the need of supplementing KEMPE's results by a theory of the continuity of the "base-system."

\section{THE SYSTEM $\Sigma$, AND THE O-COLLECTIONS.}

\section{Chapter I. Definitions and Principles.}

1. The system $\Sigma$, whose structure we are to consider in what follows, consists of certain "elements," which we shall regard, in the present discussion, as simple and homogeneous. As symbols for these elements, we shall employ the small letters of the alphabet: $a, b, \cdots, i, j, \cdots, x, y$, etc. In many cases, for the sake of distinguishing one element of a set from others, we shall need subscript marks; and for these too we shall nearly always employ small letters, or if that be convenient, numbers, thus: $a_{k}, b_{i}, \cdots, x_{1}, x_{2}, p_{v}$, etc. It is to be noted however, that, unless the contrary is especially indicated, these subscripts are merely convenient distinguishing marks; so that the numbers when used as subscripts will, in general, not possess any ordinal meaning, but will be used merely as tags. The few exceptions to this rule will explain themselves.

2. The elements thus symbolized may be viewed either singly, or in their collections. A collection of elements will usually be in question, in what follows, as a sort of complex or secondary unit. We shall apply predicates to collections when they are viewed as such complex units, shall compare collections, combine them into larger collections, make partitions of them into the partial collections of which larger collections are composed, classify collections, etc. A collection may consist of a single element of $\Sigma$, and is then called a monad, or a monad-collection. A collection of two elements is called a pair, of three a triad, of four a tetrad, of $n$ elements an $n$-ad. But a collection may consist of an infinite multitude of elements. And, in fact, whenever our statements, and whenever the conditions imposed in the course of a given investigation, do not set definite limits to the multitude of the elements that belong to such collections as are at any time in question, it is always to be understood that the collections 
of which we then speak are actually permitted to consist not only of any finite number, but of any multitude whatever of elements.

3. Collections in which a single element, such as $b$, or $x$, is viewed as occurring repeatedly, are to be regarded as permissible objects for our consideration ; and if we define the number or the multitude of elements in such a collection, or if, for any other reason, we have to treat all the various elements of a given collection in various ways, then, for the purposes of the count, or of the other individual treatment of the elements of any collection, the various repetitions of a given element are to be treated as, in so far, distinct members of the collection in question. Empty, or " zero" collections will not be considered in the present discussion.

4. In order to symbolize a collection without indicating, by the mere symbol, any assertion except the assertion that the collection consists of certain elements, we shall write the symbols for the elements in question, separated by commas, and in a parenthesis. Thus the symbol $(a, b)$ denotes "the pair which consists of the elements $a$ and $b$." The symbol $(x, x)$ denotes " the pair consisting of $x$ and of $x$ repeated." The symbol $(x, x, y, y, r)$, denotes " the collection consisting of $x$ and of $x$ repeated, and of $y$ and of $y$ repeated, and of $r$." The symbol $\left(x_{1}, x_{2}, \cdots, x_{r}, \cdots\right)$ denotes " the collection consisting of $x_{1}$, of $x_{2}$, and of an unspecified multitude of other elements, each of which is symbolized by $x$ written with some subscript." In such a case, if no restriction of the multitude of the elements is stated, this multitude of the elements of the collection need not be limited to that of the whole numbers; and the use of whole numbers as subscripts is then of no special significance, beyond that of the convenience of such subscript-symbols. Other subscript-symbols would be equally possible, and may, upon occasion, be used.

5. In many cases, we shall need to symbolize a collection without at the moment designating any of the single elements of which it consists. In such case we shall use Greek letters, $\alpha, \beta, \gamma, \delta$, etc. (and, in a few cases, the capital Greek letters also), as symbols for entire collections. Thus, the symbol $\alpha$ means "the collection designated as $\alpha$, consisting of elements which are not hereby further specified." In such a case, the collection $\alpha$ may be, in fact, a perfectly determinate collection, and the symbol $\alpha$ will then be merely a convenient abbreviation. In other cases, $\alpha$ may stand for an unspecified instance of some class of collections; the members of the collection at any time in question being left, by the conditions of the discussion, to be otherwise determined. A collection $\alpha$ or $\beta$ or $\delta$ may be unrestricted as to the multitude of its elements, or may be a monad, a pair, a triad, etc., according as the conditions of a given statement permit or determine.

6. A frequent operation, in our discussion, will be the adjunction of elements to an already given collection, or to elements already under consideration. The 
symbol $(\alpha, x)$ will mean "the collection consisting of the collection $\alpha$ of elements together with the element $x$, adjoined thereto." The symbol $(\alpha, \beta, \gamma)$ will mean "the collection formed by adjoining to the collection $\alpha$ all the elements of the collections $\beta$ and $\gamma . "$ Greek letters, as symbols for whole collections, and small letters $a, x, m$, etc., as symbols for elements, may thus be combined in the same expression in order to indicate what adjunctions are at any time in question.

7. When we speak simply of " a collection" of the elements of $\Sigma$, without further specification of the character of this collection, the order in which the elements of the collection are named, or otherwise indicated, or in which they stand in the collection, is wholly indifferent. A collection, in such a general case, is determined wholly by the fact that certain elements do, while certain elements do not belong to it. The arrangement of the elements within the collection will concern us only in case the definition of a given type of collections, or the conditions of a given problem, expressly require us to take note of such arrangement.

8. Fundamental, in our discussion of the properties of the system $\Sigma$, is a classification of the collections of elements into those which are, and those which are not what we shall call "O-collections." The $O$-collections form a class of collections whose fundamental properties we define by the laws hereafter stated. The symbol $O(x y z \cdots)$ is to be read as the statement: "The elements $x, y, z$, etc., taken together, constitute an O-collection." The symbol $O(\alpha)$ is to be read as the statement: "The collection $\alpha$ is an $O$-collection." The symbol $O\left(\beta_{\gamma}\right)$ is to be read as the assertion that "The total collection formed out of the collections $\beta$ and $\gamma$ is an $O$-collection." At pleasure we shall also use the abbreviated form of expression: "the collection $O(x y z \cdots)$," meaning "the collection such that the assertion $O(x y z \cdots)$ is true." The symbol $O(\alpha x)$ means that " the collection formed by adjoining the element $x$ to the collection $\alpha$ is an $O$-collection." The symbols $O(\alpha \beta \gamma \delta), O(x y \alpha p q \beta j r \delta)$, etc., are to be read so as to assert that whatever total collection of elements and collections is indicated by the letters enclosed in the parentheses, is an $O$-collection.

9. If a collection is not an $O$-collection, the fact may have to be separately asserted. We propose in the cases where, for the sake of conciseness such usage is advisable, to call the class of all those collections which are not $O$-collections, $E$-collections. The symbol $E(\alpha)$ may be read at pleasure as the assertion: "The collection $\alpha$ is not an $O$-collection," or again " is an $E$-collection." Correspondingly we read the symbols : $E(x y)$ (where we also assert that " $x$ and $y$ form an $E$-pair," or "do not form an $O$-pair"); $E(x \alpha) ; E(x y B \gamma)$, etc. If $\alpha$ is not an $E$-collection, then $O(\alpha)$.

In general, we shall speak of "O-pairs," "O-triads," "O-tetrads," " $O$-n-ads," and of " $E$-pairs," "E-triads," etc., wherever our collections, whether $E$-col- 
lections or $\mathrm{O}$-collections, are restricted as to the number of elements in a way to which we wish to call attention.

10. When we simply assert $O(\alpha)$ or $E(\beta)$ of any collection, the order in which the elements of an O-collection or of an E-collection are named or considered is indifferent. The elements in question in such cases, simply do or do not belong to the collections in question without regard to the order in which the elements stand.

\section{Equivalent elements.}

11. In case two elements are such that each of them can be substituted for the other in every $O$-collection in which that other occurs, while leaving the collection in question still an $O$-collection, then these two elements are said to be equivalent each to the other or to be mutually equivalent elements. But if there exists an $O$-collection into which either of these elements enters, while the other cannot be substituted for the first, in that collection, without altering it into an $E$-collection, then the two elements are not equivalent. The equation $x=y$ means, therefore, in the present discussion not that $x$ and $y$ are identical, but simply that " either $x$ or $y$ may be substituted for the other in any $O$-collection wherein that other occurs, while the substitution leaves the collection in question still an O-collection." In case it were possible that neither $x$ nor $y$ formed a member of any O-collection, this definition would imply that they were then also equivalent. The usual properties of the relation of equivalence obviously follow from this definition : viz., $x=x$; if $x=y$, then $y=x$; if $x$, $y$, and $z$ are such that $x=y$, and $y=z$, then $x=z$. It is plain that, if $x=y$, either $x$ or $y$ can be substituted for the other in any $E$-collection in which that other occurs. For if $x=y$, it is, by definition, impossible that $E(x \alpha)$ while $O(y \alpha)$; hence, if $E(x \alpha), E(y \alpha)$ follows; and the converse is also obvious from the definition of equivalence.

12. As just pointed out, equivalent elements need not be identical. Hence, although the assertion: " $a$ is not equivalent to $b$," obviously implies the assertion: " $a$ is not the same element as $b$," - these two assertions must still be carefully distinguished, since the second of them does not imply the first. For the assertion " $a$ is not equivalent to $b$," we shall use the symbol $a \neq b$. This means that " there exists at least one $O$-collection into which one of these elements enters, while, if the other is substituted for the first in that collection, the collection in question becomes an $E$-collection."

\section{Mutually obverse elements and collections.}

13. If two elements of $\Sigma$, say $p$ and $q$, are such that $O(p q)$, then $p$ and $q$ are said to be mutually obverse elements, or obverses, each of the other. Mutually obverse elements are then such elements as together form an $O$-pair. If the assertion $O(x x)$ were true of any element, that element would be an obverse of itself. A given element may possess various obverses. 
14. If a set of $O$-pairs exists, such that, for certain existent elements of $\Sigma$, $O(a b), O(c d), O(e f), O(p q)$, etc. (this set possessing either finite or infinite multitude), and if we suppose a collection $a$ made up by the selection of one member, and of one member only, from each of these pairs, and if a collection $\beta$ is supposed to be made up out of all the remaining members of the pairs, the collections $\alpha$ and $\beta$ are said to be mutually obverse collections.

15. In consequence of the formation of the collections $\alpha$ and $\beta$ just defined, certain repetitions of elements may occur in $\alpha$, or in $\beta$, or in both. In such a case, just as in the cases mentioned above, in 3, the various repetitions of any given element are to be regarded as distinct members of the collections $\alpha$ and $\beta$ in question. Thus, if the $O$-pair $O(a b)$ is repeated, then, out of the two pairs $O(a b)$ and $O(a b)$, treated, for the purpose in hand, as distinct pairs, we can form, according to the procedure defined in 14, the collections $(a, a)$, and $(b, b)$. These collections are hereupon to be regarded, by virtue of our definition, as mutually obverse collections. Again, if the pairs $O(p q), O(x y)$, $O(m n), O(x k)$, and $O(m q)$, are given, then the collections $(p, x, m, x, m)$, and $(q, y, n, k, q)$, are mutually obverse collections, as are also any two collections that can be formed out of these same pairs by any permissible variation of the procedure defined in 14 . The order in which the members of each of the mutually obverse collections are written, is again indifferent.

16. Suppose a collection $\delta$ is first given. Let each element of this collection be such that it can be made to form an ciement of a pair of elements which (whether the elements of this pair are, or are not, repetitions of those present in other pairs), is distinct from the pair of which any other element of $\delta$ is a member. If each of the pairs thus formed is an $O$-pair, then the collection $\epsilon$, consisting of all the remaining elements of the pairs in question, is an obverse of the collection $\delta$, while $\delta$ is an obverse of $\epsilon$. Thus, if $\delta$ is the collection $(x, m, k, l, l)$, and if $O(x y), O(m j), O(k n), O(l r)$ and $O(l s)$; and if $\epsilon$ is the collection $(y, j, n, r, s)$, then the collections $\delta$ and $\epsilon$ are mutually obverse collections.

\section{Complenients and resultants.}

17. In case an element $q$ exists such that, for a given collection $\beta, O(\beta q)$ is true, the element $q$ is called a complement of $\beta$. In case $q$ and $r$ exist such that, for a given collection $\beta, O(\beta q)$ is true, while, at the same time $O(q r)$, then $r$ is called a resultant of $\beta$.

18. The properties of those collections which may be formed of the elements of $\Sigma$ are, in the main, properties determined by the existence of equivalent and of non-equivalent elements, of obverses and of resultants, together with the existence of certain laws and principles which hold valid for the system.

19. To the statement of these principles or "postulates" we now proceed. They may be classified under two heads. They are : (1) General laws to which 
all $O$-collections, in case such exist, are to conform ; and (2) Principles requiring, either conditionally or unconditionally, the existence of certain elements, and of certain collections.

\section{(1) Laws to which all O-collections conform.}

I. If $O(\alpha)$, then $O(\alpha \gamma)$, whatever collection $\gamma$ may be.

II. If, whatever element $b_{n}$ of $\beta$ be consiciered, $O\left(\delta b_{n}\right)$, and if $O(\beta)$ is also true, then $O(\delta)$.

(2) Principles requiring the existence of elements of $\Sigma$.

III. There exists at least one element of $\Sigma$.

IV. If an element $x$ of $\Sigma$ exists, then $y$ exists such that $x \neq y$.

V. Whatever pair $(p, q)$ exists, such that $p \neq q, r$ also exists such that, while both $O(r p)$ and $O(r q)$ are false, $O(p q r)$ is true.

VI. If $w$ exists such that $O(\vartheta w)$, then $v$ also exists such that $O(\vartheta v)$, and such, too, that, whatever element $t_{n}$ of $\vartheta$ be considered, $O\left(v w t_{n}\right)$.

20. These principles may be restated, with less use of symbols, thus :

I. An $O$-collection remains an $O$-collection, whatever elements or collections may be adjoined to it.

II. If a collection $\beta$, consisting wholly of elements which are complements of a collection $\delta$, is an $O$-collection, then $\delta$ itself is an $O$-collection.

III and IV (in combination). The system $\Sigma$ contains at least one pair of mutually non-equivalent elements.

V. If any pair of mutually non-equivalent elements is given, a third element of $\Sigma$ axists which forms an $O$-pair with neither of the elements of this pair, but which is such that the three elements in question together constitute an $O$-triad.

VI. If there exists any complement of a given collection $\vartheta$, then, if $w$ be such a complement, there exists a complement of $\vartheta$, viz. $v$, such that every element of $\vartheta$ is a complement of the pair $(v, w)$.

At the close of the introduction a system $\Sigma$ which conforms to all the foregoing principles, has been already pointed out.

\section{Chapter II. Elementary Consequences of 'the Principles.}

21. The elimination of obverses. If any collection $a$ is such that $x$ and $y$ exist such that $O(\alpha x)$ and $O(\alpha y)$, while $O(x y)$, then $O(\alpha)$. This follows directly from principle II, if the pair $(x, y)$ be viewed as the collection $\beta$ of that principle.

22. The correspondence of mutuclly obverse $O$-collections. If any collection $\pi$ is such that $O(\pi)$, and if a collection $\rho$ is a collection which is an obverse collection of the collection $\pi$, then $O(\rho)$. For let $p_{n}$ be any element of $\pi$. Then in $\rho$ there exists (by the definition of mutually obverse collections, as given 
in $14,15,16)$, some element $r$ such that $O\left(p_{n} r\right)$. By principle $I$, we may adjoin to the $O$-pair, $O\left(p_{n} r\right)$, all the remaining elements of $\rho$ besides $r$, and the thus enlarged collection will still remain an $O$-collection; so that $O\left(p_{n} \rho\right)$. As an analogous result holds of every other element, $p_{r}$, of $\pi$, without exception, it appears that $\pi$ consists entirely of elements each of which is a complement of the collection $\rho$. Since, however, $O(\pi)$, by principle II, $O(\rho)$.

23. The elimination of common elements. If $\eta$ and $\theta$ are mutually obverse collections; and if $x$ exists such that $O(\beta x)$ and $O(\vartheta x)$, while $y$ exists such that $O(x y)$, then $O(\beta \eta)$. For, by 22, from $O\left(\vartheta_{x}\right)$ follows $O(\eta y)$, since, by adjoining $x$ to $\vartheta$, and $y$ to $\eta$, we form the two mutually obverse collections $(x, \vartheta)$ and $(\eta, y)$. By adjunction, in accordance with principle I, from $O(\beta x)$ follows $O(\beta \eta x)$ and from $O(\eta y)$ follows $O(\beta \eta y)$. Since $O(x y)$, there follows, from $O(\beta \eta x)$ and $O(\beta \eta y)$, by principle II, as explained in 21, $O(\beta \eta)$.

24. The elimination of partial collections. If $\epsilon$ consists solely of elements which are complements of a collection $\lambda$, if $\delta$ and $\gamma$ are mutually obverse collections, and if $O(\delta \epsilon)$, then $O(\gamma \lambda)$. For $\epsilon$ by hypothesis consists of elements which are complements of $\lambda$. Let $e$ be, then, an element of $\epsilon$. Then $O(e \lambda)$, and hence, by adjunction (principle I), $O(e \gamma \lambda)$. Furthermore, $\delta$ consists wholly of elements which are complements of $\gamma$. Hence if $d$ is an element of $\delta, O(d \gamma)$ and hence, by adjunction (principle I), $O(d \gamma \lambda)$. Any element of $\epsilon$, and also any element of $\delta$, is thus a complement of $(\gamma \lambda)$. Hence the $O$-collection $O(\delta \epsilon)$ consists entirely of elements which are complements of the collection $(\gamma, \lambda)$. Hence the collection $(\gamma, \lambda)$ is itself an $O$-collection, by principle II. If $\epsilon$ reduces to a single element, $e$, then the hypotheses above stated reduce to $O(\delta e)$ and $O(\lambda e)$, while $\delta$ and $\gamma$ are mutually obverse collections, and the result then becomes identical with that of 23 . But if $\delta$ reduces to a single element $d$ and $\gamma$ to an obverse element $c$ such that $O(c d)$, then the result is that if $\epsilon$ consists solely of elements which are complements of a collection $\lambda$, and if $d$ is such that $c$ exists such that $O(c d)$, while $O(d \epsilon)$, then $O(c \lambda)$.

25. The operations of the reduction of collections through the elimination of elements and of partial collections as explained in the foregoing, will be found to be of fundamental significance throughout our procedure in what follows.

26. The existence of obverse elements. By virtue of principles III and IV, there exists $(x, y)$ such that $x \neq y$. Since $x \neq y$, it follows from the definition of equivalence (11) that there exists at least one $O$-collection into which one of these elements, say $x$, enters ; while in that collection (whether $y$ is also a member of the collection in question or not), $y$ cannot be substituted for $x$ without changing the collection into an $E$-collection. Let $\alpha$ be the collection thus characterized. Then, by hypothesis, $O(\alpha)$.

If $\alpha$ contains all the elements of $\Sigma$, occurring either once each, or in any multitude of repetitions, then a collection exists which contains all of the ele- 
ments of $\Sigma$, and which is an $O$-collection. If $\alpha$ does not contain all of the elements of $\Sigma$, nevertheless, by principle I, since $O(x)$, all of the elements of $\Sigma$ which do not appear in $\alpha$ may be adjoined to $\alpha$, and the resulting collection, say $\vartheta$, will be an $O$-collection.

Hence, in any case, there exists a collection which contains every element of $\Sigma$ (each element occurring in that collection either once only, or else repeatedly), while this collection, say $\vartheta$, is an $O$-collection.

27. Since $\vartheta$ is such that $O(\vartheta)$, every element of $\Sigma$ is a complement of $\vartheta$, by principle I. Let $w$ be any element of $\Sigma$. Since $w$ is a complement of $\vartheta$, it follows by principle VI, that $v$ exists, such that, whatever element $x$ of $\Sigma$, or of $\vartheta$, be chosen, $O(x v w)$. Since every element of $\vartheta$ is thus a complement of the pair $(v, w)$, while $O(\vartheta)$, it follows by principle II, that $O(v w)$ is true. By adjoining to the pair $(v, w)$ all the elements of $\Sigma$ which do not belong to this pair, we now have $O(\Sigma)$, an assertion according to which each element of $\Sigma$ is supposed to appear once, and without repetition, in the $O$-collection in question. Since $O(v w), v$ and $w$ are mutually obverse elements.

Since any element whatever of $\Sigma$ may be taken instead of $w$, while, each time, an element would be found to take the place here occupied by $v$, we have so far two results :

(1) The system $\Sigma$, taken in its entirety, is an $O$-collection.

(2) Every element of $\Sigma$ possesses at least one obverse.

28. A fundamental property of all pairs of mutually obverse elements hereupon comes to our notice, and is as follows: Let $b$ be any element. Let $q$ and $r$ be two obverses of $b$, so that $O(q b)$ and $O(r b)$. Hereupon let $\gamma$ be any collection such that $O(\gamma q)$; that is, let the collection $O(\gamma q)$ be any $O$-collection into which $q$, one of the obverses of $b$, enters. Then, by adjunction (principle I), we have, since $O(b r), O(b r \gamma)$; and, since $O(\gamma q), O(q r \gamma)$ (wherein we may of course change, as we here do, at pleasure, the order in which the members of the $O$-collection are written). The collection $(r, \gamma)$ is thus such that, if either of the members of the $O$-pair $O(b q)$ be separately adjoined to it, the resulting enlarged collection is each time an $O$-collection. Hence, by principle II, $O(r \gamma)$. It thus appears that, whatever the collection $\gamma$ may be, if $O(q \gamma)$, $O(r \gamma)$ follows. By a precisely analogous reasoning we could show that if $\gamma$ is such that $O(r \gamma), O(q \gamma)$ follows. Hence the two obverses of $b$ here in question, viz., $q$ and $r$, are such that either of them may be substituted for the other in any $O$-collection in which that other occurs, while still leaving that collection an $O$-collection. Hence by the definition of equivalence $q=r$. As the reasoning thus used applies to any two obverses of the same element $b$, whatever $b$ is, we have, as a result, the proposition that any two obverses of the same element are mutually equivalent elements. That is, again, if $q$ is an obverse of $b$, and $b$ is an obverse of $r$, then $q=r$. 
29. If $x=y$, while $O(q x)$ and $O(r y)$, then, by the definition of equivalence, $O(r x)$, and from $O(q x)$ and $O(r x)$ follows, by 28, the proposition $q=r$. Hence all the obverses of equivalent elements are equivalent.

30. If $u \neq v$, while $O(n u)$ and $O(m v)$, then $m \neq n$. For if $m=n$, then, by 29, $u=v$. Hence the obverses of non-equivalent elements are themselves non-equivalent.

31. It thus appears that all the obverses of the same element, or of equivalent elements, are mutually equivalent, and that non-equivalent elements cannot possess mutually equivalent obverses, still less the same obverse. Accordingly, since equivalence here means capacity for mutual substitution in $\mathbf{O}$-collections, we may henceforth let a single one of the obverses of a given element represent the whole class of these obverses, for all the purposes involved in the present discussion of $O$-collections. This uniquely selected representative of all the obverses of any element $x$, we shall henceforth regard, therefore, as the obverse of $x$, and as equivalent to the obverse of any element equivalent to $x$. We shall symbolize this single representative of all these obverses by $\bar{x}$, or, in general, by writing a bar above the symbol of the element of which at any time we define the obverse. $\bar{x}$ cannot be equivalent to the obverse of any element which is not equivalent to $x$. As the unique representative of the obverses of $\bar{x}$ we may hereupon take an element symbolized by $\bar{x}$.

32. By definition, and by $31, x=\bar{x}$; and $\bar{x}$ will henceforth be so chosen as to be identically the same element as $x$. The operation of obversion (that is, of finding, for any element $x$, the unique representative, $\bar{x}$, of the class of elements any one of which forms, with $x$, an $O$-pair), hereupon becomes an entirely univocal operation. This operation, if once repeated, is so defined as to be an operation which restores to us the original element.

33. When one collection, $\delta$, is an obverse of another collection, $\epsilon$ (see 14,15 , 16), each of these collections, by the substitution of the equivalent elements (in case such substitution is required for the purpose), may be made into a collection consisting wholly of the unique representatives of the obverses of the various members of the other collection. An obverse of the collection $\delta$, thus reduced to the form of a collection of the unique representative obverses of the elements of $\delta$, shall henceforth be symbolized, in our discussion, by $\delta$. By the symbol $\delta$ we mean, therefore, a certain chosen unique representative of all those collections any one of which is an obverse of the collection $\delta$. The collection $\vec{\delta}$ may then be so chosen as to be identical with $\delta$.

34. If $a=b$, then $O(a \bar{b})$ and $O(b \bar{a})$. For $O(a \bar{a})$ by definition $(13,31)$. Hence, since $a=b$, and since, by the definition of equivalence, we can accordingly substitute $b$ for $a$ in $O(a \bar{a})$, we have $O(b \bar{a})$. And since $O(b \bar{b})$, we have, by the substitution of $a$ for $b, O(a \bar{b})$. On the other hand, if either $O(a \bar{b})$, or $O(b \bar{a})$, is known to be true, then, by the definition of obverses, $a$ is 
an obverse of $\bar{b}$, or $b$ is an obverse of $\bar{a}$, as the case may be. But in either case, since obverses of the same element or equivalent elements are equivalent, $\bar{a}=\bar{b}$, and $a=b$.

35. A fundamental characteristic of the system $\Sigma$ is, further, the fact that: No monad is an O-collection. For if $O(x)$, then, by adjunction (principle I), $O(x x)$. By the same principle, if $O(x), O(x q)$, whatever element $q$ may be. Hence any monad $q$ is such that whether $x$, or an obverse of $x$ (namely $x$ itself), be adjoined to $q$, always $O(x q)$. Hence, by principle II, $O(q)$. Therefore if a single element $x$ exists such that $O(x)$, every element of $\Sigma$, as for instance $q$, is such that $O(q)$. Hence, by principle I, whatever collection of elements be adjoined to any element $q$ of $\Sigma$, the resulting collection is an $O$-collection. Hence (by 11), since all possible collections are thus $O$-collections, all the elements of $\Sigma$ are mutually equivalent. But this contradicts principle IV. Hence it is impossible that any monad $x$ should exist such that $O(x)$. Every monad, therefore, is an $E$-collection.

36. It will be convenient, at this point, to restate the theorems of 21-24 in the notation for obverse elements which has now been adopted:

(1) If $O(\alpha x)$ and $O(\alpha \bar{x})$, then $O(\alpha)$ (see 21).

(2) If $O(\pi)$, then $O(\bar{\pi})$ (see 22).

(3) If $O(\beta x)$ and $O(\vartheta x)$, then $O(\beta \bar{y})$ (see 23).

(4) If $O(\delta \epsilon)$, while $\lambda$ exists such that $O\left(\lambda e_{n}\right)$ for every element $e_{n}$ of $\epsilon$, then $O(\delta \lambda)$ (see 24).

(5) If $O(d \epsilon)$, while $\lambda$ exists such that $O\left(\lambda e_{n}\right)$ for every element $e_{n}$ of $\epsilon$, then $O(\overline{d \lambda})$ (see 24).

37. Any repetitions of an element which occur in an $O$-collection may be stricken out, so that the element in question occurs but a single time; and the resulting collection will still be an $O$-collection. For suppose $\alpha$ to be a collection consisting wholly of the element $a$, repeated any multitude of times. And suppose $\beta$ to be such that $O(\alpha \beta)$. From $O(\alpha \beta)$ follows by $36(2), O(\bar{\alpha} \bar{\beta})$. If any element, either of $\bar{\alpha}$, or of $\bar{\beta}$, be adjoined to the collection $(a, \beta)$ (which consists of $a$, occurring only once, with $\beta$ adjoined), it is plain that the resulting collection will be enlarged so as to constitute an $O$-collection. For $O(a \bar{a} \beta)$; and, if $b_{n}$ be any element of $\beta$, and $b_{n}$ the obverse of this element $O\left(a \beta \bar{b}_{n}\right)$. The collection $(\bar{\alpha} \bar{\beta})$, consisting entirely of complements of $(a, \beta)$, is thus an $O$-collection. Hence $O(a \beta)$, by principle II.

38. It further follows that, if $\beta$ itself is also a collection consisting solely of $a$ repeated, and if $O(\alpha \beta)$ then the collection $O(\alpha \beta)$ which now consists solely of $a$ repeated, can be reduced to $O(a a)$, and hence to $O(a)$. But $O(a)$ is impossible by 35 . Hence no collection consisting solely of repetitions of a single element can be an $O$-collection. Every such collection must be an $E$ collection. 
It still further follows that, no element is equivalent to its own obverse. For since $O(x \bar{x})$, it follows, by the definition of equivalence, that, if $x=\bar{x}, O(x x)$, Hence by $37, O(x)$. But this, by 35 , is impossible.

39. If $a$ and $b$ are such that the mutually obverse pairs $(\bar{a}, b)$ and $(a, \bar{b})$ are such that $O(\bar{a} b x)$ and $O(a \bar{b} x)$, then by 21 and 22, $O(\bar{a} b)$. Hence, by 34, $a=b$.

40. If any collection $\delta$ is such that $O(\delta)$ is false, so that $E(\delta)$ is true, it follows, from principle $I$, that if $\epsilon$ is any collection such that $\epsilon$ consists wholly of elements which belong to the collection $\delta$, while $\epsilon$ does not include all of these elements, then $O(\epsilon)$ is false. For if $O(\epsilon)$, then, since $\delta$ may be formed from $\epsilon$ by adjoining to $\epsilon$ certain elements, principle I would require that $O(\delta)$ should be true. Whatever elements, therefore, we omit from an $E$-collection, $\delta$, the remaining elements form an $E$-collection. Or, in other words, if a collection is an $E$-collection, all possible partial collections that can be formed by selecting some of its elements, and omitting others, are also $E$-collections, so that if $\epsilon$ is any such partial collection, $O(\epsilon)$ is false.

41. If $E(a \bar{b})$, then $a \neq b$. For if $a=b$, then, by 34, $O(a \bar{b})$, which contradicts the hypothesis $E(a \bar{b})$. And, on the other hand, if $a$ and $b$ are such that $a \neq b$, then $E(a \bar{b})$, for if not, then $O(a \bar{b})$, and, therefore, by $34, a=b$, which contradicts the hypothesis $a \neq b$. Thus then, if two elements, $x$ and $y$, form an $E$ pair, the obverse of either of these elements is not equivalent to the other element ; i. e., $x \neq \bar{y}$; and $\bar{x} \neq y$. Plainly, furthermore, if $E(x \bar{y})$, then $E(\bar{x} y)$, and conversely, again, if $x$ exists such that either $E(a \bar{b} x)$ or $E(\bar{a} b x)$ is true, then, by 40 , either $E(a \bar{b})$, or else $E(\bar{a} b)$ is true. But as we have just seen each of the assertions: $E(a \bar{b})$ and $E(\bar{a} b)$, implies the other, and also implies $a \neq b$. In the same way, if $E(x y z)$, then $E(x y), E(y z)$ and $E(x z)$. Hence $x \neq \bar{y}, y \neq \bar{z}$, etc. In general, if $E(\alpha)$, and if $x$ and $y$ are any two of the elements of $a$, then, by $40, E(x y)$, and hence $x \neq \bar{y}$. This is also immediately evident from principle I, and from the definition of obverses. For if $x=\bar{y}$, then $O(x y)$, and then any collection into which both $x$ and $y$ enter is, by principle $I$, an $O$-collection.

42. If $E(\alpha)$, and if $x$ be any element whatever, then either $E(\alpha x)$ or $E(a \bar{x})$ must be true. For if neither of these assertions is true, then $O(a x)$ and $O(\alpha \bar{x})$; and then, by principle II, $O(\alpha)$.

43. By 35 every monad is an $E$-collection. Let $x$ and $y$ be any two elements of the system $\Sigma$. Each of these elements possesses an obverse. Since $E(x)$, by 42 either $E(x y)$ or $E(x \bar{y})$ is true; and since $E(y)$, either $E(x y)$ or $E(\bar{x} y)$ is true.

\section{Chapter III. The $\boldsymbol{F}$-Collections.}

44. If two collections, $\beta$ and $\eta$, are such that $O(\beta \bar{\eta})$, then the collections $\beta$ and $\eta$ stand to each other in a relation which we shall also, at pleasure, express 
by the symbol $F(\beta \mid \eta)$, wherein the symbols $\beta$ and $\eta$ appear with a short vertical line between them. This symbol then, in the first place, expresses precisely the same facts that are expressed by the symbol $O(\beta \bar{\eta})$. That is the symbol $F(\beta \mid \eta)$ may at pleasure be read as the assertion: "The collection consisting of $\beta$ taken together with the collection $\eta$ (the collection which is the obverse of $\eta)$, constitutes in its totality, an $O$-collection." Since, by 22 and 33, $O(\beta \bar{\eta})$ implies $O(\bar{\beta} \eta)$, and conversely, the symbol $F(\beta \mid \eta)$ could equally well be read: - " The collection $(\bar{\beta}, \eta)$ is an $O$-collection." But the symbol $F(\beta \mid \eta)$ is especially intended to emphasize the fact that, when $O(\bar{\beta} \eta)$, and consequently when $O(\beta \bar{\eta})$, the collections $\beta$ and $\eta$ stand to each other in a relation which is mediated by the existence of their respective obverse collections. $\beta$ and $\eta$ are then collections such that each, if adjoined to the obverse of the other collection, unites with that obverse, to constitute a total collection that is an $O$-collection. Expressing this fact with a primary reference to $\beta$ and $\eta$, instead of to $\beta$ and $\bar{\eta}$, or to $\bar{\beta}$ and $\eta$, the symbol $F(\beta \mid \eta)$ may now be read as the assertion: "The collection $\beta$ forms, with the collection $\eta$, a determinate $F$-collection." The special significance of the adjective determinate will appear below. The vertical line is intended as a sort of punctuation mark, to indicate the distinction between the two collections in question.

45. If $O(\gamma)$, and if hereupon $\gamma$ be in any way exhaustively divided into two "partitions," that is, into two mutually exclusive collections of elements $\delta$ and $\epsilon$, such that $O(\delta, \epsilon)$ is the same collection as $\gamma$, it is plain from the foregoing that $F(\delta \mid \bar{\epsilon})$. So the same $O$-collection makes possible various different assertions in terms of determinate $F$-collections. If $\gamma$ is a collection of unrestricted multitude, the multitude of the possible assertions in terms of $F$-collections becomes also unrestricted.

46. The rule for transforming our assertions so that what are explicitly defined as $O$-collections shall appear in the form of explicitly designated and determinate $F$-collections, is consequently as follows: If the assertion $O(\gamma)$ is given, and if we are to express this as an assertion regarding some determinate $F$-collection, then we choose at random any partial collection $\delta$ of the elements of $\gamma$. Let $\bar{\epsilon}$ be the collection which is the obverse of the collection $\epsilon$, where $\epsilon$ consists of all the remaining members of $\gamma$, not included in $\delta$. Write $F(\delta \mid \bar{\epsilon})$, or, at pleasure, $F(\bar{\epsilon} \mid \delta)$. That is, put $\delta$ on one side and $\bar{\epsilon}$ on the other side of the vertical. The way in which the partial collections included in the parenthesis are placed, in so far as these two collections are merely considered with respect to their succeeding or preceding the vertical, is then capable of transposition at pleasure. The resulting expression is to be read, as above defined, and as an assertion in. ss of a determinate $F$-collection. Instead of $F(\bar{\epsilon} \mid \delta)$, we can equally well write $F(\epsilon \mid \bar{\delta})$, or $F(\bar{\delta} \mid \epsilon)$.

47. The rule for the inverse operations transforming an assertion regard- 
ing a determinate $F$-collection into an assertion regarding an $O$-collection is now obvious. If the assertion $F(\alpha \mid \beta)$ is given, then we first take the collection $\bar{\alpha}$, or, at pleasure $\bar{\beta}$; that is, we take the obverse collection corresponding that collection which stands on one chosen side of the vertical; and then we combine this obverse collection with that collection which stands on the other side of the vertical. Hereupon we write $O(\bar{u} \beta)$ or $O(\beta \bar{a})$, or $O(\bar{\beta} \alpha)$ or $O(\alpha \bar{\beta})$, at pleasure.

48. In case expressions such as $F(\alpha \beta x \mid \gamma \delta \bar{y} z)$, or similarly complex symbols appear, we read in this way: "The collections $\alpha$ and $\beta$, together with the element $x$, constitute a collection which, taken as a whole, forms an $F$-collection with a collection consisting of the partial collections $\gamma$ and $\delta$, and of the elements $\bar{y}$ and z." The expression just set down asserts the same as is asserted by the symbol $O(\alpha \beta x \bar{\gamma} \delta y \bar{z})$, or as is asserted by the symbol $O(\bar{\alpha} \bar{\beta} \bar{x} \gamma \delta \bar{y} z)$.

49. If a collection, $\lambda$, consists of some finite number, $n$, of elements, it is one of a set or group of $2^{n}$ collections which can be formed from the given collection by first leaving that collection unchanged, and by then transforming, in every possible way, one, two, three, $\cdots$ and finally all of the $n$ elements of $\lambda$ into their respective obverses. If one of these $2^{n}$ collections is an $O$-collection, e. g., if the original collection $\lambda$ is an $O$-collection; then the collection $\bar{\lambda}$, which is one of the set of $2^{n}$ collections, is also an $O$-collection. All of the other collections of the set are hereby required to be determinate $F$-collections. But in symbolizing these determinate $F$-collections, the arrangement of the elements is no longer wholly indifferent. One must, in each case, set upon one side of the vertical all of those elements which, in any one of the transformed collections, are obverses of elements of $\lambda$; upon the other side one must place all those elements which are identical with elements of $\lambda$. One is then to set the rearranged collection within a parenthesis, and is to write $F$ before this parenthesis. In each case one thus asserts the same fact as is asserted by $O(\lambda)$; but does so, each time, with a different stress upon the partial collections whose relations to one another are thus pointed out. By means of the determinate $F$-collections, one thus analyzes, in a particular way, the various aspects of the meaning of the assertion $O(\lambda)$. Yet each one of the determinate $F$-collections points back, infallibly, to the same pair of $O$-collections ; and also predetermines the constitution of all the other determinate $F$-collections of the same set; so that, in thus emphasizing various aspects of the meaning of the assertion $O(\lambda)$, one still never loses the power to return from one aspect, thus emphasized, to any or to all the other aspects of the same assertion. The determinate $F$-collections thus defined may be grouped in $\left(2^{n}-2\right) / 2$ pairs ; since, in general, if $F(\alpha \mid \beta)$, $F(\bar{\alpha} \mid \bar{\beta})$ is true. When we enumerate the set of determinate $F$-collections, it is sufficient to name one of each pair.

50. Indeterminate $F$-collections. It is, however, occasionally convenient to 
express simply the assertion that there exists some O-collection $O(\bar{\kappa} \lambda)$, such that the collection $(\kappa, \lambda)$ is precisely the same collection as a given collection $\eta$, that is, such that $(\kappa, \lambda)$ stands for a partition of $\eta$; while we nevertheless leave it entirely undetermined what one of the possible partitions of the collection $\eta$ it is with regard to which this assertion holds true. In this case we may write simply $F(\eta)$, a symbol which we read as the assertion: "The collection $\eta$ is an (indeterminate) $F$-collection." An indeterminate $F$-collection may prove, when its determination is specified, to be any one of the determinate $\boldsymbol{F}$-collections which correspond to the possible partitions of $\eta$. Thus, if $\eta$ is the same collection as $(\kappa, \lambda)$ and if $F(\kappa \mid \lambda)$ is true, $F(\eta)$ is true; while if we merely know that $F(\eta)$ is true, we know that some one of the assertions $F(\kappa \mid \lambda), F\left(\kappa^{\prime} \mid \lambda^{\prime}\right)$, etc., is true - where $(\kappa, \lambda)\left(\kappa^{\prime}, \lambda^{\prime}\right)$, etc., are various possible partitions of the single collection $\eta$. In the same way, if $F(\eta)$, some one of the assertions $O(\bar{\kappa} \lambda), O\left(\bar{\kappa}^{\prime} \lambda^{\prime}\right)$, etc., is true.

If, setting out from the assertion $O(\gamma)$, we consider some possible partition of the collection $\gamma$, say $(\delta, \epsilon)$, and then, instead of writing, as above (45), $F(\delta \mid \bar{\epsilon})$ we write simply $F(\delta \bar{\epsilon})$, we surrender some of the information conveyed in the original assertion $O(\gamma)$, as well as in the assertion $F(\delta \mid \bar{\epsilon})$. For it now no longer appears what determinate $F$-collection corresponds to the indeterminate $F$-collection $F(\delta \bar{\epsilon})$; and the latter assertion tells us only that some one of the possible partitions of the collection $(\delta, \bar{\epsilon})$ is such that, if it is made (e. g., the partition $\left(\delta_{1}, \delta_{2}, \bar{\epsilon}_{1}, \bar{\epsilon}_{2}\right)$, wherein $\left(\delta_{1}, \delta_{2}\right)$ is the same collection as $\delta$, and $\left(\bar{\epsilon}_{1}, \bar{\epsilon}_{2}\right)$ the same collection as $\left.\bar{\epsilon}\right)$ - then $F\left(\delta_{1} \epsilon_{1} \mid \delta_{2} \bar{\epsilon}_{2}\right)$, so that $O\left(\delta_{1} \bar{\epsilon}_{1} \bar{\delta}_{2} \epsilon_{2}\right)$. It is plain that the indeterminate $F$-collections occur in pairs. If $F(\alpha)$, then $F(\bar{\alpha})$.

51. An example will serve to distinguish more clearly the kinds of information conveyed by the assertion that a collection is a determinate and by the assertion that this collection is an indeterminate $F$-collection. Let $F(a b \mid c d)$ be true. This is equivalent to asserting $O(a b \bar{c} \bar{d})$. Any one of the possible collections which can be formed by transforming $(a, b, c, d)$ through the substitution of the obverse of one or of more of its elements, is then also a determinate $F$-collection. Thus the assertion $F(a b \mid c d)$ requires :

For the collection $(a, b, c, \bar{d})$ the assertion $F(a b d \mid c)$;

For the collection $(a, b, \bar{c}, d)$ the assertion $F(a b \bar{c} \mid d)$;

For the collection $(a, \bar{b}, c, \bar{d})$ the assertion $F(a \bar{d} \mid c \bar{b})$;

For the collection $(a, \bar{b}, \bar{c}, \bar{d})$ the assertion $F(a \bar{c} \bar{d} \mid \bar{b})$;

and so on; while each of these assertions implies, and is implied by $O(a b \bar{c} \bar{d})$.

The assertion $F(a b c d)$ does not necessarily imply any one of the foregoing determinate assertions. It indicates that, of the $2^{n}-1$ or 15 possible collec. tions other than $(a, b, c, d)$ in the set of collections producible from $(a, b, c, d)$ through the substitution of obverses, some one, and consequently (since $O(\alpha)$ implies $O(\bar{\alpha})$ ), some pair of collections, must be $O$-collections. It would 
depend upon this pair to determine how the vertical lines which define the determinate $F$-collections onght to be distributed in each of the various cases which hereupon arise. Some one of these possible distributions express the truth in each case; but the assertion $F(a b c d)$ does not tell us which one this is. Thus, if $F(a b c d)$, and if we then consider the collection $(a, \bar{b}, c, \bar{d})$, it is possible that:

$F(a \mid \bar{b} c \bar{d})$, i. e., $O(a b \bar{c} d)$, and $O(\bar{a} \bar{b} c \bar{d})$; or $F(a \bar{b} c \mid \bar{d})$, i. e., $O(\overline{a b c} c l)$, and $O(\bar{a} b \bar{c} \bar{d})$; or $F(a \bar{d} \mid \bar{b} c)$, i. e., $O(\bar{a} \bar{b} c d)$ and $O(a b \bar{c} d)$, and so on for all the other cases.

The assertion $F(a b c d)$ requires some pair of these alternative $O$-assertions or some corresponding pair of the $F$-assertions, to be true, but does not specify what pair in any of the cases in question.

The indeterminate $F$-collections, like the $O$-collections, are perfectly symmetrical. In case of a pair $(a, b)$, the alternative pairs of assertions :-

$$
\left\{\begin{array}{ll}
(1) & O(a \bar{b}) \\
& O(\bar{a} \bar{b})
\end{array} \mid(2) \begin{array}{l}
O(a \bar{b}) \\
O(\bar{a} b)
\end{array}\right\}
$$

are such that if the pair (1) of assertions are both true, the assertions (2) are both of them false. Hence $F(a b)$ can mean only that $O(\bar{a} b)$ and $O(a \bar{b})$ are both of them true; while $F(a \bar{b})$ means that both $O(a b)$ and $O(\bar{a} \bar{b})$ are true. Hence, in case of pairs of elements, the distinction between determinate and indeterminate $F$-collections vanishes: and the assertion $F(a b)$ is perfectly deter minate.

Elementary properties of the F-collections: operations and transformations.

52. A number of elementary properties of $F$-collections, and a survey of certain ways in which they may be transformed, may now be readily obtained froni the already established properties of the $O$-collections.

(1) If $F^{\prime}(x y)$ then, since $O(x \bar{y}), x=y$. See 34 .

(2) If $F(a x \mid b)$ and $F(b x \mid a)$, then $a=b$. For $O(a \bar{b} x)$ and $O(\bar{a} b x)$. See 39.

(3) If $F(\eta)$, then $F(\eta \gamma)$ where $\boldsymbol{\gamma}$ is any collection whatever (Principle I).

(4) If $F(\eta)$, then $F(\bar{\eta})$, as was already observed in connection with the definition of the $F$-collections. If $F(\beta \mid \eta)$, then $F(\bar{\beta} \mid \bar{\eta})$.

(5) If $F(\eta \mid x)$ and $F(\eta \mid \bar{x})$, then $O(\eta)$, since $O(\eta \bar{x})$ and $O(\eta x)$. The question may then arise whether $F(\eta)$ is in a given case, also true. It is here first obvious that $F(\eta)$ does not follow from $F(\eta \mid x)$ and $F(\eta \mid \bar{x})$. For instance, if $F(a b \mid x)$ and $F(a b \mid \bar{x})$, then $O(a b)$. But, if $O(a b), a=\bar{b}$; while if $F(a b), a=b$ (by (1) of the present paragraph). By 38 , however, if $O(a b)$, it is impossible that $a=b$. Hence, if $F(a b \mid x)$ and $F(a b \mid \bar{x})$, 
$F(a b)$ is false. On the other hand, if $O(\alpha)$, then by adjunction, $O(\alpha x \bar{y})$ and $O(\alpha$ ary $)$. Hence, in this case, $F(\alpha x \mid y)$ and $F(\alpha x \mid \bar{y})$. But in this case also since $O(\alpha), O(a \bar{x})$. And so $F(\alpha \mid x)$, i. e. $F(a x)$, is likewise true. If $F(\eta \mid x)$ and $F(\eta \mid \bar{x}), F(\eta)$ is accordingly possible ; but does not follow herefrom.

53. Any determinate $F$-collection remains such when transformed according to the following rule: Substitute for any element, or for any collection, which stands upon one side of the vertical, the obverse of that element or collection, transfer the obverse in question to the other side of the vertical, being careful to retain, as the result of the transfer, at least one element on each side of the vertical. Thus, if $F(x \beta \mid y \delta)$, then $F(\beta \mid \bar{x} y \delta), F(\bar{y} \beta x \mid \delta), F(x \mid \bar{\beta} y \delta)$, $F(\beta \bar{y} \bar{\delta} \mid \bar{x})$, etc., are all of them true. This is obvious, because all these expressions mean the same as $O(\bar{x} \bar{\beta} y \delta)$ or as $O(x \beta \bar{y} \bar{\delta})$.

This is called transformation by transfer. If the elements are transferred by this rule except that all the elements are permitted at the end to stand upon one side of the vertical, the vertical can then be omitted; but the resulting collection must be regarded as thus transformed into an $O$-collection.

54. If $F(x \beta \mid \bar{x} \gamma)$ then $F(\beta \mid \bar{x} \gamma)$ and $F(x \beta \mid \gamma)$. If $F(x \beta \mid \bar{\beta} \gamma)$, then $F(x \beta \mid \gamma)$ and $F\left(x \mid \bar{\beta}_{\gamma}\right)$. That is, if the obverse of an element or of a collection, which stands on one side of the vertical, itself stands on the other side of the vertical, then either of the two mutually obverse collections ar elements may be stricken out (by 37). For if $F(x \beta \mid \bar{x} \gamma)$, then $O(x \beta x \bar{\gamma})$, and so $O(\beta x \bar{\gamma})$. Hence $F^{\prime}(\beta x \mid \gamma)$ and $F(\beta \mid \bar{x} \gamma)$. This is called a transformation by means of the omission of superfluous obverses; and the procedure obviously applies to collections as well as to elements.

55. If $F(\alpha \mid \beta)$, and if all the elements of $\alpha$ are mutually equivalent, and all the elements of $\beta$ are mutually equivalent, then all the elements of the collection are mutually equivalent.

For $O(\alpha \bar{\beta})$. Let $a$ be one of the elements of $\alpha$ and $b$ of $\beta$. Then, by 36, $O(\alpha \bar{\beta})$ reduces to $O(a \bar{b})$, whence follows, by $34, a=b$.

It is now obvious that any repetitions of an element which occur upon one side of the vertical in a determinate $F$-collection may be stricken out; and also that, by virtue of principle $I$, any element may be added to that collection which stands upon either side of the vertical, so that, if $F(\alpha \mid \beta), F(\alpha y \mid \beta)$ and $F(\alpha \mid \beta y)$, where $y$ is any element.

\section{Elimination-theorems for $F$-collections.}

56. If two collections $\beta$ and $\delta$ are such that there exists a collection $\pi$ such that $F(\delta \mid \pi)$, while, for every member $y_{r}$ of the collection $\pi, F\left(y_{r} \mid \beta\right)$, then $F(\beta \mid \delta)$.

For, if $F(\delta \mid \pi)$, then $O(\delta \bar{\pi})$. And if $F\left(y_{r} \mid \beta\right)$, then, for every member 
$\bar{y}_{r}$ of the collection $\bar{\pi}$ it is true that $O\left(\bar{y}_{r} \beta\right)$. Substituting for the symbol $\bar{\pi}$ the symbol $\epsilon$, and for $\bar{y}_{r}$ (the representative symbol for any member of $\bar{\pi}$ ), the symbol $x_{r}$ (as the representative symbol for any member of the collection now called $\epsilon$ ), we have $\delta$ and $\beta$ such that there exists a collection $\epsilon$ such that $O(\delta \epsilon)$, while, for every member $x_{r}$ of $\epsilon, O\left(x_{r} \beta\right)$. Hence, by 36 , and by virtue of the properties of $O$-collections pointed out in $24, O(\delta \bar{\beta})$. Hence $F(\delta \mid \beta)$ or $F(\beta \mid \delta)$.

57. In case the collection $\delta$ reduces to the single element $d$, the theorem assumes the following form :

If any collection $\beta$ forms a determinate $F$-collection with every member of $\pi$, separately considered, while the collection $\pi$ taken as an entirety, is such as to form a determinate $F$-collection with an element $l$, then $\beta$ forms a determinate $F$-collection with $d$.

This theorem permits the elimination of $\pi$, in case $F(d \mid \pi)$, and in case the set of determinate $F$-collections $F\left(y_{r} \mid \beta\right)$ is given, where $y_{r}$ is a variable for which every element of $\pi$ may separately be substituted.

58 . If $\pi$ reduces to a single member $y$, we have the result of 56 reduced to the form :

If two collections $\beta$ and $\delta$ are such that there exists an element $y$ such that $F(y \mid \beta)$ and $F(y \mid \delta)$, then $F(\beta \mid \delta)$.

This last result furnishes a means for the direct elimination of an element $y$ common to two determinate $F$-collections, in case $y$ stands alone, on one side of the vertical, in each collection. Here too we deal with a type of transitivity whose consequences are of great importance.

59. If $F(x \beta \mid y \gamma)$, and $F(y \beta \mid z \gamma)$, then $F(x \beta \mid z \gamma)$. For, by transfer (53), from $F(x \beta \mid y \gamma)$ follows $F(x \beta \bar{\gamma} \mid y)$. And from $F(y \bar{\beta} \mid z \gamma)$ follows $F(\bar{\beta} z \gamma \mid y)$. Hence, by 58 , we can eliminate $y$, and thus we obtain $F\left(x \beta \bar{\gamma} \mid \bar{\beta}_{z \gamma}\right)$. By 54 , we may hereupon transform this $F$-collection by striking out $\bar{\beta}$ from the right side of the vertical (since $\beta$ occurs on the left side), and $\bar{\gamma}$ from the left side (since $\gamma$ occurs on the right side). We thus obtain $F(x \beta \mid z \gamma)$, which was to be proved.

The transformations and the type of elimination here used are typical of the methods which are to be employed in considering and in combining $F$-collections. These methods correspond to the adjunctions and eliminations already used in case of $O$-collections.

60. The result of 59 is the principal theorem relating to the transitivity of the relations involved in $F$-collections. Its importance justifies a proof directly in terms of the properties of $O$-collections.

If, namely, $F(x \beta \mid y \gamma)$, then $O(x \beta \bar{y} \bar{\gamma})$. From this follows, by adjunction, $O(x \beta \bar{\gamma} \bar{y} \bar{z})$. If $F(y \beta \mid z \gamma)$, then $O(y \beta \bar{z} \bar{\gamma})$. From this follows, by adjunction, $O(x \beta \bar{\gamma} y \bar{z})$. From the two $O$-pentads, thus formed through adjunction, follows, by principle II, $O(x \beta \bar{\gamma} \bar{z})$. Hence $F(x \beta \mid \gamma z)$. 
61. The hypothesis $F(x \beta \mid y \gamma)$ sets $x$ in a determinate dyadic relation $R$ to $y$; and this relation is unsymmetrical, since $y$ does not stand in this relation to $x$. The hypothesis $F(y \beta \mid z \gamma)$ sets $y$ in this same relation $R$ to $z$. The conclusion sets $x$ in the relation $R$ to $z$. The relation in question is therefore transitive. But the unsymmetrical transitive dyadic relation in question has been entirely derived from the wholly symmetrical relations defined by the $O$-collections.

62. If $\beta, \gamma$, and $\delta$ are given collections, and if $y$ and $e$ are given elements, such that $F(y \beta \mid \delta)$ and $F(e \delta \mid \beta)$, while $F(e \mid y \gamma)$, then $F(\gamma \delta \mid \beta)$.

For by transfer (53), from $F(y \beta \mid \delta)$ and $F(e \mid y \gamma)$ follow the two assertions $F(y \mid \delta \bar{\beta})$ and $F(y \mid e \bar{\gamma})$. By 58 , it follows that $F(\delta \bar{\beta} \mid e \bar{\gamma})$; whence follows again by transfer $F(\bar{\beta} \gamma \delta \mid e)$. From $F(e \delta \mid \beta)$ follows, by transfer, $F(e \mid \beta \bar{\delta})$. From this and $F(\delta \bar{\beta} \gamma \mid e)$ follows, by $58, F(\beta \bar{\delta} \mid \gamma \delta \bar{\beta})$. By omission of superfluous obverses (54), we obtain $F(\beta \bar{\delta} \mid \gamma)$ and $F(\beta \mid \gamma \delta)$.

63. If $F(x \mid \beta)$ and $F(y \mid \beta)$, while $F(x y \mid \delta)$ then $F(\delta \mid \beta)$.

This follows directly from 56 , in case the collection $\pi$ of that theorem reduces to the pair $(x, y)$.

64. If $\alpha, \delta, \epsilon$ are collections, and $b$ and $c$ are elements, such that (1) $F(b \alpha \mid \delta)$ and (2) $F(c \alpha \mid \delta)$, while (3) $F(b c \mid \epsilon)$, then $F(\delta \mid \alpha \epsilon)$.

For by (1) $F(b \alpha \mid \delta)$ while by $(2), F(\alpha \mid \delta \bar{c})$. Let $b=y$, and $c=\bar{e}$. Then $F(y \alpha \mid \delta)$, while $F(\alpha \mid \delta e)$; and, by (3), $F(y \mid \epsilon e)$, i. e., $F(e \mid y \bar{\epsilon})$.

Hence, by $62, F(\bar{\epsilon} \delta \mid \alpha)$. Whence follows $F(\alpha \epsilon \mid \delta)$. That is, if two collections $\alpha$ and $\delta$ are such that if either $b$ or $c$ be separately adjoined to $\alpha$, the resulting collection forms an $F$-collection with $\delta$, and if the pair $(b, c)$ forms an $F$-collection with $\epsilon$, then if $\epsilon$ itself be adjoined to $\alpha$, the resulting collection forms an $F$-collection with $\delta$.

\section{Chapter IV. $F$-triads, mediators and antecedents.}

65. What elements exist in the system $\Sigma$ we have as yet but very imperfectly investigated. Yet before we proceed to this investigation, it will prove convenient to outline the general character of the order which is possible in the system $\Sigma$, so far as we have yet developed this order. The fact that given elements do or do not belong to a certain $O$-collection, or do or do not constitute an $O$ collection, is one which appears directly to establish no sort of order amongst the elements of $\Sigma$. The relation in which various elements stand to one another when they belong to the same $O$-collection, is so far absolutely symmetrical, and nothing can be said of one member of such a collection which is not asserted of everyone of the others, so far as this collection is concerned.

But the fact that every element of $\Sigma$ possesses an obverse, enables one to establish relations between certain elements, or sets of elements, relations which are due to the further fact that given elements may enter into $O$-collections with the 
obverses of certain other elements. The consequence of this is that $F$-collections are definable. Let us provisionally assume that a large variety of $F$-collections exist in $\Sigma$.

66. $F$-collections, if indeterminate, are, like $O$-collections, of a wholly symmetrical structure, and their members are so far undistinguished from one another. But the members of a determinate $F$-collection are no longer so symmetrically disposed. It is indeed true that the order in which the elements in the set of an $F$-collection on each side of the vertical are considered, is indifferent. Nor does it make any difference which set is written before or after the vertical. But if $F(\beta \mid \gamma)$, then, in general, any element $x$ of $\beta$ is related to any element $y$ of $\gamma$ in a way which is not reciprocated. For $x$ is related to $y$ as that element which, in combination with some collection $\beta^{\prime}$ of companion elements, forms an $F$-collection with $y$, when $y$ is combined with a collection $\gamma^{\prime}$ of elements $\left(\beta^{\prime}\right.$ being the collection of the other elements of $\beta$ besides $x, \gamma^{\prime}$ being the collection of the other elements of $\gamma$ besides $y$ ). This relation, if read in the other direction, changes, in general, its character, and so is an unsymmetrical relation.

But, as 59 has shown us, this unsymmetrical dyadic relationship is transitive. In terms of this relation certain sets of the elements may be ordered and so arranged in series like points on a line.

67. The fundamental form of such series becomes manifest if we pay attentiou to those cases of the much more general theorems regarding $F$-collections, which appear as special results if we consider only triads of elements.

In this case if, for example, $F(a c \mid b)$ or $F(c a \mid b)$, that is, if $O(a c \bar{b})$, we shall call $b$ " the mediator between $a$ and $c$," or, where that is more convenient, the "mediator of the pair $(a, c)$." The "mediator" of a pair is accordingly an element whose obverse forms an $O$-triad when adjoined to that pair. If $O(p q r)$, then $F(p q \mid \bar{r})$, so that the obverse of any member of an $O$-triad is the mediator of the pair formed by the other elements of that triad.

The relation of the mediator to the elements which it mediates may be treated at pleasure either as a triadic or as a dyadic relation. In order to treat it as a dyadic relation we may take account of the fact that, if $F(a c \mid b), b$ is in a certain relation to $a$ with respect to $c$, and is in a certain relation to $c$ with respect to $a$. This aspect of the matter may become especially important in case we deal with a number of triads in which the mediators are any elements whatever, while all the pairs mediated have a common element $y$. Such pairs appear if $F(y m \mid q), F(y n \mid r), F(y o \mid s)$, etc. In all such cases $q$ has the same relation to $m$ that $r$ has to $n$, and that $s$ has to $o$, etc., since $q$ is in a given relation to $m$ with respect to $y$; and the same holds true in the other instances in question.

68. If $F(y q \mid p)$, we may, whenever that is convenient, first select one element of the pair $(q, y)$, say the element $y$, and thereupon say that $p$ is in a relation 
to $q$ which we shall call the relation: "antecedent of $q$ with respect to $y$." The element $y$ we may hereupon call the "origin" from which the relation is defined or reckoned. Equally, if we choose the element $q$ as our origin, we can say that $p$ is in the relation to $y$ of being "an antecedent of $y$ with respect to $q$ ". We shall symbolize the assertion " $p$ is antecedent of $q$ with respect to $y$," by the expression $p-<_{y} q$ or $q>>p$; and we can also at pleasure read either of these expressions thus: " $q$ is a consequent of $p$ with respect to $y$." This expression means precisely the same as the expression $F(y q \mid p)$, or as the expression $O(y q \bar{p})$. Expressing the facts in the new way, as involving the relation of an antecedent to a consequent, has merely the advantage of bringing out certain aspects of the situation which will be conveniently expressible in terms of a dyadic relation - a relation which, as already pointed out, will prove to be unsymmetrical and transitive and, therefore, useful for the definition of serial order amongst certain specially selected elements of $\Sigma$. The equivalence of meaning of the three expressions: $O(q y \bar{p}), F(q y \mid p)$, and $p<_{y} q$, enables us at once to see how superficial is the difference between symmetrical and unsymmetrical relations. All that any one of these three expressions asserts is that the two perfectly symmetrical $O$-collections $O(q y x)$ and $O(x p)$ (where $x=\bar{p}$ ), both exist as collections of the elements of $\Sigma$.

69. It is of course expressly true that the relation of antecedent to consequent, or of consequent to antecedent, has meaning only with reference to a given origin. It is this origin which gives "sense" to the pair $(p q)$ in the expression $p<_{y} q$. On the other hand, if a question arises as to whether the " sense," or asymmetry, of the dyadic relation here in question, is a fundamental fact, or is unanalyzable - this question is answered in advance by our derivation of the whole asymmetry from the perfectly symmetrical properties which characterize the various members of any $O$-collection.

The system $\Sigma$, as we shall hereafter see, includes elements whose relations are precisely the ones which are of the most fundamental importance in all the exact sciences. The customary procedure of these sciences may be said, in the main, to involve the definition of these relations in terms of the relation of antecedent and consequent. Wherever a linear series is in question, wherever an origin of coördinates is employed, wherever "cause and effect," "ground and consequence," orientation in space or direction of tendency in time are in question, the dyadic asymmetrical relations involved are essentially the same as the relation here symbolized by $p<_{y} q$.

This expression, then, is due to certain of our best established practical instincts and to some of our best fixed intellectual habits. Yet it is not the only expression for the relations involved. It is in several respects inferior to the more direct expression in terms of $O$-relations. The range of its efficacy as an expression will become clearer hereafter. When, in fact, we attempt to 
describe the relations of the system $\Sigma$ merely in terms of the antecedent-consequent relation, we not only limit ourselves to an arbitrary choice of origin, but miss the power to survey at a glance relations of more than a dyadic, or triadic character.

Properties of the relation of mediator, antecedent, and consequent.

70. If $F(a b \mid c)$, and $a=b$, then $c=a=b$. For this is a special case of the principle proved in 55.* Furthermore, if $p<_{y} q$ and $q<_{y} p$, then $p=q$, since this is but another expression of 52 (2). Consequently, if $p \neq q$, and $p<_{y} q$, then $q<_{y} p$ is false. The relation of antecedent to consequent, if it obtains at all between a pair of non-equivalent elements, is therefore in that case inconvertible; hence the relation becomes totally asymmetrical so soon as it is confined to pairs of non-equivalent elements.

71. If $F(a \mid b y)$ and $F(b \mid c y)$, then $\mid$ If $a<_{y} b$ and $b<_{y} c$, then $F(a \mid c y)$ and $F(b \mid a c)$. $a<_{\nu} c$; and $b<_{a} c$.

The proof is as in 59. Thus from $F(b \mid a \bar{y})$, and $F(b \mid c y)$ follows $F(a \bar{y} \mid c y)$ and $F(a \mid c y)$. From $F(y \mid a \bar{b})$ and $F(y \mid b \bar{c})$ follows $F(a \bar{b} \mid b \bar{c})$; and consequently $F(b \mid a c)$. The form of the theorem stated on the right is simply a direct translation into the symbolism of the relation of antecedent and consequent. The latter relation is thus shown to be transitive.

72. If $F(x \mid a b)$ and $F(x \mid b c)$, while $F(b \mid a c)$, then $x=b$. For since $F(x \mid b c)$ while $F(b \mid c a)$, we have $F(c \mid x \bar{b})$ and $F(c \mid b \bar{a})$. Whence follows $F(x \mid b \bar{a})$; that is $F(b \mid a x)$. From $F(x \mid a b)$ and $F(b \mid a x)$ follows, by 52 (2) $x=b$.

In other words, if $x \neq b$, and $b$ is a mediator of the pair $(a, c)$, it is impossible that $x$ should be at once a mediator of the two pairs $(a, b)$ and $(b, c)$.

73. If $F(x \mid a b)$ and $F(y \mid a b)$ and $\quad$ If $x<_{a} b$ and $y<_{a} b$ and also $F(d \mid x y)$, then $F(d \mid a b)$. $d<_{x} y$, then $d<_{a} b$.

This follows directly from 63 and expresses the fact that a mediator of two elements which, with respect to a given origin, are antecedents of the same element, is itself an antecedent of that element with respect to the same origin.

74. If $F(y b \mid d), \quad F(y c \mid e)$ and If $d<_{y} b$ and $e<_{y} c$, while $F(e d \mid b)$, then $F(c d \mid b)$. $b<_{d} e$, then $b<_{d} c$.

This is a special case of the proposition proved in 62 . If two pairs, $(b, y)$ and $(c, y)$, have a common element $y$, and if each pair forms a determinate $F$-triad when a term $d$ (in one case), or $e$ (in the other case) is set on the opposite side of the vertical, and if the member $b$ of the one pair is a mediator of $e$ and $d$, while $d$ is the third member of the $F$-triad in which $b$ occurs, then $b$ is a mediator of $d$ and of $c$, where $c$ is the remaining member of the other pair $(c, y)$.

* This theorem is used by KEYPE as a fundamental principle in defining the relation here called that of Mediator. 
Otherwise: If, with respect to a common origin $y$, the elements $d$ and $e$ are, respectively, antecedents of the elements $b$ and $c$, so that $d$ is antecedent of $b$ and $e$ of $c$, then, if $b$ is a mediator of $d$ and $e, b$ is also a mediator of $c$ and $d$.

75. If $F(a b \mid d), F(a c \mid d)$ and $F(b c \mid e)$, then $F(a e \mid d)$.

This follows from 64 .

That is, whatever element is mediator of two pairs which have an element in common, is mediator of the pair composed of the common element and of any mediator of the pair, formed by the elements which, belonging to the original pairs are not common to them. 'Otherwise, whatever element is, with respect to a given origin, an antecedent of each of a pair of elements, is antecedent of any mediator of this pair.

76. Any element $a$ is a mediator between any element $y$ and the obverse of $y$; and any element $y$ is a mediator between itself and any other element $a$.

For $O(\bar{u} y \bar{y})$ and $O(a y \bar{y})$.

77. For any origin $y$, it is true, that $y$ is an antecedent of itself and of every other element, including $\bar{y}$; while any element $a$ is an antecedent of itself and of $\bar{y}$.

For, $O(\bar{y} y a), O(\bar{y} y y)$ and $O(\bar{y} y \bar{y})$, while $O(\bar{a} a y)$ and $O(\bar{a} y \bar{y})$.

78. If $a<_{y} b$, then $b<_{y} \bar{a}$.

For $O(\bar{a} b y)$, and hence $F(\bar{b} \mid \bar{a} y)$.

79. Whatever element is, with respect to a given origin $y$, an antecedent of every member of an $O$-collection except one, is also an antecedent of the obverse of this excepted element.

Let $\pi$ be any collection, and $e$ such an element that $O(\pi e)$. Then, by this hypothesis $F(\pi \mid \bar{e})$. Let $q$ be an element such that $F\left(q \mid x_{r} y\right)$ is true of every element $x_{r}$ of $\pi$ so that $q<_{y} x_{r}$. Then, by transfer, $F\left(q \bar{y} \mid x_{r}\right)$. Hence the collection $(q, \bar{y})$ forms a determinate $F$-collection, when set on oue side of the vertical, with any element of the determinate $F$-collection $F(\pi \mid \bar{e})$ on the other side of the vertical, except the element $\bar{e}$. Hence, by 57 , it is also true that $F(q \bar{y} \mid \bar{e})$. Hence $F(q \mid y \bar{e})$. Hence $q \prec_{y} \bar{e}$.

80. If, with respect to $y, q$ is an antecedent of every member of a given $O$-collection, then $q$ is equivalent to $y$. For $q$ is in any case an antecedent, with respect to the origin $y$, of every member of the collection except any member $e$. Hence $q<_{y} \bar{e}$. But, by the present hypothesis, $q<_{y} e$ is also true. Hence $O(\bar{q} y e)$ and $O(\bar{q} y \bar{e})$. Hence $O(\bar{q} y)$. Hence $q=y$.

81. Whatever element is, with respect to a given origin, a consequent of every member of an $O$-collection except one, is also a consequent of the obverse of this excepted element.

Suppose $\pi$ such that $F(\pi \mid \bar{e})$ as before. And suppose that, for every element $x_{r}$ of $\pi, x_{r}<_{1 /} q$, so that $F\left(x_{r} \mid y q\right)$. By the same reasoning as that of the last theorem $F(\bar{e} \mid q y)$; and hence $\bar{e}<_{y} q$. 
82. If $q$ is a consequent of every member of the collection, then it is also true that $e<_{\nu} q$; whence $O(q y e)$ and $O(q y \bar{e})$. Hence $O(y q)$, and consequently $q=\bar{y}$.

Chapter V. The existence of elements. chains. Resultants. CONJUGate Pairs of Resultants. THE algebra of logic.

83. In discussing, in the foregoing, the most characteristic relations of the elements of $\Sigma$, we have tacitly and provisionally assumed that elements may exist in sufficient variety to exemplify these relations. Our existential principles have so far been used mainly to establish the existence of pairs of obverse and of non-equivalent elements. We must now proceed to survey, more in detail, the actual structure of the system $\Sigma$.

84. By principles III and IV, the system $\Sigma$ contains a pair of non-equivalent elements, say $(x y)$. By 26 , the pair $(\bar{x}, \bar{y})$ also exists. It is however in so far possible that $x=\bar{y}$, and hence that $y=\bar{x}$. In that case the members of the pair $(x, y)$ become respectively equivalent to the members of the pair $(\bar{y}, \bar{x})$, or, again, to the members of the pair $(x, \bar{x})$. Were the system $\Sigma$ to consist merely of the single pair of mutually non-equivalent and mutually obverse elements, $(x, \bar{x})$, principles I, II, III, IV, and VI would all of them be true of the system as thus restricted. For $O(x \bar{x})$; and if, to this collection, we add any collection $\gamma$, consisting either of $x$, or of $\bar{x}$, repeated any multitude of times, or again consisting both of $x$ and of $\bar{x}$, in any combination, each occuring any multitude of times - in any case $O(x \bar{x} \gamma)$. Hence principle $I$ is satisfied. On the other hand since $O(x \bar{x})$, while, by $35,37, E(x x)$, and $E(\bar{x} \bar{x})$ - the nonequivalence of $x$ and $\bar{x}$ can be readily established, without taking account of any collections except those into which $x$ and $\bar{x}$ either jointly or severally enter. In order that a collection $\beta$ shall consist altogether of complements of $\delta$, while $O(\beta)$ is true, it is necessary, in case $\Sigma$ contains only $x$ and $\bar{x}$, that $\beta$ should include both $x$ and $\bar{x}$; while $\delta$ (which, by hypothesis is such that $O\left(\delta b_{n}\right)$ for every element $b_{n}$, of $\beta$ ), must then also include both $x$ and $\bar{x}$. Hence if $O(\beta), O(\delta)$ follows. Hence principle II would hold true if the system $\Sigma$ consisted only of the pair $(x, \bar{x})$. Principles III and IV would obviously hold true of the same system. And considered with reference to $x$, its obverse, $\bar{x}$, is an element satisfying the requirements of principle $\mathrm{VI}$; while the same holds true of $x$ when it is considered with reference to $\bar{x}$. All the principles except $V$ would therefore be satisfied if the system $\Sigma$ consisted of the single pair $(x, \bar{x})$.

\section{Chains of elements, defined by recurrence.}

85. Principle V, however, is not satisfied by the existence of a single pair of elements, such as $(x, \bar{x})$. For, if the pair $(x, \bar{x})$ exists, then (since $x \neq \bar{x}$ by 
$38)$, it follows that principle $\mathrm{V}$ demands the existence of $r_{1}$ such that $E\left(r_{1} x\right)$ and $E\left(r_{1} \bar{x}\right)$. These two assertions require that $r_{1} \neq \bar{x}$ and $r_{1} \neq x$ (by 41). Since $r_{1} \neq \bar{x}$, there exists an element which we will next symbolize by $\bar{r}_{2}$, and which, by virtue of principle $\mathrm{V}$, is such that $E\left(r_{1} \bar{r}_{2}\right), E\left(\bar{r}_{2} \bar{x}\right)$, and $O\left(r_{1} \bar{r}_{2} \bar{x}\right)$. The obverse of $\bar{r}_{2}$ also exists. Since $E\left(r_{1} \bar{r}_{2}\right)$ it follows by 41, that $r_{2} \neq r_{1}$; and for the precisely analogous rwason, $r_{2} \neq \bar{x}$. At the same time $F\left(r_{2} \mid r_{1} \bar{x}\right)$. Since $r_{2} \neq \bar{x}$, there also exists, by principle $\mathrm{V}$, an element $\bar{r}_{3}$, such that $E\left(\bar{r}_{3} r_{2}\right)$ and $E\left(\bar{r}_{3} \bar{x}\right)$ while $O\left(r_{2} \bar{r}_{3} \bar{x}\right)$. The obverse $r_{3}$ of $\bar{r}_{3}$, therefore, also exists ; and is such that $r_{3} \neq r_{2}, r_{3} \neq \bar{x}$, and $F\left(r_{3} \mid r_{2} \bar{x}\right)$. The procedure whereby the elements $r_{1}, r_{2}$, and $r_{3}$ have been defined is obviously a recurrent one. Repeatedly applied it defines a chain of elements : $\left(r_{1}, r_{2}, \ldots, r_{n}, \ldots\right)$, whereof any finite number $n$ may be at pleasure defined in a determinate order. These elements, together with their obverses $\left(\bar{r}_{1}, \bar{r}_{2}, \ldots, \bar{r}_{n}, \ldots\right)$, all exist in $\Sigma$, and possess the following properties :

$$
\begin{gathered}
x \neq r_{1} ; r_{1} \neq r_{2} ; r_{2} \neq r_{3} ; \cdots ; r_{n-1} \neq r_{n} ; r_{n} \neq \bar{x} . \\
F\left(r_{1} \mid x \bar{x}\right), F\left(r_{2} \mid r_{1} \bar{x}\right), F\left(r_{3} \mid r_{2} \bar{x}\right), \ldots, F\left(r_{n} \mid r_{n-1} \bar{x}\right) . \\
F\left(\bar{r}_{1} \mid x \bar{x}\right), F\left(\bar{r}_{2} \mid \bar{r}_{1} x\right), F\left(\bar{r}_{3} \mid \bar{r}_{2} x\right), \cdots, F\left(\bar{r}_{n} \bar{r}_{n-1} x\right) . \\
\bar{x} \neq \bar{r}_{1} ; \bar{r}_{1} \neq \bar{r}_{2} ; \bar{r}_{2} \neq \bar{r}_{3} ; \cdots ; \bar{r}_{n-1} \neq \bar{r}_{n} ; \bar{r}_{n} \neq x .
\end{gathered}
$$

The expressions (3) and (4) follow directly from the truth of (1) and of (2), by the definitions of the $F$-collections, and of the relations of equivalent and of obverse elements, and by $30,36(2)$, and $52(4)$.

From these conditions it further follows that, if $m$ and $n$ are (for the moment) viewed, not as symbols for elements of $\Sigma$, but as purely numerical marks or indices, serving to distinguish the ordinal positions of different members of the chain $\left(r_{1}, r_{2}, \cdots, r_{m}, \cdots, r_{n}, \cdots\right)$, and if $m$, in the series of natural numbers, precedes $n$, then :

$$
\begin{aligned}
& F\left(r_{n} \mid r_{m} \bar{x}\right) ; \text { and } r_{n} \neq r_{m} . \\
& F\left(\bar{r}_{n} \mid \bar{r}_{m} x\right) ; \text { and } \bar{r}_{n} \neq \bar{r}_{m} .
\end{aligned}
$$

For, by the laws, of the construction of the chain, $F\left(r_{m+1} ; r_{m} \bar{x}\right)$, and $F\left(r_{m+2} \mid r_{m+1} \bar{x}\right)$. Hence $F\left(r_{m+2} \mid r_{m} \bar{x}\right)$, by 71 . In the same way, since $F\left(r_{m+3} \mid r_{m+2} \bar{i}\right)$, there follows $F\left(r_{m+3} i r_{m} \bar{x}\right)$. And the same process of elimination can be repeated any number of times, so that, in fine, $F\left(r_{n} \mid r_{n} \bar{x}\right)$. Meanwhile, by the principle proved in $72, r_{m+2} \neq r_{m}$. In fact, if we suppose $r_{m+2}=r_{m}$, we have $F\left(r_{m+1} \mid r_{m} \bar{x}\right)$ by construction, as well as $F\left(r_{m+2} \mid r_{m+1} \bar{x}\right)$. By the substitution of equivalents we therefore obtain :

$$
F\left(r_{m+1} \mid r_{m} \bar{x}\right) \quad \text { and } \quad F\left(r_{m} \mid r_{m+1} \bar{x}\right) \text {. }
$$


Whence follows, by $52(2), r_{m+1}=r_{m}$, which contradicts the conditions stated in the expressions (1). Hence $r_{m+2} \neq r_{m}$. In the same way we can prove that $r_{m+3} \neq r_{m}$; and in general, $r_{n} \neq r_{m}$, where $n$ is any ordinal number that follows $m$. Hence the expressions (5) are proved true; and the expressions (6) follow therefrom by considering the obverses of the elements occurring in (5). All the members of the chain $\left(r_{1}, r_{2}, \ldots, r_{n}, \cdots\right)$ are such that $F\left(r_{n} \mid r_{1} \bar{x}\right)$.

To sum up: The requirements of principle $\mathrm{V}$ include the assertion that, since the pair $(x, \bar{x})$ exists there must also exist the two distinct chains of elements :

$$
\left(r_{1}, r_{2}, \cdots, r_{n}, \cdots\right) \text { and }\left(\bar{r}_{1}, \bar{r}_{2}, \ldots, \bar{r}_{n}, \cdots\right) \text {. }
$$

No two of the elements of either one of these chains are mutually equivalent. If any element of the first chain, as $r_{m}$, were equivalent to a member of the second chain, say $\bar{r}_{n}$, we should have, since, by the foregoing:

$$
F\left(r_{m} \mid r_{1} \bar{x}\right) \quad \text { and } \quad F\left(\bar{r}_{n} \mid \bar{r}_{1} x\right),
$$

the consequence: $F\left(r_{m} \mid r_{1} \bar{x}\right)$ and $F\left(r_{m} \mid \bar{r}_{1} x\right)$. By elimination would follow $F\left(r_{1} \bar{x} \mid \bar{r}_{1} x\right)$; whence would follow $O\left(r_{1} x\right)$ and so $r_{1}=x$, a result which is rendered impossible by the conditions that define $r_{1}$. Hence no element of either chain can be equivalent to any member either of the same chain, or of the other chain; and all the elements of both chains are non-equivalent both to $x$ and to $\bar{x}$. Since, if any element $r_{n}$ exists in the first chain, $r_{n+1}$ also exists (while $\bar{r}_{n+1}$ also belongs to the other chain), the two chains contain each an infinite number of non-equivalent elements. The system $\Sigma$ consequently contains an infinite number of mutually non-equivalent elements.

86. Herewith, however, the requirements of principle $\mathrm{V}$ are by no means exhausted. For since no two elements of either chain are mutually equivalent, any two successive elements of each chain, as, for instance, $r_{m}$ and $r_{m+1}$, are such that, by principle $\mathrm{V}, \bar{s}_{1}$ exists such that $E\left(r_{m} \bar{s}_{1}\right)$ and $E\left(r_{m+1} \bar{s}_{1}\right)$, while $O\left(r_{m} r_{m+1} s_{1}\right)$. In this case $F\left(s_{1} \mid r_{m} r_{m+1}\right)$, while $s_{1} \neq r_{m}$ and $s_{1} \neq r_{m+1}$. A new recurrent process is thus defined, a process which can be employed to define a chain $\left(s_{1}, s_{2}, \cdots, s_{j}, \cdots\right)$ where the subscripts again have the significance of the ordinal numbers.

By 72, no two elements of this chain can be mutually equivalent. Every member $s_{j}$ of this new chain must be non-equivalent to any member of the chain $\left(r_{1}, r_{2}, \cdots, r_{m}, \cdots\right)$, as well as to any member of any chain $\left(s_{1}^{\prime}, s_{2}^{\prime} \cdots, s_{l}^{\prime}, \cdots\right)$, or $\left(t_{1}, t_{2}, \cdots, t_{h}, \cdots\right)$, such as can be formed by taking account of those pairs: $\left(r_{1}, r_{2}\right)$, $\left(r_{3}, r_{4}\right)$, etc., which are different from the pair $\left(r_{m+1}, r_{m}\right)$, and by applying principle $\mathrm{V}$ recurrently to them. In general, if $F\left(e \mid r_{m} r_{m+1}\right)$, and $F\left(g \mid r_{n} r_{n+1}\right)$, where $m$ and $n$ are different subsuript numbers, while $m$ precedes $n$ in the ordinal series, we can express the relations already considered by taking, if we choose, $r_{1}$ as an origin, and by employing the relation of antecedent and consequent. In this case we can write, upon the basis of 85 (5), 


$$
\begin{gathered}
r_{m}<_{r_{1}} r_{m+1}, \\
F\left(e \mid r_{m} r_{m+1}\right) ; e \neq r_{m} ; e \neq r_{m+1} .
\end{gathered}
$$

Whence follows $e<_{r_{1}} \boldsymbol{r}_{m+1}$.

Furthermore : $r_{m+1}<_{r_{1}} r_{n}$ by $85(5)$,

And so, if $e=g$, we have:

$$
r_{n}<_{r_{1}} g, r_{n} \neq g \text {. }
$$

$$
g<_{r_{1}} r_{m+1}<_{r_{1}} r_{n}<{ }_{r_{1}} g .
$$

This, however, is impossible. Thus no member of any one of the new chains is equivalent to any member of any other of these new chains, or of the original chains. Since an infinite number of different pairs of non-equivalent members exist in the chain $\left(r_{1}, r_{2}, \ldots, r_{n}, \cdots\right)$, it follows that an infinite number of new chains can be constructed upon the basis of these various pairs. The process of forming such chains is itself recurrent.

In addition to the chains thus far defined, other chains of elements exist in $\Sigma$. For since, by $85, r_{1} \neq x$, it is possible to treat the pair $\left(r_{1}, x\right)$ as, in 85 , the pair $\left(r_{1}, \bar{x}\right)$ was treated. The result would be to define a chain of elements $\left(r_{1}, p_{1}, p_{2}, \cdots, p_{h}, \cdots\right)$, such that $F\left(p_{h} \mid r_{1} x\right)$, while, as before, no two elements of the chain are mutually equivalent. If any element of $\Sigma$, say $v$, is such that $F\left(v \mid r_{1} x\right)$ and $F\left(v \mid r_{1} \bar{x}\right)$, we have $F\left(v \mid r_{1} x\right)$ and $F\left(r_{1} \mid v x\right)$, and hence $v=r_{1}$. See also 72. It follows that no element of the new chain except $r_{1}$ itself is equivalent to any element of the chain $\left(r_{1}, r_{2}, \cdots, r_{n}, \cdots\right)$.

\section{General properties of resultants.}

87. A very little consideration serves to show that a new application of principle VI, to the chains of elements now defined, will lead to still further results. Before we are prepared to consider these results, we must however survey the properties of a class of elements defined in 17 . Of the infinitely numerous non-equivalent elements now known to exist in $\Sigma$, collections can be made comprising any number of elements. These may be either $E$-collections or $O$.collections. If of the former type, the collections so made can be enlarged to $O$-collections by the adjunction of suitable elements. To what laws are such adjunctions subject? This we are next to see.

88. If $\beta$ be any collection, any element $r$ such that $F(r \mid \beta)$, is a resultant of the collection $\beta$, by virtue of the definition stated in 17 . Since $r$ is thus any element such that $O(\beta \bar{r})$, and since the obverse of any element of $\beta$ may fill the place of $\bar{r}$ in this $O$-collection, it is obvious that every element of $\beta$ is also a resultant of $\beta$. Furthermore, if any element $x$ forms an $O$-collection with any partial collection of elements of $\beta, \bar{x}$ is a resultant of $\beta$. For if $O(\lambda x)$, then $O(\kappa \lambda x)$, so that if $(\kappa, \lambda)$ is the same collection as $\beta, F(\beta \mid \bar{x})$. 
89. If $F(r \mid \beta), F(t \mid \beta)$ and $F(u \mid r t)$ are all true there follows, by 63 , $F(u \mid \beta)$. Hence any mediator of a pair of resultants of a given collection is itself a resultant of the collection. By the reasoning used in 85-86, it can therefore be shown that any collection containing at least two non-equivalent elements must possess an infinite number of resultants.

90. If $\rho$ be the collection consisting of all the resultants, $r_{1}, r_{2}$, etc., of a given collection $\beta$, then any resultant of $\rho$ is also a resultant of $\beta$.

Let $q$ be such that $F(q \mid \rho)$. Then $O(\rho \bar{q})$, and hence $O(\bar{\rho} q)$. By the definition of a resultant, $\beta$ is a collection which becomes an $O$-collection if any element of $\bar{\rho}$, that is, if the obverse of any one of the resultants of $\beta$, be adjoined to $\beta$. Hence $\beta$ forms an $O$-collection in case any member of the $O$-collection $O(\bar{\rho} q)$, except $q$, is adjoined to $\beta$. By $36(5)$ it follows that $O(\beta \bar{q})$. Hence $F(\beta \mid q)$.

The collection $\rho$ is consequently a collection which contains all of its own resultants. It also contains the resultants of all the partial collections which can be found by selecting certain elements from the collection $\beta$.

91. If $y$ is an element of $\Sigma$, selected at pleasure, and if $\beta$ is any collection, and if a determinate element $c$ exists such that, whatever element $b_{n}$ of $\beta$ is chosen, $c$ is a mediator of the pair $\left(y, b_{n}\right)$, then $c$ is also a mediator of any and every pair that can be formed by combining $y$ with any resultant whatevèr, say $r_{x}$ of $\beta$. For if $r_{x}$ be any resultant of $\beta$, then $O\left(\beta \bar{r}_{x}\right)$. And if $c$ exists such that $F\left(c \mid y b_{n}\right)$ is true, whatever element $b_{n}$ of $\beta$ may be chosen, then $O\left(\bar{c} y b_{n}\right)$ is true of every member $b_{n}$ of $\beta$. Hence, by $36(5), O\left(\bar{c}_{y} r_{x}\right)$. And hence $F\left(c \mid y r_{x}\right)$.

The converse of this theorem is obvious. That is, if an element $c$ is a mediator of every pair $\left(y, v_{x}\right)$ consisting of $y$ and some resultant of $\beta, c$ is a mediator of $\left(y, b_{n}\right)$ where $b_{n}$ is any element chosen at pleasure from $\beta$. For the elements of $\beta$ are themselves amongst the resultants of $\beta$.

There always exists an element $c$ having the properties here in question, since $y$ itself is such an element. For $F(y \mid y x)$ is true of every element $x$. But, as will soon appear, there are, in certain important classes of cases, elements possessing this property which are not equivalent to the chosen element $y$.

92. If $\gamma$ be the collection of all those elements, $c_{v}$, which possess the property discussed in 91 , viz., if $\gamma$ be the collection of those elements $c_{v}$, such that $F\left(c_{v} \mid y r_{x}\right)$ is true for a determinate selected element $y$, and for every resultant, $r_{x}$, of $\beta$, then every resultant of $\gamma$ is itself a member of the collection $\gamma$.

For let $t$ be such that $F(t \mid \gamma)$, so that $O(\bar{\gamma} t)$. Since $\left(y, r_{c}\right)$ is such that $O\left(y r_{x} \bar{c}_{v}\right)$ is true of every element $\bar{c}_{v}$ of $\bar{\gamma}$, while $O(\bar{\gamma} t)$, it follows that $O\left(y r_{x} \bar{t}\right)$, by $36(5)$. Hence $F\left(t \mid y r_{x}\right)$.

If $\rho$ be the collection of all the resultants of $\beta$, and if $\gamma$ be the collection of all those elements, such as $c_{v}$, which with reference to a selected element $y$, 
have the property of being, every one of them, such that $F\left(c_{v} \mid y r_{x}\right)$ is true of every element $r_{x}$ of $\rho$, then, as now appears, the collections $\gamma$ and $\rho$ are collections each of which contains all of its own resultants.

\section{Pairs of conjugate resultants.}

93. We are now prepared to consider more minutely the consequences of principle VI. By that principle, if a collection $\succcurlyeq$ possesses any complement $w$, so that $O(\vartheta w)$, a complement $v$ also exists such that $O\left(\vartheta_{v}\right)$ while, whatever element $t_{n}$ of $\vartheta$ be selected, $O\left(v w t_{n}\right)$. Stating this requirement in terms of the $F$-collections, we have, as the conditions set forth in the hypothesis of the principle, the existence of an element $\bar{w}$, such that $F(\bar{v} \mid \vartheta)$. The consequence according to the principle is that $\bar{v}$ also exists such that $F(\bar{v} \mid \vartheta)$, while, since $O\left(v v v t_{n}\right), O\left(\bar{v} \bar{w}_{\bar{v}} \bar{t}_{n}\right)$, and therefore $F\left(t_{n} \mid \bar{v} \bar{v}\right)$ for every element $t_{n}$ of $\vartheta$. Let $\bar{w}=q$, and $\bar{v}=r$. Then principle VI asserts that whatever resultant $q$ of a collection $\vartheta$ be selected, there always exists a resultant of $\vartheta$, namely $r$, such that every element of $\vartheta$ is a mediator (that is a resultant) of the pair $(q, r)$. It readily follows that every resultant of $\vartheta$ is a mediator of $(q, r)$. For if $p_{k}$ be any resultant of $\vartheta$, then $O\left(\vartheta \bar{p}_{k}\right)$. And since $O\left(v w t_{n}\right)$ is true of every element of $\vartheta$, while $O\left(\vartheta \bar{p}_{k}\right)$, it follows, by $36(5)$, that $O\left(v w p_{k}\right)$, that is $O\left(\bar{q} \bar{v} p_{k}\right)$, and hence $F\left(p_{k} \mid q r\right)$.

94. If $q$ is any determinate resultant of $\vartheta$, and if $r$ is an element related to $q$ in the way set forth in 93 , then any element, $r^{\prime}$, such that $F\left(q r^{\prime} \mid p_{k}\right)$ is true for every resultant of $\vartheta$, i. e. any element $r^{\prime}$ such that $F\left(q r^{\prime} \mid t_{n}\right)$ is true of every element of $\vartheta$, is also such that $r^{\prime}=r$. For, since $r$ and $r^{\prime}$ are both of them resultants of $\vartheta$, we have $F\left(q r \mid r^{\prime}\right)$, because of the definition of $r$; and also $F\left(q r^{\prime} \mid r\right)$ because of the definition of $r^{\prime}$. Hence $r^{\prime}=r$ by $52(2)$. We may consequently let $r$ stand as the unique representative of the class of those resultants of $\vartheta$ which, when $q$ is given, fulfil, with respect to $q$, the requirement of principle VI. With this understanding, we shall henceforth characterize $r$ as the conjugate resultant of $q$ in, or with respect to the collection $\vartheta$. If $r$ is given instead of $q$, some equivalent of $q$ is nevertheless predetermined as a conjugate of $r$; and if $q$ be selected as the unique representative of the class of elements which are equivalent to itself, we may regard the relation of $q$ and $r$ as wholly symmetrical; and so we may henceforth speak of the pair $(q, r)$ as a pair of conjugate resultants of the collection $\vartheta$, or more briefly as a conjugate pair in, or with respect to, $\vartheta$. We shall symbolize the relation in question thus: $J(q r ; \vartheta)$. This symbol is to be read as the assertion: "The pair $(q, r)$ is a pair of conjugate resultants of the collection $\vartheta$," or " is a conjugate pair in $\vartheta$," or " with respect to $\vartheta . "$ Were $\vartheta$ a pair, as for instance $(x, y)$, we could write $J[q r ;(x, y)]$. 
95. If the collection $\vartheta$ is made to include all of the elements of $\Sigma$, the pair $(q, r)$ becomes a pair of mutually obverse elements so that $q=\bar{r}$. This appeared already in 27, when the first use of principle VI was made. If $\vartheta$ is an $O$-collection, a conjugate pair $(q, r)$ such that $O\left(\bar{q} \bar{r} t_{n}\right)$ while $O(\vartheta)$ is true, is a pair such, by principle II, that $O(\bar{q} \bar{r})$. Hence, in this case, $O(q r)$, and thus any pair that is a pair of conjugates with respect to an $O$-collection is an $O$-pair. If, in case of any collection $\vartheta$, a pair $(q, r)$, conjugate with respect to $\vartheta$ is such that $q=r$, then, since $F\left(t_{n} \mid q r\right)$, every element $t_{n}$ of $\vartheta$ is such that $t_{n}=q=r$; and therefore, in this case, a single element may be taken as the unique representative both of the whole collection $\vartheta$, and of all of its possible pairs of conjugate resultants (see $52(1)$, and 70 ).

96. In case of any pair of conjugate resultants of a system $\beta$, i. e., in case of $(q, r)$ such that $J(q r ; \beta)$, the resultants of the pair $(q, r)$, and the resultants of $\beta$, form precisely identical collections. If $\beta$ is enlarged either to an $O$-collection, or so as to include all of the elements of $\Sigma$, the entire system $\Sigma$ becomes the collection of the resultants of any one of the possible pairs of obverse elements of $\Sigma$, such, for instance as $(x, \bar{x})$; any one of these pairs being, as we now know, such that, if $O(\beta), J(x \bar{x} ; \beta)$.

97. If $\beta$ is a given collection, and if $(q, r)$ is a conjugate pair of its resultants, and if $u$ is any third resultant of $\beta$, not equivalent either to $q$ or to $r$, then the conjugate resultant $v$ of $u$ can be found by considering merely the triad $(q, r, u)$. According to principle VI there is, namely, a resultant $v$ of this triad* such that $F(v u \mid q)$ and $F(v u \mid r)$. Since $v$ is a resultant of the triad, $F(q r u \mid v)$. But meanwhile, since $J(q r ; \beta)$, and $F(\beta \mid v), F(q r \mid v)$ by the definition of a conjugate pair, so that from $F(q r u \mid v)$ the element $u$ may be stricken out. Since $v$, then, is such that $F(q r \mid v)$, while $F(v u \mid q)$ and $F(v u \mid r)$, it is easy to show that any resultant $p_{k}$ of $\beta$ is such that $F\left(p_{k} \mid v u\right)$. For $F\left(p_{k} \mid q r\right)$, since $(q, r)$ is a conjugate pair. But from $F\left(p_{k} \mid q r\right), F(q \mid u v)$ and $F(r \mid u v)$, follows, by $73, F\left(p_{k} \mid u v\right)$.

If, then, a single conjugate pair of resultants of a collection $\beta$ is given, viz., $(q, r)$, the conjugate of any third resultant of $\beta$, such as $u$, is equivalent to the conjugate of $u$ in the collection of the resultants of the triad $(q, r, u)$. If $\beta$ is an $O$-collection, or if $\beta$ includes all of the elements of $\Sigma$, the conjugate of $x$ in the triad $(x, z, \bar{z})$ is obviously $\bar{x}$.

98. If $(\beta, x)$ be any collection that includes a given element, $x$, the conjugate resultant of $x$ with respect to the collection $(\beta, x)$ is one of the resultants of $\beta$. For let $q$ be such a resultant. $F(q \mid q x)$ is in any case true of $q$. But $q$ has, in addition, to be such that $F(q \mid \beta x)$, while, whatever element $b_{n}$, of $\beta$,

\footnotetext{
* Identical with what KEMPE calls the " unsymmetrical resultant" of the triad $(q, r, u)$. KEMPE does not directly define our conjugate resultants in general, but builds his theory upon that of the resultants of triads.
} 
be selected, $F\left(q x \mid b_{n}\right)$; i. e., $O\left(\bar{q} \bar{x} b_{n}\right)$. By the adjunction of all elements of $\beta$ besides $b_{n}$, we obtain, hereupon, $O(\bar{q} \bar{x} \beta)$. But since $F(q \mid \beta x), O(\bar{q} x \beta)$. Hence $O(\bar{q} \beta)$. Hence $F(\beta \mid q)$.

99. If $\beta$ and $\alpha$ are such that $F(\beta \mid \alpha)$, then $\beta$ and $\alpha$ possess at least one common resultant. For let $a$ be an element of $\alpha$, chosen at pleasure. Adjoin $\bar{i}$ to the collection $\beta$, and consider the conjugate resultant of $\bar{a}$ in the collection $(\bar{u}, \beta)$. Let this resultant be the element $b$. By 98, $F(\beta \mid b)$. By the definition of a conjugate resultant $F\left(\bar{u} b \mid b_{n}\right)$ is true of every element $b_{n}$ of $\beta$. By hypothesis, however, $F(\beta \mid \alpha)$. Hence by $56, F(x \mid \bar{a} b)$. Since $a$ is itself an element of $\alpha$, the superfluous obverse $\bar{a}$ may be omitted, so that $F(\alpha \mid b)$. Hence the element $b$ is a resultant of $\beta$ and also of $\alpha$.

The importance of this theorem for the geometrical application of our theory (since the theorem may be called the theorem regarding intersections or transversals) justifies a proof directly in terms of $O$-collections.

If, namely, $O(\delta \epsilon)$, there exists $x$ such that $O(\delta \bar{x})$ while $O(\epsilon x)$. For let us select at pleasure any element $d$ of $\delta$. Consider the collections $(\bar{d}, \bar{\epsilon})$, and, with respect to that collection, define the conjugate resultant of $\bar{d}$ in $(\bar{d}, \bar{\epsilon})$. Let $x$ be this resultant. By $98, O(\bar{\epsilon} \bar{x})$ is true. Hence $O(\epsilon x)$. By the definition of a conjugate resultant $F\left(\bar{e}_{r} \mid x \bar{l}\right)$ is true of every element $\bar{e}_{r}$ of $\bar{\epsilon}$. Hence $O\left(e_{r} x \bar{d}\right)$ is true of every element $e_{r}$ of $\epsilon$, while $O(\delta \epsilon)$. Hence, by $24(4), O(\bar{\delta} x \bar{d}) . \quad \bar{d}$ is a repetition of some element of $\bar{\delta}$, and may be stricken out (by 37). Hence $O(\bar{\delta} x)$. Hence $O(\delta \bar{x})$, while, as above shown, $O(\epsilon x)$. So the theorem is proved.

Since this process may be repeated for every element of $\delta$ and also of $\epsilon$, the variety of elements of the type $x$, in case the elements of $\delta$ and of $\epsilon$ include nonequivalent pairs, is, in general, by principle $\mathrm{V}$, and 89 , infinite.

The relations of pairs of conjugate resultants in various collections.

100. It is frequently important to bring the various pairs of conjugate resultants which exist in different collections into relation with one another. The procedure by which this is accomplished will lead us at once to the threshold of the ordinary algebra of logic, which, as originally developed, was based upon observing certain properties of the system $\Sigma$, in cases where this system was interpreted as a collection whose elements are either classes or propositions.

Let the conjugate resultant of $y$ in the collection $(y, \beta)$ be $x$, so that $J(x y ;(\beta, y))$. Then, by $98, F(x \mid \beta)$ is true. Hereupon, if we select, amongst the resultants of $\beta$, that one, say $z$, which is the conjugate resultant, with respect to $\beta$, of the element $x$ just determined, so that, while $J(x y ;(\beta, y))$, it is also true that $J(x z ; \beta)$, then it follows that $F\left(y b_{n} \mid z\right)$ is true for every element $b_{n}$ of $\beta$. For $z$ is such that $F\left(x z \mid b_{n}\right)$, and is also such that $F(\beta \mid z)$. Since $F\left(b_{n} \mid x y\right)$ is true (by the foregoing) for every element $b_{n}$ of $\beta$, while 
$F(\beta \mid z)$, it follows, by $36(5)$ and 57 , that $F^{\prime}(x y \mid z)$; and since $F^{\prime}\left(b_{n} \mid x z\right)$, that is $F\left(b_{n} \bar{z} \mid x\right)$, while $F(x \mid z \bar{y})$, we have $F\left(b_{n} \bar{z} \mid z \bar{y}\right)$, whence follows $F\left(z \mid b_{n} y\right)$.

We have, therefore, the result, that, if $y$ be any element, and $\beta$ any collection, then, in case $x$ is such that $J(x y ;(\beta, y))$ and $z$ is such that $J(x z ; \beta)$, the two elements $x$ and $z$ constitute a conjugate pair of the resultants of $\beta$, while just this pair stands in what we may regard as an unique relation to $y$. The pair $(x, z)$ is namely such that, for the first, $z$ is a mediator between $y$ and any element $b_{n}$ of $\beta$ which may have been chosen for comparison with $y$ and with $z$. By $91, z$ is consequently also a mediator between $y$ and any resultant, $r_{r}$, of $\beta$, so that, whatever resultant $r_{v}$, of $\beta$, we may select $F\left(r_{v} y \mid z\right)$. Moreover, whatever resultant $z^{\prime}$ of $\beta$ possesses the property just ascribed to $z$, must be equivalent to $z$. For if $z^{\prime}$ exists such that $F\left(z^{\prime} \mid \beta\right)$, while $F\left(z^{\prime} \mid r_{v} y\right)$ is true, whatever resultant $r_{v}$ of $\beta$ we choose to consider, then, since $z$ itself is a resultant of $\beta, F\left(z^{\prime} \mid z y\right)$, while, by the definition of $z, F\left(z \mid z^{\prime} y\right)$, and hence $z=z^{\prime}$. Therefore $z$ may be taken as the unique representative of its own class of equivalents. Meanwhile, $x$ possesses, with reference to $\beta$ and $y$, the property of being a resultant of $\beta$ such that every element of $\beta$ is a mediator between $x$ and $y$. Consequently, since $F\left(b_{n} \mid x y\right), F\left(b_{n} \bar{y} \mid x\right)$, and hence $x$ is a mediator between $\bar{y}$ and whatever element $b_{n}$ of $\beta$ may have been chosen. By $91, x$ is accordingly a mediator between $\bar{y}$ and whatever resultant, $r_{v}$, of $\beta$, may have been chosen. Whatever resultant, $x^{\prime}$, of $\beta$, possesses the property just ascribed to $x$, is such that $x=x^{\prime}$.

101. Conjugate limits of a collection with reference to a base. We may sum up the result of the foregoing thus: If any element $y$ be chosen, at our pleasure, as what we shall now call a base, and if hereupon any collection $\beta$ be considered with reference to this base, then there exists a pair, and (barring for the moment the consideration of equivalent elements), a single pair, of conjugate resultants of $\beta$, which is so related to $y$ and to $\beta$ that (1) one of these two resultants (which we shall now symbolize by $p$ ) is such that $F\left(p \mid b_{n} y\right)$ for every element of $\beta$, and $F\left(p \mid r_{v} y\right)$ for every resultant of $\beta$; while $(2)$ the other of these resultants, which we shall now symbolize by $s$, is such that $F\left(b_{n} \mid y s\right)$, and $F\left(r_{v} \mid y s\right)$, i. e., such that $F\left(b_{n} \bar{y} \mid s\right)$, and $F\left(r_{v} \bar{y} \mid s\right)$. If $\gamma$ be the collection of the totality of those elements of $\Sigma$ which are mediators between $y$ on the one hand and each and every element and resultant of $\beta$, separately considered, on the other hand (see 92), then $p$ has the property of belonging at once to the collection $\gamma$, and to the collection $\rho$; where $\rho$ is, as before, the collection of all the resultants of $\beta$. Any element possessing the property of belonging at once to $\gamma$ and to $\rho$, is equivalent to $p$, which may therefore be viewed, for present purposes, as the unique representative of its own class of equivalent elements. Barring equivalent elements, then, the collections $\gamma$ and $\rho$ have only this element $p$ in common. If $\gamma^{\prime}$ be the collection of 
those elements of $\Sigma^{\prime}$ which are mediators between $\bar{y}$ on the one hand, and each and every resultant of $\beta$, separately considered, on the other hand, then $s$ has the property of belonging at once to the collection $\gamma^{\prime}$, and to the collection $\rho$. Any element possessing the property of belonging at once to $\gamma^{\prime}$ and to $\rho$, is equivalent to $s$, which may therefore be viewed, for the purposes of forming $O$-collections and $F$-collections, as the unique representative of its own class of equivalent elements. Barring equivalent elements, then, the collections $\boldsymbol{\gamma}^{\prime}$ and $\rho$ have only the element $s$ in common. Of the pair $(p, s)$, each resultant of $\beta$ is a mediator. If, for the collection $\beta$, and for $\rho$, the collection of the totality of the resultants of $\beta$, the pair $(p, s)$ alone is substituted, and if hereupon this pair is treated precisely as, in the foregoing, $\beta$ itself has been treated, that is, if the resultants of $(p, s)$ are first defined, and then their collection, viz., $\rho$, is compared with $y$, the same pair $(p, s)$, is once more found as that pair of conjugate resultants of the collection $(p, s)$ itself, whose relation to $y$ is the relation heretofore characterized.

We shall now call the pair $(p, s)$ a pair of conjugate limits of $\beta$ with reference to the buse $y$. For a given collection $\beta$, and for a given base $y$, principle VI thus requires us to define one such pair, and (barring equivalent eléments), but a single pair, viz., $(p, s)$, which may be viewed as the pair of conjugate limits in question. This pair, being a pair of mutually conjugate resultants of $\beta$, is symmetrically disposed with reference to the collection of the resultants of $\beta$. But, as has appeared in the foregoing, the pair $(p, s)$ is not, in general, symmetrically disposed with respect to the enlarged collection $(\beta, y)$. For $p$ is a mediator between $y$ and each resultant of $\beta$ separately considered; while each resultant of $\beta$, separately considered, is a mediator of the pair $(y, s)$. To mark this difference of relative position of $p$ and $s$ we may call : $p$ the inferior limit of $\beta$ with respect to $y ; s$ the superior limit of $\beta$ with respect to $y$; while $y$ is the base of this pair of conjugate limits of $\beta$. It is at once obvious that if we choose $\bar{y}$ as base instead of $y, s$ would become the inferior, and $p$ the superior limit of $\beta$ with respect to $\bar{y}$.

102. We are now in a position to extend our result from the case of a single collection $\beta$, to the case of a set of collections $\beta, \gamma, \delta$, etc., and in fact to the set of all possible collections of the elements of $\Sigma$. Holding a given base, $y$, chosen at pleasure, constant, we may consider any and all collections of the elements of $\Sigma$ with reference to this one chosen base. If we do so, then, whatever collection, $\alpha, \beta, \gamma, \delta$, etc., we select, we shall find, by a process wholly analogous to the foregoing, that there exists an unique pair of resultants of any one such collection, such that this pair is, for that collection, the pair of conjugate limits of the collection with respect to $y$. Of this pair, one element is the superior, and the one the inferior limit of the collection in question, with respect to this chosen and constant base, while the choice of the base is arbitrary. The 
limits are each time functions of the collection in question, and may be regarded as functions of that alone, so long as the base is held constant. *

If, while the base itself remains constant, a collection is altered, by the adjunction, or by the omission of elements, its limits alter or remain invariant, in ways whose laws are now to be defined. If various collections are considered with reference to the same base, their respective superior or inferior limits may be considered as new collections, with results whose laws are also to be determined. But if, instead, while any collection or sets of collections remain constant, the base is altered, so that an element $y^{\prime}$ or $y^{\prime \prime}$ takes the place of $y$, then the pairs of conjugate limits of any given collection with reference to the new base remain invariant, or alter, in accordance with still other principles (which we shall consider in chapter VI). We shall discuss these various cases in order. But in the rest of the present chapter, the base shall remain constant.

103. Adjunction of the base to a collection. If, to the collection $\beta$, while the base remains constant, the base $y$ is itself adjoined, the inferior limit of $(\beta, y)$ becomes equivalent to $y$ itself ; but the superior limit remains invariant. This appears from the reasoning used in 100.

104. Adjunction of the obverse of the base; adjunction of resultants; other cases. If the base $y$ is held constant, and if $\bar{y}$ is thereupon adjoined to $\beta$, the inferior limit remains constant, while the superior limit of $(\beta, \bar{y})$ becomes equivalent to $\bar{y}$. The reasoning used in 100 can be employed to prove this also. - If any resultant of $\beta$ is adjoined to $\beta$, or if any collection of the resultants of $\beta$ is adjoined to $\beta$; the superior and inferior limits of the enlarged collection, so long as the base $y$ is held constant, remain invariant; as appears from the reasoning used in 91,92 . Hence, by 89 , if to a given collection, any mediator of any pair of its elements or of its resultants is adjoined, the limits remain constant; as they also do if the elements of a collection are repeated any multitude of times. If $y$ is the constant base, and if any element or collection of elements of the collection $\gamma$, of 92 and 101, be adjoined to $\beta$, then, while the inferior limit, in general, alters, the superior limit of the collection remains in so far invariant. If any element or collection of elements chosen from the collection $\gamma^{\prime}$ of 101 be adjoined to $\beta$, this adjunction leaves the inferior limit of the enlarged collection invariant, while altering, in general, the superior limit.

Elements may be omitted from collections in a manner which is subject to these same laws. Thus, the omission of such repetitions of elements as occur in a collection does not alter either the superior or the inferior limits, etc.

* The conception that the elements usually known as the products and sums of the algebra of logic are relative to a chosen constant base (the zero of the usual algebra of logic), and that the choice of what element of $\Sigma$ shall be treated as the zero-element is essentially arbitrary, is KEMPE's. But KEMPE develops this conception solely on the basis of his theory of the symmetrical and unsynmetrical resultants of triads. By the more general concept of the conjugate resultants of collections, I have generalized KENPE's theory so as to be able to apply it, from the start, to collections of any multitude whatever. 
The operation of determining the superior and inferior limits of a collection is obviously independent of the order in which the elements are arranged or considered, and may consequently be called a commutative operation.

105. The transformation of a collection into its obverse collection. If a collection $\beta$ be transformed into its obverse collection (the base $y$ remaining constant), if $p$ be the inferior, and $s$ be the superior limit of $\beta$, and if $p^{\prime}$ be the inferior and $s^{\prime}$ be the superior limit of $\bar{\beta}$ then the two equivalences hold good:

$$
\bar{p}=s^{\prime} ; \text { and } \bar{s}=p^{\prime} \text {. }
$$

For, by the definition of an inferior limit, $F^{\gamma}\left(p \mid y r_{v}\right)$ for every resultant, $r_{v}$. of $\beta$, while $F(p \mid \beta)$. Whence there follows (1) $F\left(\bar{p} \mid \bar{y} \bar{r}_{v}\right)$ for every resultant $\bar{r}_{v}$ of $\bar{\beta}$; while $(2) F(\bar{p} \mid \bar{\beta})$. From (2) it follows that $\bar{p}$ is a determinate resultant of $\bar{\beta}$. From (1) follows, by transfer, $F\left(\bar{p} y \mid \bar{r}_{v}\right)$, whatever resultant $\bar{r}_{v}$ of $\bar{\beta}$ may be chosen. Thus $\bar{p}$ is such a resultant of $\bar{\beta}$ that whatever element or resultant $\bar{r}_{v}$ of $\bar{\beta}$ may be chosen, this element or resultant of $\bar{\beta}$ is a mediator between $\bar{p}$ and $y$. Hence $\bar{p}$ is equivalent to the superior limit of $\vec{\beta}$ with respect to the base $y$. Hence $\boldsymbol{s}^{\prime}=\bar{p}$.

Furthermore, since $s$ is the superior limit of $\beta$ with respect to $y$, we have $F\left(\dot{r}_{v} \mid s y\right)$, whatever resultant, $r_{r}$, of $\beta$ we may choose; while, at the same time $F(s \mid \beta)$. It follows that $F(\bar{s} \mid \bar{\beta})$, so that $\bar{s}$ is a determinate resultant of $\bar{\beta}$; while $F\left(\bar{r}_{v} \mid \bar{s} \bar{y}\right)$, i. e., $F\left(\bar{r}_{v} y \mid \bar{s}\right)$, for every resultant $\bar{r}_{v}$ of $\bar{\beta}$; so that $\bar{s}$ is a mediator between $y$ on the one hand, and each and every $\bar{r}_{v}$ of $\bar{\beta}$ on the other hand. Thus $\bar{s}$ is equivalent to the inferior limit of $\bar{\beta}$ for the base $y$. The equivalences in question, viz., $\bar{p}=s^{\prime}$; and $\bar{s}=p^{\prime}$, are accordingly proved.

106. The combination of collections. The associative law in the determination of pairs of conjugate limits with respect to a constant base. If we consider the inferior limit of a collection $\beta_{1}$, with respect to a base $y$, and also the inferior limits, with respect to the same base, of collections $\beta_{2}, \beta_{3}, \cdots, \beta_{n}, \ldots$, in any multitude of collections, and if $\pi$ be the collection of all these inferior limits, while $\omega$ is the collection consisting of the totality of collections $\left(\beta_{1}, \beta_{2}, \ldots, \beta_{n}, \ldots\right)$, then the inferior limit of $\pi$ is equivalent to the inferior limit of $\omega$ (the subscripts $1,2, \ldots, n, \ldots$ are now no longer to be viewed as ordinal numbers, but merely as distinguishing marks).

The inferior limit $p_{\omega}$ of the collection $\omega$ is, in fact, an element such that $F\left(\omega \mid p_{\omega}\right)$, while if $b_{n}^{(k)}$ is any element of any collection $\beta_{n}$ in the set $\left(\beta_{1}, \beta_{2}, \cdots, \beta_{n}, \cdots\right), F\left(p_{\omega} \mid y b_{n}^{(k)}\right)$. Meanwhile, if $p_{n}$ is the inferior limit of any collection $\beta_{n}$, then $F\left(\beta_{n} \mid p_{n}\right)$, while $F\left(p_{n} \mid b_{n}^{(k)} y\right)$ for every element $b_{n}^{(k)}$ of $\beta_{n}$. If $\pi$ is the collection $\left(p_{1}, p_{2}, \cdots, p_{n}, \cdots\right)$, then its inferior limit $p_{\pi}$ is an element such that $F\left(\pi \mid p_{\pi}\right)$, while, whatever $p_{n}$ may be selected, $F\left(p_{\pi} \mid p_{n} y\right)$. Since $F\left(p_{n} \mid b_{n}^{(k)} y\right)$, and $F\left(p_{\pi} \mid p_{n} y\right)$, it follows by 71 , that $F\left(p_{\pi} \mid b_{n}^{(k)} y\right)$; and this latter assertion holds true, whatever element $b_{n}^{(k)}$ of any collection $\beta_{n}$ 
may be selected, that is, whatever element of $\omega$ may be selected. Moreover, whatever element $p_{n}$ of $\pi$ may be selected, since $\nu_{n}$ is a resultant of $\beta_{i:}, \nu_{n}$ is also a resultant of $\omega(88)$. Thus $F\left(\omega \mid p_{n}\right)$, whatever element $\nu_{n}$ of $\pi$ may be selected, while $F\left(\pi \mid p_{\pi}\right)$. Hence (by 57$), F\left(\omega \mid p_{\pi}\right)$. Thus $p_{\pi}$ is a resultant of $\omega$, while $p_{\pi}$ is such that $F\left(p_{\pi} \mid b_{n}^{(h)} y\right)$ for every element $b_{n}^{\prime \prime)}$ of $\omega$. Hence $p_{\pi}=p_{\omega}$.

The operation of determining the inferior limit of a collection $\omega$ which is composed of a set of collections $\left(\beta_{1}, \beta_{2}, \ldots, \beta_{n}, \ldots\right)$, is consequently associ(1tive with respect to the operation of separately determining the inferior limit of each one of these collections $\left(\beta_{1}, \beta_{2}, \cdots, \beta_{n}, \cdots\right)$.

By a precisely similar reasoning, one can obviously prove that the operation of determining the superior limit, $s_{\omega}$, of the collection $\omega$, is associative, in case we separately determine the superior limits of $\beta_{1}, \beta_{2}$, etc., and use such determination as the basis for determining $s_{\omega}$.

107. Cross collections. If a set of collections $\left(\beta_{1}, \beta_{2}, \ldots, \beta_{n}, \ldots\right)$ is given (where the multitude of collections in question is wholly unrestricted), and if a collection $\lambda$ is formed by selecting, at pleasure, one element, and one only (say $b_{1}^{(i)}$ ), from $\beta_{1}$, one element, and one only (say $b_{2}^{(j)}$ ), from $\beta_{2}$, and one element, and only one, from each of the collections of the set (so that, for instance, $b_{n}^{(c)}$ is selected from $\left.\beta_{n}\right)$, then the collection $\lambda_{z}$, that is, the collection $\left(b_{1}^{(i)}, b_{2}^{(j)}, \ldots, b_{n}^{(n)}, \ldots\right)$ shall be called a cross-collection of the set $\left(\beta_{1}, \beta_{2}, \ldots, \beta_{n}, \ldots\right)$. As many distinct cross-collections $\left(\lambda_{l}, \ldots, \lambda_{x}, \ldots, \lambda_{z}, \ldots\right)$ exist as there are distinct possible combinations of elements selected one from each of the collections of the set $\left(\beta_{1}, \beta_{2}, \cdots, \beta_{n}, \cdots\right)$. Choose now a constant base $y$, to be retained throughout what follows in this and in the next section. Hereupon, let $s_{n}$ be the superior limit with respect to $y$ of the collection $\beta_{n}$ of the original set. Let $\left(s_{1}, s_{2}, \ldots, s_{n}, \ldots\right)$ be the collection of all such superior limits, with respect to $y$, of $\beta_{1}, \beta_{2}$, etc. Let the collection $\left(s_{1}, s_{2}, \ldots, s_{n}, \ldots\right)$ be here called the collection $\vartheta$. Let the inferior limit of $\vartheta$, with respect to the base $y$, be symbolized by $p_{z}$. Next, let $p_{z}$ be the inferior limit of $\lambda_{z}$, that is, of the collection $\left(b_{1}^{(i)}, b_{2}^{(j)}, \cdots, b_{n}^{(r)}, \cdots\right)$. Let $\left(p_{t}, \cdots, p_{x}, \cdots, p_{z}, \cdots\right)$ be the collection comprising all such inferior limits of the possible cross-collections $\left(\lambda_{t}, \cdots, \lambda_{x}, \cdots, \lambda_{z}, \cdots\right)$. Let the collection $\left(p_{t}, \cdots, p_{x}, \cdots, p_{z}, \cdots\right)$ be called the collection $\psi$. Let the superior limit of $\psi$ be symbolized by $s_{\psi}$. Then we shall next inquire how $\mu_{\vartheta}$ is related to $s_{\psi}$, that is, how the inferior limit of the collection of all the respective superior limits of a given set of collections, is related to the superior limit of the collection of all the respective inferior limits of the corresponding cross-collections. To ask this question is to inquire (in the most general form possible) whether the operation of seeking inferior limits is distributive with reference to the operation of seeking the superior limits of given collections.

108. The Distributive Lan: Using the conventions of the previous section, 
regarding the cross-collections, and retaining the symbols used, we are able to assert that

$$
P_{\diamond}=s_{\downarrow} .
$$

For, by the definition of an inferior limit, whatever element $p_{z}$ we may select from the collection $\psi$, the element $p_{z}$ is such that $F\left(p_{z} \mid y b_{n}^{(r)}\right)$ for every element $l_{n}^{(r)}$ which belongs to the cross-collection $\lambda_{z}$. And, by the rule according to which this collection $\lambda_{z}$ has been formed, there exists, in each of the original collections $\beta_{n}$, an element, $b_{n}^{(r)}$, for which the foregoing assertion is true. Meanwhile, if we consider the relation of the element $b_{n}^{(r)}$ to its own collection $\beta_{n}$, of the original set $\left(\beta_{1}, \beta_{2}, \cdots, \beta_{n}, \cdots\right)$, it follows, by the definition of a superior limit, that $F\left(b_{n}^{(c)} \mid s_{n} y\right)$. Since, then, the element $b_{n}^{(c)}$ is such that $F\left(p_{z} \mid y b_{n}^{(n)}\right)$, that is, such that $F\left(b_{n}^{(r)} \mid p_{z} \bar{y}\right)$, while $F\left(b_{n}^{(r)} \mid s_{n} y\right)$, we have, by the usual rule for the elimination of an element, $F^{\prime}\left(s_{n} y \mid p_{z} \bar{y}\right)$; whence follows $F\left(p_{z} \mid s_{n} y\right)$. Since, whatever $p_{z}$ may be in question, that element of $\lambda_{z}$ which $\lambda_{z}$ has in common with any given collection $\beta_{n}$ may thus be eliminated, it follows that the relation $F\left(p_{z} \mid s_{n} y\right)$ holds of every element $l_{z}$ of the collection $\psi$, when the relation of $p_{z}$ to any element, whatever $s_{n}$, of $\theta$, is considered. Since, however, by the definition of a superior limit, $F\left(s_{\psi} \mid \psi\right)$ is true, while $F\left(p_{z} \mid s_{n} y\right)$ holds true of each element, $p_{\tilde{z}}$, of $\psi$, separately considered, in its relation to each element of $\vartheta$, separately considered, it follows, by 57 , that, whatever $s_{n}$ be chosen, the pair $\left(s_{n}, y\right)$ is such that $(1) F\left(s_{n} y \mid s_{\psi}\right)$.

Furthermore, since, by the definition of a superior limit, whatever $p_{z}$ we may select $F\left(p_{z} \mid s_{\psi} y\right)$, while, by the definition of an inferior limit, $F\left(p_{z} \mid \lambda_{z}\right)$, it follows that, whatever $\lambda_{z}$ we may select, $F\left(\lambda_{z} \mid s_{\psi} y\right)$ is always true. Since this latter relation is general, and holds for every collection $\lambda_{z}$, without exception, we have, for every possible cross-collection, $F\left(b_{1}^{(i)} b_{2}^{(j)} \cdots b_{n}^{(r)} \cdots \mid s_{\psi} y\right)$; an assertion which remains invariant and true if, instead of selecting, from any one of the collections $\left(\beta_{1}, \beta_{2} \ldots \beta_{n} \cdots\right)$ the element which here appears in the collection $\lambda_{z}$, we select instead any other of the elements of that same collection $\beta_{1}, \beta_{2}$, ete. Consider, hereupon, once more, the element $b_{n}^{(r)}$, here selected from the collection $\beta_{n}$. Let $\lambda_{z}^{(\prime \prime)}$ be what the collection $\lambda_{z}$ becomes if that element in $\lambda_{z}$ which is derived from the collection $\beta_{n}$ is omitted. In other words, let $\lambda_{z}$ be so subjectel to partition that $\lambda_{z}$ is the same collection as $\left(\lambda_{z}^{(n)}, b_{n}^{(n)}\right)$. Then we have, from the foregoing, $F\left(\lambda_{z}^{(n)} b_{n}^{(c)} \mid s_{\psi} y\right)$, that is, by transfer, $F\left(b_{n}^{(c)} \mid s_{\psi} y \bar{\lambda}_{z}^{(n)}\right)$; while this relation holds true whatever element $b_{n}^{(v)}$ is selected from $\beta_{n}$. Since, however, $s_{n}$ is such that $F\left(s_{n} \mid \beta_{n}\right)$, while for $b_{n}^{(r)}$ any element whatever of $\beta_{n}$ may be substituted, we have, by 57 , the collection $\left(s_{\psi}, y, \bar{\lambda}_{z}^{(\prime)}\right)$ such that $F^{\prime}\left(s_{n} \mid s_{\psi} y \bar{\lambda}_{z}^{(\prime \prime)}\right)$; so that, by transfer, $F\left(\lambda_{z}^{(\prime \prime)} s_{n} \mid s_{\psi} y\right)$. It follows that, while, as before, $F\left(\lambda_{z} \mid s_{\psi} y\right)$ is true, this relation remains invariant if we substitute for any element $b_{n}^{(r)}$, of $\lambda_{s}$, the corresponding superior limit, $s_{n}$, of that collection $\beta_{n}$, from which $b_{n}^{(r)}$ has 
been selected. Such substitution may be accomplished in case of any element $b_{1}^{(i)}, b_{2}^{(j)}$, etc., independently of whether such a substitution has been made in case of any other element of $\lambda_{z}$.

For since both the assertions $F\left(\lambda_{z} \mid s_{\psi} y\right)$, and $F\left(\lambda_{z}^{(n)} s_{m} \mid s_{\psi} y\right)$, hold good in case an element $b_{m}^{(q)}$ of $\lambda_{z}$ is selected from any collection $\beta_{m}$ which is not the collection $\beta_{n}$, we could, by a repetition of the foregoing process of reasoning, show that the relation expressed in $F\left(\lambda_{z} \mid s_{\psi} y\right)$ not only remains invariant whichever one of the two elements, $b_{n}^{(v)}$ or $b_{m}^{(q)}$ is selected as that element for which the corresponding $s_{n}$ or $s_{m}$ is substituted; but also remains invariant when for both of them, simultaneously, the corresponding superior limits of $\beta_{n}$ and $\beta_{m}$ are substituted. This result can be extended, at pleasure, therefore, to any number, or to any partial collection, or to all of the elements of $\lambda_{z}$, without regard to their multitude. The relation $F\left(\lambda_{z} \mid s_{\psi} y\right)$ therefore remains invariant in case, for each and every member of $\lambda_{z}$, we substitute the corresponding element $s_{n}$, viz., the superior limit of that collection $\beta_{n}$ from which the member of $\lambda_{z}$ which is each time in question was itself selected. Hence carrying out this substitution, we have (2) $F\left(\vartheta \mid s_{\psi} y\right)$.

But we have seen above, by (1), that $F\left(s_{n} y \mid s_{\psi}\right)$ is true. By adjunction it follows from this that $F\left(\vartheta y \mid s_{\psi}\right)$ is true. Hence we have at once true the two assertions (2) $F\left(y \mid \vartheta \bar{s}_{\psi}\right)$ and $F\left(y \mid \bar{\vartheta}_{s_{\psi}}\right)$. Hence $F\left(\vartheta \bar{s}_{\psi} \mid \bar{\vartheta}_{s_{\psi}}\right)$. Hence (3) $F\left(\vartheta \mid s_{\psi}\right)$, by 54 .

By (1), therefore, $F\left(s_{n} y \mid s_{\psi}\right)$ is true, whatever element $s_{n}$ of $\vartheta$ we may choose ; while, by (3), $F\left(s_{\psi} \mid \vartheta\right)$, so that $s_{\psi}$ is a resultant of $\vartheta$. It follows that $s_{\psi}$ is an element such that it is a mediator betwenn $y$ and every element of $\vartheta$ separately considered while $s_{\psi}$ is also a resultant of $\vartheta$. Hence, by the definition of an inferior limit, $s_{\downarrow}=p_{\vartheta}$; and the theorem is proved.*

109. The second form of the distributive law. Still retaining constant the base $y$, let $s_{z}$ be the superior limit of any cross-collection $\lambda_{z}$, as such collections were defined in 107 . Let the collection of all the superior limits of the cross-

* The demonstration of the distributive law here given may be regarded as a generalization of KEMPE's treatment of the symmetrical resultants of triads; although this generalization involves considerations which are somewhat peculiar to the present form of the theory of conjugate resultants. When the relations of the $F$-collections are regarded as degenerating into the specialized but more familiar relation of antecedent and consequent, the proof of the distributive law becomes subject to those difficulties whose treatment by Mr. C. S. PErrce, by Schrokder, and by Dr. Huntington, are summed up by Dr. Huntington in his Sets of Postulates for the Algebra of Logic (these Transactions, Jaly, 1904).. The difficulties in question are a test of the sort and of the amount of information which is surrendered when, instead of the $F$-relations viewed, so to epeak, in their entirety, we confine ourselves to relations which are defined, for all the collections concerned, merely with reference to a common origin, and when we thereupon define the usual logical "sums" and "products" solely npon the basis of the antecedent-consequent relations. KEMPE's Theory, like the usual one, extends the distributive law by induction from pairs of triads to any number of cases. The present treatment, in this paper, applies the distribative law at once to collections of any multitude whatever. 
collections $\left(s_{t}, \ldots, s_{x}, \cdots, s_{z}, \ldots\right)$, be called the collection $\phi$. Let the inferior limit of $\phi$ be $p_{\phi}$. Let the inferior limit of any collection $\beta_{n}$ be $p_{n}$. Let the collection $\left(p_{1}, p_{2}, \cdots, p_{n}, \cdots\right)$ of all such inferior limits be the collection $\epsilon$. Let the superior limit of $\epsilon$ be $s_{\epsilon}$. Then

$$
p_{\phi}=s_{\epsilon} \text {. }
$$

For whatever element $b_{n}^{(r)}$ we may select from the cross-collection $\lambda_{z}$, $F\left(s_{z} y \mid b_{n}^{(r)}\right)$ is true. And whatever collection $\beta_{n}$ we may select, and whatever $\lambda_{z}$ is in question, there exists in $\beta_{n}$ an element for which the assertion $F\left(s_{z} y \mid b_{n}^{(r)}\right)$ is true. But, in case of the collection $\beta_{n}$, the assertion $F\left(y b_{n}^{(r)} \mid p_{n}\right)$ is true. Hence, since $F\left(b_{n}^{(r)} \mid p_{n} \bar{y}\right)$ and $F\left(b_{n}^{(r)} \mid s_{z} y\right)$, it follows that $F\left(s_{z} y \mid p_{n}\right)$; an assertion which is true of every $p_{n}$ in its relation to every $s_{z}$. From $F\left(s_{z} y \mid p_{n}\right)$, follows $F\left(s_{z} \mid \bar{y} p_{n}\right)$, an assertion which is true of every element $s_{z}$ of the collection $\phi$, while $F\left(\phi \mid p_{\phi}\right)$. Hence the collection $\left(\bar{y}, p_{n}\right)$ is such that $F\left(\bar{y} p_{n} \mid p_{\phi}\right)$, or (1) $F\left(p_{n} \mid p_{\phi} y\right)$; an assertion which again holds true whatever $p_{\text {" }}$ of $\epsilon$ may be selected.

Furthermore, since $F\left(p_{\phi} \mid y s_{z}\right)$, whatever $s_{z}$ may be selected, while, by the definition of $s_{z}, F\left(s_{z} \mid \lambda_{z}\right)$, we obtain, by the usual elimination process, $F\left(p_{\phi} \bar{y} \mid \lambda_{z}\right)$ or $F\left(p_{\phi} \bar{y} \mid b_{1}^{(i)} b_{2}^{(j)} \cdots b_{n}^{(i)} \cdots\right)$. This relation remains invariant whatever element of $\beta_{1}$ be substituted for $b_{1}^{(i)}$; whatever element of $\beta_{2}$ be substituted for $b_{2}^{(j)}$; whatever element of $\beta_{n}$ be substituted for $b_{n}^{(v)}$; and so on. If, as in $108, \lambda_{z}^{(n)}$ be what $\lambda_{z}$ becomes when $b_{n}^{(r)}$ is omitted, we have, by transfer of the entire collection $\lambda_{z}^{(n)}, F\left(p_{\phi} y \bar{\lambda}_{s}^{(n)} \mid b_{n}^{(r)}\right)$; and this holds true for every element $b_{n}^{(v)}$ of $\beta_{n}$, separately considered. But $F\left(p_{n} \mid \beta_{n}\right)$. Hence the collection $\left(p_{\phi}, \bar{y}, \bar{\lambda}_{z}^{(n)}\right)$ forms an $F$-collection with every element of $\beta_{n}$, separately considered, while $F\left(p_{n} \mid \beta_{n}\right)$; and hence $F\left(p_{\phi} \bar{y} \bar{\lambda}_{z}^{(n)} \mid p_{n}\right)$; that is, $F\left(p_{\phi} \bar{y} \mid \lambda_{z}^{(n)} p_{n}\right)$. And thus for any element $b_{1}^{(i)}, b_{2}^{(j)}, b_{m}^{(\prime)}, b_{n}^{(r)}$, of $\lambda_{z}$, there may be substituted, either simultaneously with or independently of, any of the other elements, the inferior limit, $p_{1}, p_{2}, p_{m}, p_{n}$ of the collection $\beta_{1}, \beta_{2}, \beta_{m}, \beta_{n}$, from which the olement in question is selected. If the substitution is effected simultaneously for all the elements of $\lambda_{z}$, it follows that $F\left(p_{\phi} \bar{y} \mid p_{1} p_{2} \cdots p_{n} \cdots\right)$; that is, by transfer (2) $F\left(p_{\phi} \bar{\epsilon} \mid y\right)$.

But by (1) $F\left(p_{n} \mid p_{\phi} y\right)$. By the adjunction of all the elements of $\epsilon$ besides $p_{n}$ this becomes $F\left(\epsilon \mid p_{\phi} y\right)$; that is $F\left(\epsilon \bar{p}_{\phi} \mid y\right)$. By the elimination of $y$ it follows, from (1) and (2), that $F\left(\bar{\epsilon}_{\phi} \mid p_{\phi} \bar{\epsilon}\right)$. Hence $F\left(\epsilon \mid p_{\phi}\right)$. The element $p_{\phi}$ is so related to $\epsilon$ that $p_{\phi}$ is a resultant of $\epsilon$, while, by (1), whatever element $p_{n}$ of $\epsilon$ be selected, $F\left(p_{n} \mid p_{\phi} y\right)$. Hence $p_{\phi}$ is equivalent to the superior limit of $\epsilon$, and :

as was to be proved.

$$
p_{\phi}=s_{\epsilon},
$$

Thus the operation of seeking the superior limits is distributive with reference to the operation of seeking the inferior limits of given collections. 
110. The results of 91-109 may now be summarized in the somewhat less general, but (by virtue of some of our best established mental habits) more easily apprehended form of a series of statements concerning the antecedents and consequents of the members of one or more collections.

Let an element $y$ be chosen, and held constant, both as the base and as the origin with reference to which antecedents and consequents are to be determined. What we have shown is :

111. That if a given collection $\beta$ be considered, then, whatever element $c$ is an antecedent, with respect to $y$, of every element of $\beta$, separately considered, is also an antecedent, with respect to $y$, of every resultant of $\beta$ (see 91). Moreover (by 101 ; see what is there said concerning the collection $\gamma^{\prime}$ ), whatever element $c^{\prime}$ is a consequent of every element of $\beta$, is also a consequent of every resultant of $\beta$. If we consider : (I) the collection $\gamma$, consisting of all elements $c$, each of which is an antecedent of every resultant of $\beta$; (II) the collection $\rho$, consisting of all the resultants and of all the elements of $\beta$; and (III) the collection $\gamma^{\prime}$, consisting of all the elements $c^{\prime}$, each of which is a consequent of every element of $\beta$ : then each of these three collections is a collection which includes all of its own resultants. Each therefore is an internally complete or "perfect" collection.

112. If $\beta$ includes $y$, or an element equivalent to $y$, then the collection $\gamma$ reduces to the single element $y$ itself. If $\beta$ includes $\bar{y}$, or any element equivalent to $\bar{y}$, then the collection $\gamma^{\prime}$ reduces to $\bar{y}$. If $\beta$ is an $O$-collection, the same result obtains for both $\gamma$ and $\bar{\gamma}$; that is $\gamma$ reduces to $y$ and $\gamma^{\prime}$ to $\bar{y}$ (95), all the equivalents of an element being here regarded as represented by that element.

113. The collections $\gamma$ and $\rho$ have an element in common (101). This element is what we have called $p$, the inferior limit of $\beta$ with respect to $y$. Whatever element $\gamma$ and $\rho$ have in common is equivalent to $p$. The collections $\rho$ and $\gamma^{\prime}$ have an element in common, viz., $s$, the superior limit of $\beta$ with respect to $y$. Whatever element $\gamma^{\prime}$ and $\rho$ have in common is equivalent to $s$. If all the elements of $\beta$ are mutually equivalent, for instance, if they are all equivalent to $b$, then $b=p=s$. Otherwise, $p \neq s$. The elements $p$ and $s$ remain invariant in case elements of $\beta$ are repeated, any multitude of times, or in case any resultants of $\beta$ are adjoined to $\beta$. Morecver $p$ remains invariant whatever elements of $\gamma^{\prime}$ are adjoined to $\beta$; and $s$ remains invariant, whatever elements of $\gamma$ are adjoined to $\beta(103,104)$.

114. The element $p$ is definable as an element which is : (I) An antecedent of every element (and so of every resultant) of $\beta$; and (II) an element such that, whatever element of $\Sigma$ is an antecedent of every element of $\beta$, is also an antecedent of $p$. Or again, $p$ may be defined as that antecedent, of every resultant of $\beta$, which is also itself a resultant of $\beta$. Or finally, $p$ may be defined as that antecedent of every element of $\beta$ which is itself a consequent of every element $c$ which agrees with $p$ in being an antecedent of every element of . 
115. The element $s$ is definable as an element which is: (I) A consequent of every element (and so of every resultant) of $\beta$; and (II) an element such that, whatever element of $\Sigma$ is a consequent of every element of $\beta$, is also a consequent of $s$. Or again, $s$ may be defined as that consequent, of every resultant of $\beta$, which is also itself a resultant of $\beta$. Or, finally, $s$ may be defined as that consequent of every element of $\beta$ which is itself an antecedent of every element $c^{\prime}$ which agrees with $s$ in being a consequent of every element of $\beta$.

116. If $\beta$ is an $E$-collection, no one of whose elements is equivalent to $y$, and no one of whose elements is equivalent to $\bar{y}$, while $\beta$ itself contains at least one pair of non-equivalent elements, then, by 89 , and by the reasoning of chapter V, the three collections, $\gamma, \rho$ and $\gamma^{\prime}$, contain, each of them, an infinite number of elements.

117. Of the various collections here in question a principle holds true which is statable in general, on the basis of the foregoing, as a consequence of principle VI, and as holding throughout the system $\Sigma$ : If two collections are such that one of them (say $\gamma$ ) includes all those elements of $\Sigma$ each of which is an antecedent with respect to a given origin $y$, of every element of the other collection (say $\beta$ ), then there exists in $\Sigma$ an element (the inferior limit, $p$, of $\beta$, with respect to $y$ ), which is at once a member of the collection $\gamma$, and also a resultant of $\beta$. Or again: If two collections $x$ and $\beta$ exist, such that every element of $\alpha$ is an antecedent, with respect to $y$, of every element of $\beta$, then there exists at least one element of $\Sigma$ which is a consequent of every elenent of $x$, and which is also an antecedent of every element of $\beta$. The superior limit of $x$ with respect to $y$, and the inferior limit of $\beta$ with respect to $y$, both of them stand in this position. If they are not mutually equivalent, all the elements which are their mediators agree in possessing the character in question. Another and more restricted form of the same principle runs thus: Whatever infinite sequence $\kappa$, consisting of elements of $\Sigma$, is so definable that, with reference to a chosen origin, every element $k_{\text {r }}$ of the sequence possesses a consequent $k$, which also belongs to the sequence, there also exists in $\Sigma$ an element which is a consequent of every element of $\kappa$. For $\kappa$ is a collection of elements of $\Sigma$, and consequently possesses, by the foregoing, a superior limit, with respect to $y$, which also belongs to $\Sigma$. The chains of elements, defined in chapter $V$, consequently all of them possess superior limits belonging to $\Sigma . *$

118. It is now possible, without further difficulty, to point ont that the elements of $\Sigma$ possess the properties of a system of logical classes, or of entities to which the ordinary algebra of logic applies. Let the arbitrarily assumed origin

* The definite relation thus brought out between the conceptions of logical products and sums, and the conception of limits, is, so far as I know, a new feature of the present discussion. It is brought to light by detining, from the outset, these conceptions with reference to collections of unrestricted multitude. 
$y$ be taken as the 0 of the ordinary algebra of logic. Let $\bar{y}$ be taken as the 1 of that algebra (otherwise symbolized, by some, as $\infty$ ). Let the relation $p<_{y} q$ be regarded as the usual relation of logical antecedent and consequent; and let the subscript of the symbol $<_{y}$ be dropped. by virtue of that usual convention which regards the reference to 0 , not as reference to an arbitrary origin, but as such that $a<b$ has an invariant or absolute sense. So regarded, the system $\Sigma$ possesses an element, 0 , such that whatever element $x$ be chosen, $0<x$; and also an element, 1 , such that whatever element $z$ be chosen, $z<1$. The relation $<$ is transitive. If $a<b$ and $b<a$, then $a=b$. If $a<b$, and also $a \neq b$, the relation $<$ is asymmetrical. Elements such as $p$ and $s$ may first be viewed as determined by some given pair of elements, e. g., by the pair $(a, b)$. The element $p$ is then called the product, the element $s$ is called the sum of this pair; and, in the usual symbols, one may write

$$
a b=p ; \quad a+b=s .
$$

The definitions of the operations of logical nultiplication and of logical addition, may assume the form explained in $\mathbf{1 1 4}$ and $\mathbf{1 1 5}$. Obverses will now appear as elements each of which is what is ordinarily called the negative of the other. Since, in fact, by 95 and 112 , the product of an $O$-collection is the origin, and its sum is the obverse of the origin (see also 80,82), the obverse elements $a$ and $\bar{a}$ are such that $a+\bar{a}=1$, while $a \bar{a}=0$. We shall have the known results (easily verifiable on the basis of the foregoing):

$$
\begin{gathered}
a+a=a ; \quad a c=a ; \quad a 0=0 ; \quad a+0=a ; \\
a 1=a ; \quad a+1=1 ; \quad \bar{a}+b=\bar{a} \bar{b} ; \quad \bar{a} b=\bar{a}+\bar{b} ; \\
(a b) c=a(b c) ; \quad a(b+c)=a b+a c ; \quad a+b c=(a+b)(a+c) .
\end{gathered}
$$

Not only are these results predetermined by the foregoing discussion, but we have in fact given to the principles in question a form much more general than the usual form by so stating the principles from the start that they apply to logical operations upon collections possessing any multitude whatever.

It follows then, that the usual algebra of logic applies without restriction to the system $\Sigma$, which is in so far identical with a totality of logical classes, whereof an infinity are mutually non-equivalent, while all are capable of an unrestricted combination by the operations of logical addition and of logical multiplication.

It is worthy of note that, in terms of the ordinary algebra, the conjugate resultants of a given collection may be defined as follows : Let the logical product of a collection $\beta$ of logical elements be $p$. Let $s$ be the sum of $\beta$. Then any element $q$ such that $p<q<s$ is a resultant of $\beta$. If $r$ is a resultant of $\beta$ 
such that $q r=p$, while $q+r=s$, then $q$ and $r$ are conjugate resultants of $\beta$. If $\beta$ is the pair $(a, b)$ represented in the diagram (1), and if $q$ is a resultant of $(a, b)$ so that $q$ includes the common part of $(a, b)$, but does not extend beyond the limits of $a+b$, then, by repeating $(a, b)$ in the diagrain (2), we may indicate, by shading, the portion of the repeated diagram where $r$ lies, and

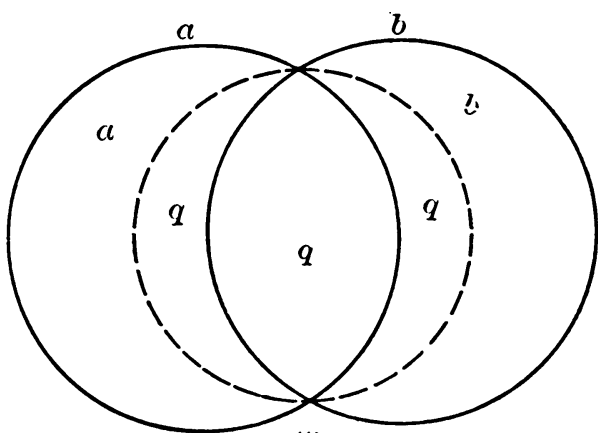

(1)

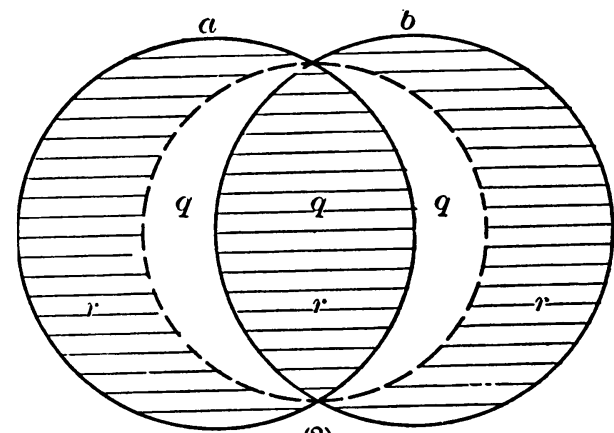

(2)

so the extent of $r$, the conjugate resultant of $q$, in the pair $(a, b)$. It will be observed that $q$ and $r$ have the product $a b$ in common, but supplement each other as to the remainder of $a+b$. If $q$ expands so as to coincide with the whole of $a+b, r$ shrinks to $a b$, and conversely.

The negatives $\bar{q}$ and $\bar{r}$ are the elements whose existence is directly asserted in principle VI.

\section{Chapter VI. The system $\Sigma$ as a generalized space-form.}

119. The inquiry of the previous chapter was primarily devoted to determining what elements exist in $\Sigma$, and how they are arranged. As an incident to this research, the relations of our system $\Sigma$ to the system defined in the algebra of logic was developed. But the consequences of principles V and VI, in their combination, have still other aspects. In particular, the properties of the system $\Sigma$ to which we have already called attention, make its array analogous to that of the points of space. This we are next to see.

120. By definition, all of the elements of any collection are resultants of that collection. But if a collection $\beta$ contains at least one pair of non-equivalent elements, and if $\beta$ at the same time comprises oniy a finite collection of the resultants of some one of those pairs of non-equivalent elements which $\beta$ contains, then there exists an infinite collection of resultants of $\beta$ such that these resultants are not themselves elements of $\beta$. This appears from 89, in combination with principle V. If $\beta$ is an $O$-collection, all the elements of $\Sigma$ are resultants of $\beta$, and are also complements of $\beta$. If $\beta$ is not an $O$-collection, $\Sigma$ contains an infinity of elements which are not resultants of $\beta$. For the 
obverses of the resultants of $\beta$ can none of them be resultants of $\beta$; since if $F(\beta \mid x)$ and $F(\beta \mid \bar{x})$ are both of them true, $O(\beta)$. The obverses of the resultants of $\beta$ are, by definition, complements of $\beta$. But if $\beta$ is not an $O$-collection, $\Sigma$ also contains an infinity of elements which are neither complements nor resultants of $\beta$. For let $(a, b)$ be any pair of conjugate resultants of $\beta$, so that $J(a b ; \beta)$. Since $\beta$ is not an $O$-collection, $E(a b)$ is true (by 95). Every resultant of $\beta$ is a mediator of the pair $(a, b)$. Since $E(a b), a \neq \bar{b}$ and $b \neq \bar{a}$. No element $x$ such that $F(x \mid \bar{a} b)$ is true, and no element $x^{\prime}$, such that $F\left(x^{\prime} \mid a \bar{b}\right)$ is true, can be a resultant of $\beta$ unless $x=b$ or $x^{\prime}=a$. Since the complements of $\beta$, being obverses of the resultants of $\beta$, are mediators of the pair $(\bar{a}, \bar{b})$ we can thus define an infinity of elements which are neither complements of ' $\beta$ nor resultants of $\beta$. All elements, $x$, such that $x \neq \bar{\imath}, x \neq b$ and $F(x \mid \bar{a} b)$; and all elements $x^{\prime}$, such that $x^{\prime} \neq a, x^{\prime} \neq \bar{b}$ and $F\left(x^{\prime} \mid a \bar{b}\right)$, are, namely, neither complements nor resultants of $\beta$. An analogous assertion holds for any other of the pairs of conjugate resultants of the col lection $\beta$. Whatever pair of elements $(a, b)$ we may choose, an infinity of pairs of elements of the form $(a, \bar{b}),(b, \bar{a})(c, \bar{d}),(d, \bar{c})(e, \bar{f}),(f, \bar{e})$, etc., are thereby determined, such that each pair consists of some resultant $c, d, e$ of the pair $(a, b)$, while the other member of each pair is the obverse of the corresponding conjugate resultant of $(a, b)$, No mediator of any one of the pairs $(a, \bar{b}),(c, \bar{d}),(\bar{e}, f)$, etc. (except $a$, or $c$, or $f$, as the case may be), is a resultant of the pair $(a, b)$.

If $a, b, c, d, e, f$, are mutually non-equivalent elements, while the pairs $(a, b),(c, d),(e, f)$, are each of them pairs of conjugate resultants of the same pair, or of the same collection $\beta$, then no resultant of $(\bar{a}, b)$, or of $(a, \bar{b})$, or of $(c, \bar{d})$, or of $(\bar{c}, d)$, or of $(e, \bar{f})$, can be equivalent to any resultant of the other pairs thus defined. If $x$, for instance, is a mediator of $(c, \bar{d})$, so that $F(x \mid c \bar{l}), F(d x \mid c)$, then, with respect to $x$, taken as a base, the conjugate limits of $(a, b)$, or of the collection $\beta$, in question, are, respectively $c$ and $d$; $c$ being the inferior and $d$ the superior limit with respect to the base $x$, of the collection $\beta$. If therefore $x^{\prime}$ is such that $F^{\prime}\left(x^{\prime} \mid e \bar{f}\right)$, $x^{\prime}$ can be equivalent to $x$ only in case each of these elements $x$ and $x^{\prime}$, taken as base, determines, in the manner shown in 100,101 , the same pair of conjugate limits of $\beta$; in which case $c=e, d=f$. For if $F^{\prime}(d x \mid c)$, and $F\left(x f^{\prime} \mid e\right)$, where $(c, d)$, and $(e, f)$ are conjugate pairs, this result follows.

121. If we begin afresh, with a pair $(a, b)$, and then choose a base, $y$, such that $F(y \mid a \bar{b})$, it is thus plain that, for this base $y, a$ is the inferior, and $b$ is the superior limit of the collection of the resultants of $(a, b)$, and that this choice of inferior and superior limits for $(a, b)$, remains invariant for any base that is a mediator of $(a, \bar{b})$, while, if the base is changed to some other pair $(c, \bar{d})(d, \bar{c})$, etc. - $(c, d)$ being a pair of conjugate resultants - the inferior 
and superior limits of $(a, b)$ (the products and sums of the ordinary algebra of logic) vary accordingly. The totality of the expressions employed in the ordinary algebra of logic to represent the relations of a system of classes, will remain invariant as to certain values, and undergoes, for other values, perfectly definite transformations, in case the base with reference to which products and sums are reckoned is altered, so that some class $y$ takes the place which has been assigned to the zero of the ordinary algebra. If the base is, for instance, changed from some element $y^{\prime}$, such that, $F\left(y^{\prime} \mid a \bar{b}\right)$, to some element $y$, such that, $F(y \mid \bar{a} b)$, the product of the pair $(a, b)$ is transformed into what was formerly its sum, and the sum into what was formerly the product. If the new base is an element $y$ such that $F(y \mid \bar{a} \bar{b})$, the product and sum of $(a, b)$ are transformed into a new pair of the conjugate resultants of $(a, b)$. These transformations, somewhat analogous, for the algebra of logic, to a transformation of coördinates in a space system, lead to results which are predetermined by the $F$-relations of the elements of the system $\Sigma$. However the base may be changed, the product of any $O$-collection will be equivalent to the new base; the sum of an $O$-collection will be equivalent to the obverse of the base; and so on.

Meanwhile, any pair $(a, b)$, such that $E(a b)$, while $a \neq b$, forms a means of an exhaustive classification of the elements of $\Sigma$. Given, namely, any element of $\Sigma$, say $x$, there is some determinate pair of resultants of $(a, l)$, say the pair $(k, l)$, such $F(x \mid k \bar{l})$. To the resultants of the pair $(k, \bar{l})$ belongs one resultant, $k$ of $(a, b)$, and there are also an infinite number of possible bases, for which $k$ is product and $l \mathrm{sum}$, of $(a, b)$. No element of $\Sigma$ belongs at once to two of the distinct classes thus defined by selecting pairs of conjugate resultants $(i, j),(k, l)$, such that $i \neq k, i \neq l$, etc., and by then defining the class of the resultants of $(k, \bar{l})$ and of $(\bar{k}, l)$.

Or, again, one may express our present result by saying that if an element $x$ is not a resultant of the pair $(a, b)$, then there exists one and only one pair of conjugate resultants of $(a, b)$, namely the pair $(k, l)$, such that, if the obverse of a determinate one, say $k$, of these two conjugate resultants of $(a, b)$ be chosen, $F(x \mid \bar{k}, l)$, while $x \neq l$.

122. If a pair of elements $(a, b)$ be chosen such that $a \neq b$, and $E(a b)$, it is always possible to find a pair of resultants, $(q, r)$ of $(a, b)$, such that $q$ and $r$ are not mutually conjugate resultants of the pair $(a, b)$, while $q \neq r$, and $E(q r)$, and while $F(a \mid q r)$ and $F(b \mid q r)$ are both of them false. In order to construct such a pair, it is only necessary to choose any resultant $q$, of the pair $(a, b)$, such that $q \neq a, q \neq b$, and then to determine $r$ such that $r \neq q, r \neq b$, and $F(r \mid q b)$. In this case, since $F(q \mid a b)$, and $F(r \mid q b)$, it is impossible that $F\left(a \mid q^{r}\right)$. For if $F(a \mid q r)$ and $F(r \mid q b)$, it follows that $F\left(a \mid q^{b}\right)$; while since, at the same time $F(q \mid a b)$, there results $q=a$, contrary to the hypothesis. Moreover, if $F(b \mid q r)$, and $F(r \mid q b)$, it follows that 
$r=b$; and this again is counter to the hypothesis. Hence $q$ and $r$ are both of them resultants of $(a, b)$, but neither $a$ nor $b$ is a resultant of $(q, r)$.

Consider, now, the collection $\rho$ of all the resultants of the pair $(a, b)$, and the collection $\rho_{1}$ of the resultants of the pair $(q, r)$. Every element of $\rho_{1}$ belongs to $\rho$. But there exists an infinity of elements of $\rho$ such that no one of them either belongs to $\rho_{1}$ or is equivalent to any of the elements of $\rho_{1}$. Since the pair $(q, r)$ is again a pair of non-equivalent elements which is not an $O$-pair, it is possible to determine new pairs $(s, t),(u, v),(w, x)$, etc., without limit, such that the resultants of these pairs form a series, or chain, of collections, $\rho, \rho_{1}, \rho_{2}$, whereof each collection is wholly inclusive of all the elements of each later collection, while each collection contains an infinity of elements that are not included in the later collections, and that are equivalent to none of the elements so included.

It is, in the reverse direction, possible to include any collection $\rho$ of the resultants of a given pair (so long as this is not an $O$-pair), in some more inclusive collection, $\rho^{\prime}$, which then may be enlarged, if necessary, to the collection of the resultants of some new pair, by considering any of the pairs of conjugate resultants of $\rho^{\prime}$.

123. The structure of $\Sigma$ is, therefore, such as to permit this endless determining of internally complete systems of resultants within systems, every such collection comprising an infinite set of elements. This being the case, the question arises whether there is also any sense in which the system $\Sigma$ may be said to possess a "dimensionality" resembling that of space. The answer is that such a conception, in the system $\Sigma$, is capable of arbitrary definition in an infinite number of ways. And such a way, in fact, is suggested by the relation of any inclusive system $\rho$ of the resultants of a pair $(a, b)$, and any included system such as the collection $\rho_{1}$ of resultants of the pair $(q, r)$ defined above.

Suppose, namely, that we arbitrarily define the collection of the resultants of the pair $(q, r)$ as a one-dimensional collection, simply because the totality of these resultants is determined by the naming of the single pair of elements $(q, r)$. In precisely the same sense, it would appear that the resultants of $(a, b)$ or any other pair might be regarded as also of one dimension. But if we consider more carefully, it is plain that the following reason appears for a distinction between the systems $\rho$ and $\rho_{1}$. Let $m$ be any resultant of $(q, r)$, such that $F(q r \mid m)$ while $m \neq q, m \neq r$. In $\rho$, that is, with respect to $(a, b), m$ possesses a conjugate resultant $n$, such that $J[m n ;(a, b)]$, i. e., $J(m n ; \rho)$. Now it is plain that $F(q r \mid n)$ is false. For if $F(q r \mid n)$ and $F(q r \mid m)$ were both at once true, we should have, by 73, every element of $\rho$ a mediator of $(q, r)$, and so $(q, r)$ would be a pair of conjugate resultants of $(a, b)$, which is contrary to the construction as stated in 122. Consider the triad $(q, r, n)$. Since $F(q r \mid m)$, any resultant of the pair $(m, n)$ is a resultant of $(q, r, n)$, 
as can readily be shown by the usual elimination-process. Hence any element of $\rho$ is a resultant of the triad $(q, r, n)$. Hence the triad $(q, r, n)$ possesses resultants which are not resultants of any of the pairs $(q, v),(r, n),(q, n)$. The triad $(q, r, n)$ resembles then a triangle, or two-dimensional complex, when viewed with reference to the pairs $(q, r),(r, n),(q, n)$. Thus $\rho$ can be viewed as a two-dimensional complex in relation to $\rho_{1}$. An analogous result holds whatever pair $(a, q),(b, v)$, etc., we choose from the resultants of $\rho$, so long as the resultants of this selected pair form only a portion of the resultants of $\rho$, while elements equivalent to none of the resultants of the selected pair belong to $\rho$.

But we are not limited in our selection to the whole system $\rho$, in order to be able to define such triads. Consider next the triad $(q, b, n)$. By 122, $q$ and $r$ have been so defined that $F(r \mid q b)$. Since, by construction, $F(m \mid q r)$, it follows that $F(m \mid q b)$. Were $n$ also such that $F(n \mid q b)$, every resultant of $(a, b)$ would be also a resultant of $(q, b)$, which is false by construction. Hence $F\left(n \mid q^{b}\right)$ is false. Were $F(q \mid b n)$ true, then since, as just shown, $F(m \mid q b)$, we should have true $F(m \mid b n)$, and hence, since $F(b \mid m n)$, it would follow that $b=m$, which is impossible by construction. For $F(m \mid q r)$, while $m \neq q$, and $m \neq r$. Finally, if $F(b \mid q n)$ were true, then, since $F(m \mid q b)$, it follows that $F(m \mid q n)$, and hence, since $F(q \mid m n)$, it would follow that $q=m$, which is again false by construction.

Therefore, no one of the elements of the triad $(q, b, n)$ is a mediator of the other pair. The conjugate resultant, in this triad, of the element $n$, is an element which is a mediator of the pair $(q, b)$ (by 98); and hence, since, $n$, a mediator of $(q, r)$, is the conjugate resultant of $n$ in $\rho$, it is impossible that the resultants of the triad $(q, b, n)$ exhaust the collection $\beta$. Meanwhile, the triad $(q, b, n)$ possesses resultants which are not resultants of any one of the pairs $(q, b),(b, n),(q, n)$. And so the triad $(q, b, n)$ may be viewed as a twodimensional complex.

It thus follows both that the resultants of $\rho$, taken as a whole, can be viewed as the resultants of a triad, if we choose, rather than as the resultants of a pair; and that triads such as $(q, b, n)$ can be defined, in $\rho$, in such wise that a triad $(q, b, n)$ possesses resultants which are not resultants of any of its single pairs, and which are still but a part of the resultants of the system $\rho$. Any such triad, however, may be viewed as a two-dimensional structure.

The viewing of $\rho$ as a two-dimensional complex with reference to $\rho_{1}$ as a onedimensional complex, is typical of a process which can be repeated any number of times. For, since $\rho_{1}$ is itself inclusive of $\rho_{2}$, etc., $\rho_{1}$ may be viewed, with reference to these included systems, as a complex possessing two, three, or $n$ dimensions, where $n$ is any whole number. According as this is done, $\rho$ comes to be viewed, with reference to a particular series of included collections, as of three, four, or $n+1$ dimensions. 
The result of the foregoing considerations is that, within any portion of the system $\Sigma$ which contains at least one pair of non-equivalent elements, we can define, pairs, triads, etc., in brief, collections of any finite number of mutually non-equivalent elements, such that, if such a collection, say $\alpha$, possesses $n$ elements, there exist resultants of the whole collection which are not resultants of any partial collection of the elements, containing only $n-1$, or $n-2$, or any less number of these elements themselves.

We may call the complexes of the resultants of such collections $n$-dimensional complexes. But it is observable that any such complex, once given, may also be treated, by the proper choice of conjugate resultants, as a complex of the resultants of a single pair, and so as a one dimensional complex. So that all such dimensionality is entirely relative to processes and structures of the type that we have just been defining.

124. Such structures become, however, of a more positive significance if we take account of the following application.

By a line shall be meant a structure of the general type of the chains of 83 , only completed by the insertion of certain mediators. A line shall be a collection of elements such that in case of any triad of the elements of the collection, one member of this triad is the resultant of the pair composed of the other two.

And, in particular, the lines that we are here first and mainly to consider are to be subjected to the entirely arbitrary restriction (foreign to the first principles of our system $\Sigma$, but quite capable of being satisfied by a due selection of its elements as their existence has now been established), that if any two nonequivalent entities of a line are given, no other line, in the set of lines that we are to consider shall at once contain both of these elements. ${ }^{*}$ In other expression, let the collections which are to be called lines be so selected that, if $(a, b, c)$ is any triad of elements belonging to the same line, $F(a b c)$ is true; while, if $F(a p q)$ is true and $F(b p q)$ is true, and if at the same time $F(a b p)$ is false, then we shall so select that $p=q$; so that if $(a, p, q)$ is a triad of elements belonging to one of the lines now to be selected, while $(b, p, q)$ is a triad belonging to another of these lines, and while $(a, b, p)$ is no linear triad at all, then we shall be required so to select that $p=q$.

125. That selections of this sort are possible the theory of the chains, as developed in 83 sqq., has already shown. Such chains as were there defined. might be constructed, as we now may observe, intersecting one another any number of times. For if $(c, d)$ be any pair of elements belonging to a chain, the resultants of $(c, d)$ form no single chain, but lie in sets subject to principle VI, which demands the existence of conjugate resultants, not only in the collection of the resultants of the pair $(c, d)$ itself, but in every one of the countless collections of resultants of the pairs intermediate between $c$ and $d$, as

* The development is here wholly due to KEMPE's initiative. 
these pairs have been characterized, in their mutual relations, in 122, 123 . It is possible, within the limits of any pair of non-equivalent elements $(c, d)$, to define any number of segments, that is of intermediate pairs $\left(p_{1}, p_{2}\right),\left(p_{2}, p_{3}\right)$, $\left(p_{3}, p_{4}\right)$, etc., each of which consists of mediators of $(c, d)$, while all the elements concerned form triads such that $F\left(p_{i} p_{j} p_{k}\right)$ is true of any one of these triads. A chain, or rather a series of chains, can be run through such a series of intermediate pairs, according to any desired principle of selection from amongst the elements present in the various systems of resultants encountered. By virtue of the results stated in 117 , a set of successive chains can be enlarged to a complete line, resembling perfectly, in its structure, a continuous geometrical line, by a mere insertion of intermediates and sets of intermediates. The special principle of selection assigned for the lines now to be considered will not only ensure that two lines have never more than one intersection, but in combination with the definition of a line will also exclude that degree of wealth of elements which forbids the arrangement of all the resultants of any pair of elements in $\Sigma$ in a single linear serial order. For in the system $\Sigma$ as a whole, if $m$ and $n$ are equivalent neither to $a$ nor to $b$ and are conjugate resultants of $(a, b), F(m \mid a b)$ and $F(n \mid a b)$ are both true; while $F(m \mid n b)$ and $F(n \mid m b)$ are both false. The principle laid down for the selection of line-collections will therefore forbid the inclusion in a given line of more than a single pair of conjugate resultants of any one pair. Thus, if $c$ and $d$ belong to the line, $c$ and $d$ themselves will be conjugate resultants of their own pair. And the intermediate elements of the line will be in $\Sigma$ resultants of that pair. But no conjugate in $(c, d)$ of any such resultant of $(c, d)$ as belongs to the line, will lie in the line, except $c$ and $d$ themselves.

126. Since collections possessing the dimensional structure described in $\mathbf{1 2 3}$ exist in any region of $\Sigma$, it will alvays be possible to define systems of lines as follows : Let $(d, e, f)$ be any triad such that it possesses resultants not contained amongst the resultants of $(d, e),(e, f),(d, f)$. If $(d, e),(e, f)$, $(d, f)$, belong to lines that are amongst those lines which are here in question, and if $x$ be any resultant of $(d, e, f)$ which is not a resultant of $(d, e)$, nor yet of $(e, f)$, nor yet of $(d, f)$, then it will be possible to regard $(x, d)$ or $(x, e)$, or $x$ united with any element of the lines $(d, e)$, etc., as constituting a new set of segments of lines. The result will be a two dimensional complex of elements. That method of construction of the $n$-dimensional collections or complexes of elements of $\Sigma$ which has been indicated, enables us to regard these complexes, with all the lines, segments, etc., which are involved, as possessing an extent and variety of elements such as to permit us to define new sets of elements beyond any segments or bounded complexes once defined. Therefore, in selecting elements for our present purpose, we may regard these new elements as extensions of the lines and other complexes, while the dimensionality of the 
complexes of lines which we nay thus consider is subject altogether to our pleasure, under the conditions now in general laid down.

If, in consequence of the foregoing considerations, we compare the set of relations that we can thus define with the relations known to geometry, a natural method presents itself in the form of a juncture that may now readily be effected between our account and Dr. Veblen's System of Axioms for Geometry, (Transactions of the American Mathematical Society, July, 1904).

Dr. Veblen's expression "in the order $A B C$," becomes, in our terms, the assertion $F(b \mid a c)$. If we agree, in studying the constitution of our system of lines, to take explicit account only of non-equivalent elements, if we here call our elements points, and if we also adopt Dr. Vebles's definitions, his axioms appear in our statement as follows :

Dr. VerLen's first axiom covers our own principles III and IV, according to which our system contains a pair of elements. Axiom II of Dr. Veblen's set, interpreted in our terms, declares that if $F(b \mid a c), F(b \mid c a)$. This needs for us, no comment. Axiom III asserts that, if $F(b \mid a c)$, then $F(c \mid b a)$ is false. Our own principles require that, in this case, $b=c$; and the axiom may therefore be regarded simply as excluding us from treating certain pairs of equivalent elements as distinct elements. This is merely a principle of selection. Axiom IV asserts that if $F(b \mid a c)$, then $a \neq c$. Our principles like Kempe's, require that if $a=c, a=b=c$. Axiom IV, therefore, again excludes the regarding of certain equivalent elements as, for the present purpose, distinct. Axiom V of Dr. Veblen's set requires that if $a \neq b, c$ exists such that $F(b \mid a c)$. This principle is provided for by our principles, which show that every pair defined in any of our sets of lines may be viewed as included in larger systems possessing linear $F$-relations. Axiom VI defines the important, but for us, quite arbitrary principle that governs the selection of the line-elements : "If points $c, d(c \neq d)$ lie on the line $(a, b)$, then $a$ lies on the line $(c, d)$." This agrees with our foregoing statement in 124. Only, with us, this is merely a principle of selection. Axioms VII, IX, relate to dimensionality, and demand points existent in triads and tetrads such as we have provided for in the foregoing. For us, such requirements are permitted by the system $\Sigma$ in an infinity of ways.

Axiom VIII, the "triangle transversal" axiom runs, in our terms, thus: If the triad $(a, b, c)$ is of the two-dimensional character described in 123, and if $d$ and $e$ exist such that $F(e \mid a c)$ and $F(c \mid b d)$, then $f$ exists such that $F(f \mid a b)$, and $F(f e d)$.

This axiom for us is, if we grant a certain mode of selection of elements, a theorem, resulting from the theorem of 99 - an incidental result, as it may be called, of the theory of conjugate resultants. That is, theorem 99 secures the existence of elements which may be selected so as to verify axiom VIII. 
The theorem of 99 runs that if $F(\beta \mid \alpha), \beta$ and $\alpha$ have at least one resultant in common.

By the hypothesis of axiom VIII, $F(c \mid e \bar{u})$ and $F(c \mid b c l)$. Hence $F\left(e \bar{a} \mid b c^{d}\right)$. Hence follows $F_{(}(e \bar{l} \mid b a)$. By $99,(e, \bar{d})$ and $(b, a)$ have in the system $\Sigma$ at least one resultant in common (they have in fact, in $\Sigma$ an infinite number in common). Call this resultant $f^{*}$. Then $f^{\prime}$ exists such that $F\left(f^{\prime} \mid a b\right)$, and $F(f \mid e \bar{l})$; i. e., $F(e \mid . f l)$ so that (at least) $F(f e d) .{ }^{*}$ That the common resultants here in question should belong as points to the system of lines that we have selected from the system $\Sigma$, is itself a matter of the mode of selection used. The properties of the system $\Sigma$ simply insure the possibility of such a selection.

Axiom XI, Dr. Veblen's form of the postulate of continuity, is provided for by our own result, holding for the system $\Sigma$ in general, stated in 117. This result ensures the possibility of the continuity of the line-collections, in case we choose to select suitable sets, precisely as the same result ensures in the system $\Sigma$ as a whole, the existence of "products" and "sums." Axiom X, which limits Dr. Veblen's system to three dimensions, is for us a perfectly possible, but again quite arbitrary limitation; and the same can be said of the parallel line axion XII, which concerns wholly the limitation of the selection of the lines admitted into a given system.

Our own "transversal" theorem, in 99, justifies, in terms of our principles, the remark made by Mr. KEMPE, upon the basis of his postulates, to the effect that any $F$-collection which contains a finite number $n$ of elements that belong to the sets selected as the lines of the foregoing discussion, represents a definite configuration of points in a space of $n-2$ dimensions.

Thus $F(a b c)$ implies that $(a, b, c)$ is a triad of points on one line. $F(a b \mid c d)$ is to be interpreted as follows: The pairs $(a, b)$ and $(c, d)$, lie by hypothesis, upon some selected pair of lines of our geometrical set. The problem is, how are these two lines to be related? The assertion $F(a b \mid c d)$ requires, by 99 , that, in $\Sigma, x$ should exist such that $F(a b \mid x)$ and $F(c d \mid x)$. If then $x$ be viewed as one of the selected elements of the geometrical set in question, the assertion $F(a b \mid c d)$ may be viewed as the assertion that the lines through the segments $(a, b)$ and $(c, d)$ have in common a point of intersection which belongs to the mediators of $(a, b)$ i. e., to the points of the segment $(a, b)$, and also to the segment $(c, d)$. On the other hand, if $F(a b \mid x)$ and $F(c d \mid x)$ are given, our principles require that $F(a b \mid c d)$. So that this form of assertion defines a pair of intersecting lines. The assertion $F(x \mid a b c)$ defines a resultant of the triad $(a, b, c)$. If this triad is to be viewed, in the way heretofore defined, as determining a complex of two dimensions, then $x$ is a point

* KEMPE's theory is explicitly based upon two forms of the transversal theorem, assumed at the outset. For this our statement of the theory substitutes the postulated existence of conjugate pairs. What KEMPE sets at the beginning we thus reach at the end. 
lying within the triangle $(a, b, c)$. If $F\left(a b c \mid d e f^{\prime}\right)$, wherein all the elements are mutually non-equivalent, and wherein each triad is to be viewed as a twodimensional complex, then $x$ exists common to these two areas, or two-dimensional complexes here in question. Thus all the intersection theorems of geometry may be stated in the form of the assertion of $F$-relations, with a due regard to the limitations of the classes of selected elements.

The principle of continuity is, for such a geometry, merely a principle of the selection of the elements, a principle which the system $\Sigma$ permits, but does not require to be carried out.

Instead of such systems of lines as have here been selected, systems of lines whereof any two have two, three or $n$ intersections, are perfectly permissible, so far as the system $\Sigma$ is concerned. The possibility of a free, but definite variation of space-forms in a infinite number of ways, is thus provided for by the system $\Sigma$; and the outlook for a basis for generalized space-conceptions is all the more attractive, since the structure of the system $\Sigma$, based as it is upon fundamental logical principles, makes a test of the logical possibility of any proposed geometry a perfectly definite task - namely the task of seeing whether $\Sigma$ actually contains complexes which are suitable to embody the desired space-form.

Since $O$-collections at once possess, as their resultants, all of the elements of $\Sigma$ at once, no definite view of their dimensional structure is any longer possible. Hence selections suitable for space-forms must exclude $O$-collections; and so, as KEMPE again points out, no geometrical set contains the obverse of any of the elements of the set. It follows that "spaces," defined in the foregoing way, always occur in $\Sigma$ in pairs, such that to any one space-form $\sigma$ there always corresponds a space-form, or collection $\bar{\sigma}$, constituted of the obverses of the elements of $\sigma$. These two space-forms are related, in KeMPE's view, somewhat as two hemispheres.

Finally, since metrical relations can be reduced, in the known way, to ordinal relations, KeMPE has briefly pointed out (as mentioned in the introduction to this paper), that sets of the elements of $\Sigma$ can be so selected that operations corresponding to the addition and multiplication of the ordinary algebra of quantity, will enable us to select elements that may be viewed (with reference to certain arbitrarily assumed constant triads of reference-elements, i. e., bases), as the sums or as the products of given pairs of elements. Hence, without introducing new elements, the elements of $\Sigma$, if viewed in certain ways, enable us to define, not only the algebra of logic, but the algebra of quantity.

Note on the independence of the six principles.

That principle $\mathrm{V}$ is independent of the other principles is proved, in 84 , by the assumption of a system $\Sigma^{\prime}$ consisting of a single pair of obverses. 
That principle IV is independent is proved by the reasoning used in 35 . For if we assume a system $\Sigma^{\prime \prime}$, all of whose monads and possible colleotions are to be defined as $O$-collections, while the system itself comprises any arbitrarily chosen number of elements, all the principles except IV and V are satisfied by the possibly existent collections of $\Sigma$ ", while principle $V$ is satisfied " vacuously," since no pair of non-equivalent elements exist. But in such a system principle IV is false ; since all the elements are, by the definition of equivalence. mutually equivalent.

Principle III, and that principle alone, would be violated by an empty system; and that principle is therefore independent.

If, instead of the $O$-collections, we had used, as the basis of our account of the system $\Sigma$, the indeterminate $F$-collections of $\Sigma$, all the principles I, III, IV, $\mathrm{V}$, VI would remain true if we viewed them as statements regarding indeterninate $F$-collections, and could therefore have been used as principles for the system of $F$-collections. But principle II is false if interpreted as applying to $F$-collections. For $F(x x)$ is always true, since $O(x \bar{x})$. But from $F(\eta x)$ does not follow $F(\eta)$. Hence principle II is independent of the other principles.

If we consider the class of those $O$-collections of the system $\Sigma$ which are either pairs or triads, but which contain no greater number of elements than three, we may call this class the class of the $O_{1}$-collections. For this class of collections, principle $I$ is false, since the $O_{1}$-triads cannot be enlarged, by the adjunction of any new members. In order to apply prineiple II, the hypothesis of that principle must be read as applying to a collection $\beta$ which, in order that it should be an $O_{1}$ collection at all, must not exceed a triad. If $O_{1}(\beta)$, where $\beta$ is a pair or a triad (so that $O(\beta)$ is also true), and if $O_{1}\left(\delta b_{n}\right)$ is true, where $b_{n}$ is any one of the two (or three) elements of $\beta$, then $\delta$ itself (by the definition of the $O_{1}$-collections), cannot exceed a pair ; otherwise $\left(\delta, b_{n}\right)$ would be a collection of more than three elements. Principle II then becomes equivalent to the assertion that, if $\beta$ is a pair or a triad, and if $\delta$ is a monad or a pair, if $O(\beta)$ is true, and if $O\left(\delta b_{n}\right)$ is true, then $O(\delta)$ is true. Hence principle II is true of the $O_{1}$-collections. Principles III and IV are obviously true of the elements of $\Sigma$, considered with reference to the $O_{1}$-collections. The equivalences and nonequivalences are, in fact (because of what is proved in 27-30), unchanged by the limitation of our view to the set of $O_{1}$-collections, since all equivalences and nonequivalences are already concerned in determining the relations of obverses. And principles $\mathrm{V}$ and $\mathrm{VI}$, which require the existence of certain $O$-triads (such as are also $O_{1}$-collections), remain true, although the hypothesis of principle VI becomes limited, in its application, to the mention of the complements of pairs and of monads. Thus principle $I$ is independent of the other principles.

To prove the independence of principle VI : 
Consider two pairs of mutually obverse collections of elements of $\Sigma$, viz., $\alpha$ and $\bar{\alpha}$, such that each is a line of elements, defined as follows :

(1) Every element $a_{n}$ of $\alpha$ is a mediator of a pair $(x, y)$ of elements of $\Sigma$, such that neither $x$ nor $y$ belongs to $\alpha$, while $E(x y)$ is true. Thus $F\left(a_{11} \mid x y\right)$ is true of every element $a_{n}$ of $\alpha$.

(2) Whatever triad of elements of $\alpha$ be chosen, say the triad $\left(a_{m}, a_{n}, a_{r}\right)$, $F\left(a_{m} a_{n} a_{r}\right)$ is true of this triad.

(3) Whatever element $a_{n}$ of $\alpha$ is chosen, $a_{q}$ and $a_{r}$ exist, belonging to the collection $\alpha$, and such that $F\left(a_{q} \mid x a_{n}\right)$ and $F\left(a_{r} \mid y a_{n}\right)$, while $a_{q} \neq a_{n}$, and $a_{r} \neq a_{n}$.

(4) No two distinct elements of $\alpha$ are mutually equivalent elements of $\Sigma$.

(5) Whatever pair $\left(a_{q}, a_{r}\right)$ be chosen from amongst the elements of $\alpha, a_{m}$ exists such that $F\left(a_{m} \mid a_{q} a_{r}\right)$ is true.

From this definition of the line $\alpha$, the properties of the obverse collection $\bar{\alpha}$ at once follow. The elements of $\bar{x}$ are mediators of the pair $(\bar{x}, \bar{y})$. No element of $\bar{\alpha}$ can be equivalent to any element of $\alpha$; for if any mediator of $(\bar{x}, y)$ is equivalent to a mediator of $(x, y)$, then $O(x y)$ is true, which opposes condition (1). If then $c$ and $d$ be distinct elements, chosen in any way from the total collection $(\alpha, \bar{\alpha}), c \neq d$ by construction.

Whatever pair $(c, d)$ of elements of $(\alpha, \bar{\alpha})$ be chosen, it follows that there exists $g$, also belonging to $(\alpha, \bar{\alpha})$, and such that $g \neq c, g \neq d$, and $F(g \mid c d)$. For if $c$ and $d$ both belong to $\alpha$, the existence of $g$, as an element of $\alpha$, follows directly from condition (5). If $c$ and $d$ be both chosen from $\bar{\alpha}$, a precisely analogous result holds true. But if $c$ be chosen at random from $\alpha$, and $d$ from $\bar{\alpha}$, then let $c=a_{n}$, and let $d=\bar{a}_{q}$, where $\bar{a}_{q}$ is such that its obverse $a_{q}$ is such that $F\left(a_{q} \mid x a_{n}\right)$. Hereupon, choose in $\alpha$ an element $a_{t}$ such that $F\left(a_{t} \mid a_{t} x\right)$. This, by condition (3), is always possible. Since $F\left(a_{q} \mid x a_{n}\right)$ and $F\left(a_{t} \mid x a_{q}\right)$, it follows that $F\left(a_{t} \mid a_{q} \bar{a}_{n}\right)$ is true; hence $F\left(\bar{a}_{t} \mid \bar{a}_{q} a_{n}\right)$ is true; and thus, if $g=\bar{a}_{t}, F(g \mid c d)$ is true of an element $g$ which belongs to $(\alpha, \bar{\alpha})$. Similarly, were it true that $c=a_{n}$, and $d=\bar{u}_{r}$, where $\bar{u}_{r}$ is an element such that $F\left(a_{r} \mid y a_{n}\right)$, then we might choose $a_{1 c}$ such that $F\left(a_{i c} \mid y a_{r}\right)$. This, by condition $(3)$, is also possible. Eliminating $y$ we have $F\left(a_{n r} \mid a_{r} \bar{a}_{n}\right)$; i. e., $F\left(\bar{a}_{1 c} \mid \bar{a}_{r} a_{n}\right)$; so that, if $g=\bar{a}_{t c}$, we again have $g$ belonging to the total collection $(\alpha, \bar{\alpha})$, and such that $F(g \mid c d)$ is true. Since all pairs of elements $(c, d)$ thus chosen from $(\alpha, \bar{\alpha})$ are, by construction pairs of mutually non-equivalent elements ; since $g$ always exists, belonging to $(\alpha, \bar{\alpha})$ and such that $F(g \mid c d)$ is true; and since $\bar{y}$ also belongs to $(\alpha, \bar{\alpha})$; it follows that the collection $(\alpha, \bar{\alpha})$ contains sufficient elements to satisfy, with respect to any pair of elements of $(\alpha, \bar{x})$, the demands of principle $\mathrm{V}$, without going beyond the elements of this collection $(\alpha, \bar{\alpha})$ itself. That is, whatever pair of elements of $(\alpha, \bar{\alpha})$ be chosen, an element of $(\bar{x}, x)$ exists which is no obverse of either of the elements of the pair, and which forms an $O$-triad when adjoined to the pair. 
Hereupon let us consider a system $\Sigma_{a}$, which shall consist solely of the elements of the collection $(\alpha, \bar{\alpha})$. Let there be formed, of the elements of this system $\Sigma_{a}$, collections which we shall call the $O_{a}$-collections. These $O_{a}$-collections shall be identical with those collections of the elements of $(\alpha, \bar{\alpha})$ which are $O$-collections in $\Sigma$. The $O_{a}$-collections of the system $\Sigma_{a}$ will now conform, by construction, to principles $\mathrm{I}-\mathrm{V}$ of the system $\Sigma$.

But, in case of the $O_{a}$-collections, principle VI will be violated. For (returning to the system $\Sigma$ ) consider any element $a_{n}$ of $\alpha$; and consider the totality of those elements $a_{q}$, belonging to $\alpha$, and such that $F\left(a_{q} \mid x a_{n}\right)$ is true in respect of the system $\Sigma$. Call this totality the collection $\alpha^{\prime}$; so that $\alpha^{\prime}$ is the collection of those elements of $\alpha$ which, in $\Sigma$, are mediators between $a_{n}$ and $x$. By condition (1), $x$ itself does not belong to $\alpha$, and so does not belong to $\alpha^{\prime}$. Now, in the system $\Sigma$, the element $a_{n}$, since it belongs, by construction, to the collection $\alpha^{\prime}$, possesses a conjugate resultant with respect to $\alpha^{\prime}$. Let $r$ be this resultant, so that, in $\Sigma, \nu_{r}\left(a_{n} r ; \alpha^{\prime}\right)$ is true. It is manifest that $r$ is an element such that $F\left(r \mid a_{n} x\right)$. Yet $r$ is no element of the collection $\alpha^{\prime}$ or of the total collection $(\alpha, \bar{\alpha})$. For if $r$ were an element of $\alpha$, then, by conditions (3) and (4), $a_{k}$ would exist, belonging to $\alpha$, and such that $F\left(a_{k} \mid r x\right)$, while $a_{k} \neq r$, and $a_{k} \neq x$. But $a_{k}$ would also belong to $\alpha^{\prime}$, and by the definition of a conjugate resultant $F\left(a_{k} \mid r a_{n}\right)$ would be true. Now $F\left(r \mid a_{n} x\right)$ is true; and from $F\left(a_{k} \mid r x\right)$ and $F\left(r \mid a_{n} x\right)$ follows $F\left(r \mid a_{k i} a_{n}\right)$. If, at the same time, $F\left(a_{k i} \mid r a_{n}\right), a_{k}=r$; which is contrary to the hypothesis. Thus $r$ is no element of $\alpha$, and also cannot be any element of $\bar{\alpha}$. For since $F\left(r \mid a_{n} x\right)$, if there existed $\bar{u}_{l}$ such that $r=\bar{a}_{q}$, $F\left(\bar{a}_{q} \mid a_{n} x\right)$, and hence $F\left(\bar{a}_{q} \mid x y\right)$, would be true of some element $a_{\eta}$ of which $F\left(a_{q} \mid x y\right)$ would also be true. In that case $O(x y)$, which is contrary to condition (1).

Since $r$ does not belong to $\alpha$ nor yet to $\bar{\alpha}, r$ does not exist in the system $\Sigma_{a}$. There is then, in $\Sigma_{a}$, no element capable of meeting the requirements of principle VI as applied, in this system $\Sigma_{a}$, to the partial collection $\alpha^{\prime}$. So principle VI fails in $\Sigma_{a}$; and is therefore independent of the other principles.

HARVARI UNIVERSITY,

CaMbridge, Mass. 